University of Louisville

ThinkIR: The University of Louisville's Institutional Repository

Electronic Theses and Dissertations

$12-2020$

\title{
Imparting 3D representations to artificial intelligence for a full assessment of pressure injuries.
}

\author{
Sofia Zahia \\ University of Louisville
}

Follow this and additional works at: https://ir.library.louisville.edu/etd

Part of the Bioimaging and Biomedical Optics Commons, Biomedical Devices and Instrumentation Commons, and the Other Computer Engineering Commons

\section{Recommended Citation}

Zahia, Sofia, "Imparting 3D representations to artificial intelligence for a full assessment of pressure injuries." (2020). Electronic Theses and Dissertations. Paper 3533.

https://doi.org/10.18297/etd/3533

This Doctoral Dissertation is brought to you for free and open access by ThinkIR: The University of Louisville's Institutional Repository. It has been accepted for inclusion in Electronic Theses and Dissertations by an authorized administrator of ThinkIR: The University of Louisville's Institutional Repository. This title appears here courtesy of the author, who has retained all other copyrights. For more information, please contact thinkir@louisville.edu. 


\title{
IMPARTING 3D REPRESENTATIONS TO ARTIFICIAL INTELLIGENCE FOR A FULL ASSESSMENT OF PRESSURE INJURIES
}

\author{
By \\ Sofia Zahia \\ B.A., Enseirb-Matmeca, France, 2015 \\ M.A., Enseirb-Matmeca, France, 2017

\begin{abstract}
A Dissertation
Submitted to the Faculty of the in Partial Fulfillment of the Requirements

for the Degree of

Doctor of Philosophy

in Computer Science and Engineering

Department of Computer Science and Engineering

University of Louisville

Louisville, Kentucky
\end{abstract} \\ J.B. Speed School of Engineering of the University of Louisville
}

December 2020 



\title{
IMPARTING 3D REPRESENTATIONS TO ARTIFICIAL INTELLIGENCE FOR A FULL ASSESSMENT OF PRESSURE INJURIES
}

\author{
By Sofia Zahia \\ B.A., Enseirb-Matmeca, France, 2015 \\ M.A., Enseirb-Matmeca, France, 2017
}

A Dissertation approved on

December $3^{\text {rd }} 2020$

By the following Dissertation Committee:

Dissertation Director

Dr. Adel Elmaghraby (CSE)

Dissertation Co-Director

Dr. Maria Begonya Garcia Zapirain Soto (External)

Committee Member

Dr. Ibrahim Imam (CSE)

Committee Member

Dr. Hui Zhang (CSE)

Committee Member

Dr. Daniel Sierra Sosa (CSE)

Committee Member

Dr. Monica Gentili (Industrial Eng) 


\title{
DEDICATION
}

This dissertation is dedicated to my parents

\author{
Mr. Abderrahim Zahia \\ and \\ Mrs. Rabia Khettat \\ and my sister
}

Ms. Sara Zahia

who have always showered me with their endless love and valuable educational

opportunities 


\section{ACKNOWLEDGMENTS}

The work of this dissertation could never have been achieved without the support and guidance of many people. I would like firstly to express my gratitude to my advisor in Dr. Adel Elmaghraby for giving me the invaluable opportunity to pursue the $\mathrm{PhD}$ at the University of Louisville. He has always been present and provided me with so many insights, suggestions and kind advice. It is also with a heartfelt appreciation that express my thanks to my disseration co-director Begoña Garcia-Zapirain for her support, motivation and enthusiasm. Their advisory has enabled me to grow as a passionate independent thinker, always aiming to learn and discover new knowledge. I would like to thank my committee members: Dr. Ibrahim Imam, Dr. Hui Zhang, Dr. Daniel Sierrasosa, and Dr. Monica Gentili.

A very special thanks to Marta and Alba who have shared the Ph.D journey with me, and considered me as one them and showered me with their love and kindness. I would also like to thank all my friends who made this journey much more enjoyable, especially my dear and best friend Fadoua, with whom I shared irreplaceable moments and laughters beyond price, when we were roommates in the US and also when I was 4000 miles away.

Lastly and most importantly, I am genuinely thankful to my parents and sister for their unconditional love and support in each and every step I took in my life. Without them I would have never taken shape. I will always seek to be the best version of me and do you proud. 


\begin{abstract}
IMPARTING 3D REPRESENTATIONS TO ARTIFICIAL INTELLIGENCE FOR A FULL ASSESSMENT OF PRESSURE INJURIES
\end{abstract}

Sofia Zahia

December 3, 2020

During recent decades, researches have shown great interest to machine learning techniques in order to extract meaningful information from the large amount of data being collected each day. Especially in the medical field, images play a significant role in the detection of several health issues. Hence, medical image analysis remarkably participates in the diagnosis process and it is considered a suitable environment to interact with the technology of intelligent systems. Deep Learning (DL) has recently captured the interest of researchers as it has proven to be efficient in detecting underlying features in the data and outperformed the classical machine learning methods. The main objective of this dissertation is to prove the efficiency of Deep Learning techniques in tackling one of the important health issues we are facing in our society, through medical imaging. Pressure injuries are a dermatology related health issue associated with increased morbidity and health care costs. Managing pressure injuries appropriately is increasingly important for all 
the professionals in wound care. Using $2 \mathrm{D}$ photographs and $3 \mathrm{D}$ meshes of these wounds, collected from collaborating hospitals, our mission is to create intelligent systems for a full non-intrusive assessment of these wounds. Five main tasks have been achieved in this study: a literature review of wound imaging methods using machine learning techniques, the classification and segmentation of the tissue types inside the pressure injury, the segmentation of these wounds and the design of an end-to-end system which measures all the necessary quantitative information from 3D meshes for an efficient assessment of PIs, and the integration of the assessment imaging techniques in a web-based application. 


\section{TABLE OF CONTENTS}

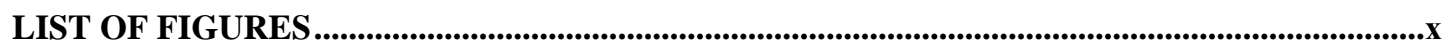

LIST OF TABLES ................................................................................................................................................ $\mathrm{xV}$

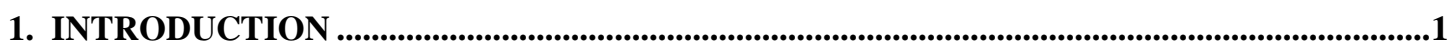

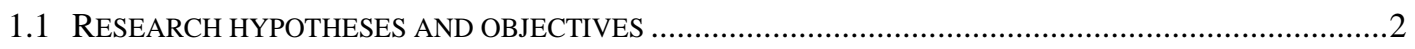

1.2 SCIENTIFIC AND SOCIAL IMPACT AND CONTRIBUTION ..........................................................

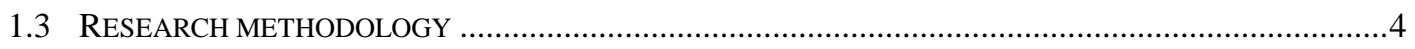

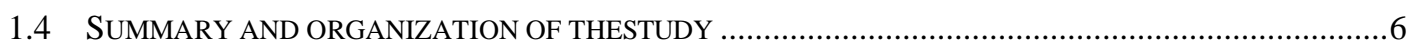

2. LITERATURE REVIEW ..............................................................................................................................8

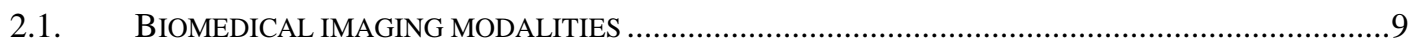

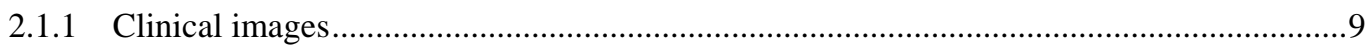

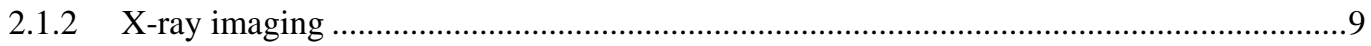

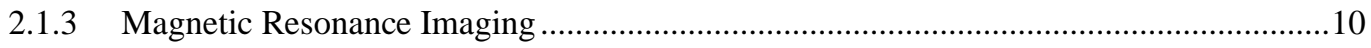

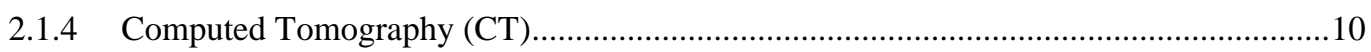

2.1.5 Ultrasound Imaging ..............................................................................................

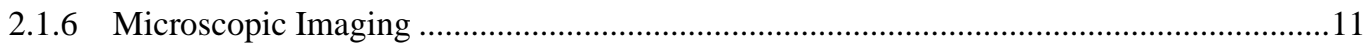

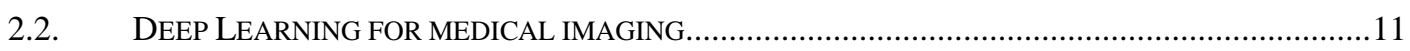

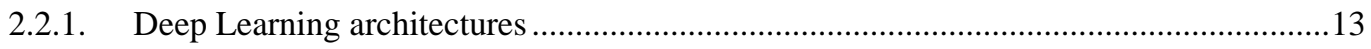

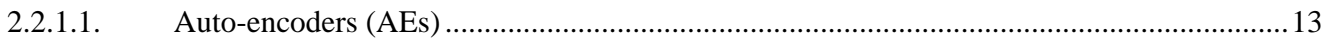

2.2.1.2. 2D Convolutional Neural Networks (2D CNNs) ..................................................... 14

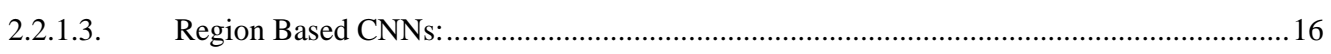

2.2.1.4. 3D Convolutional Neural Networks (3D CNNs) …................................................. 17 
2.2.1.5. Recurrent Neural Networks (RNNs) …............................................................. 18

2.3. PRESSURE INJURY ASSESSMENT ............................................................................ 19

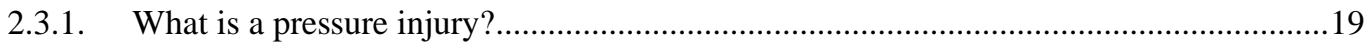

2.3.2. Wound imaging with machine learning ................................................................23

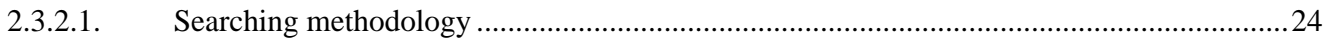

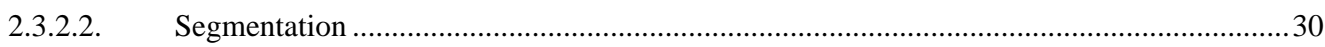

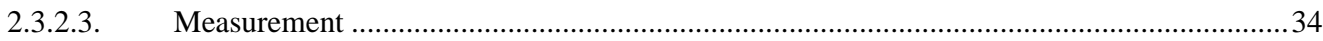

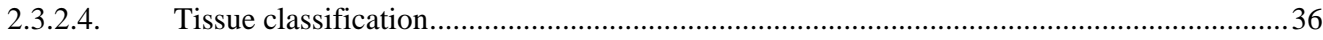

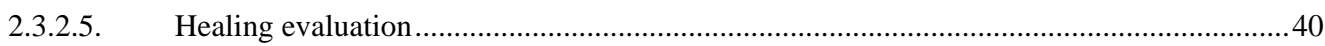

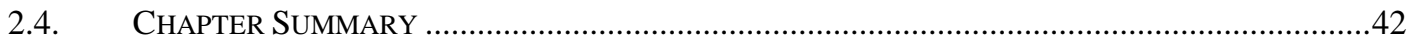

3. PRESSURE INJURY TISSUE CLASSIFICATION ...........................................................43

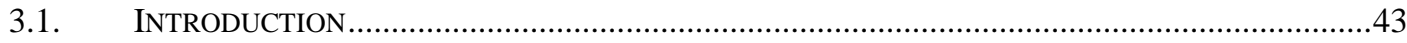

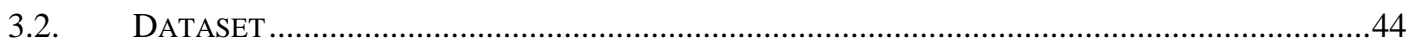

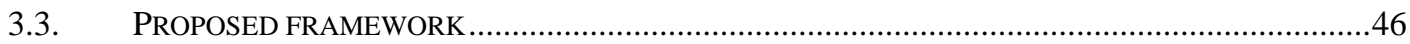

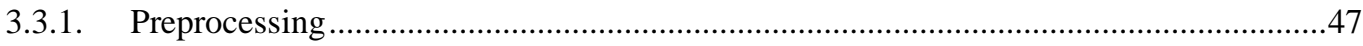

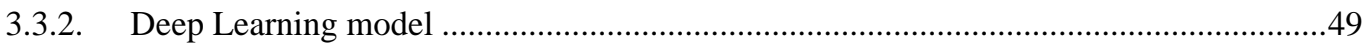

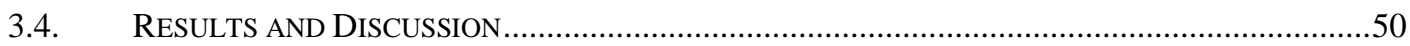

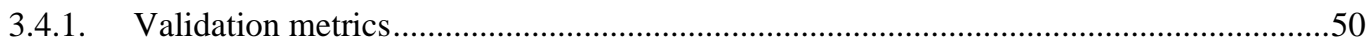

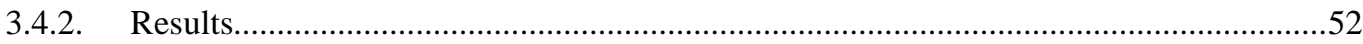

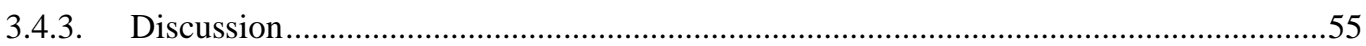

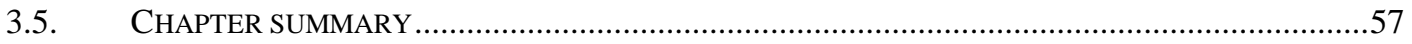

4. PRESSURE INJURY SEGMENTATION AND MEASUREMENT .....................................58

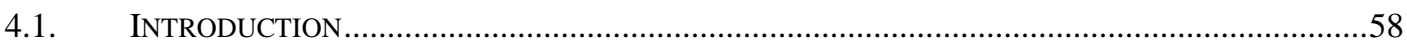

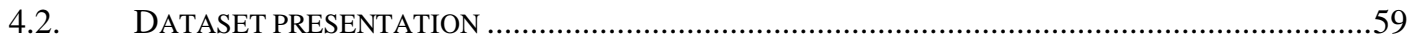

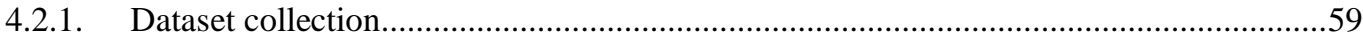

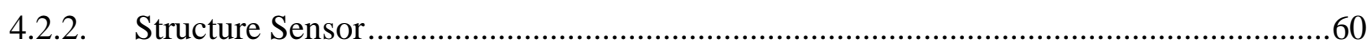

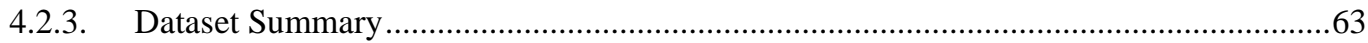

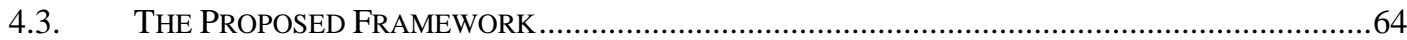




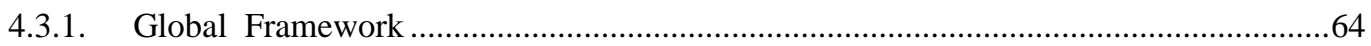

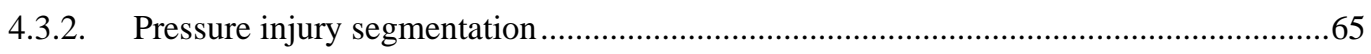

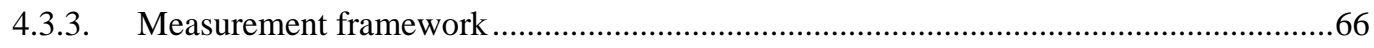

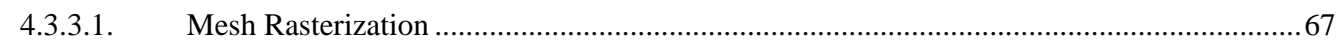

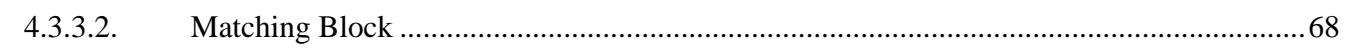

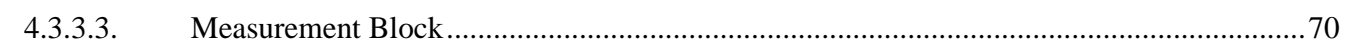

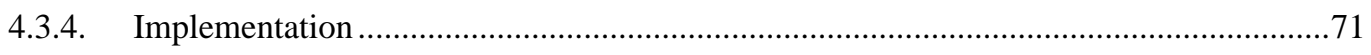

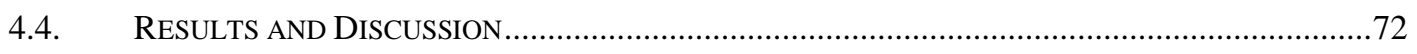

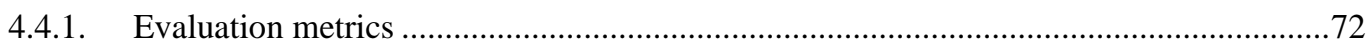

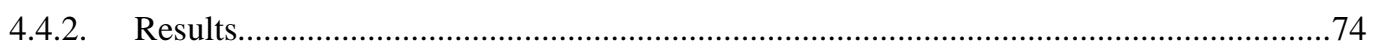

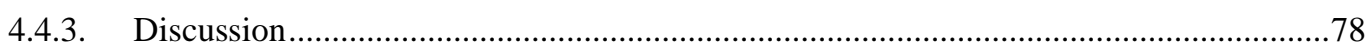

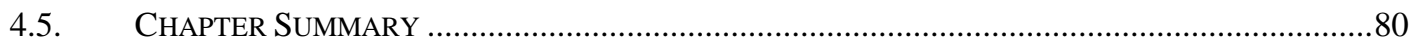

5. WEB-BASED APPLICATION ….........................................................................................................82

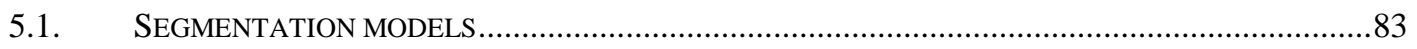

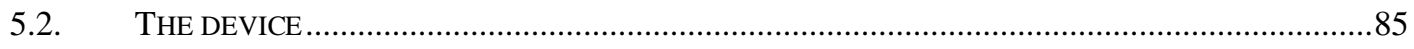

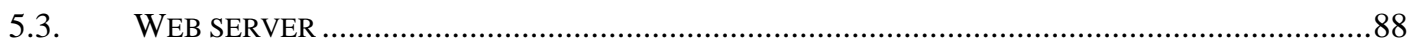

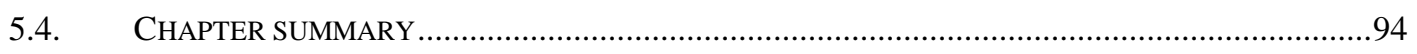

6. CONCLUSION AND FUTURE WORK ................................................................................................96

REFERENCES .........................................................................................................................101

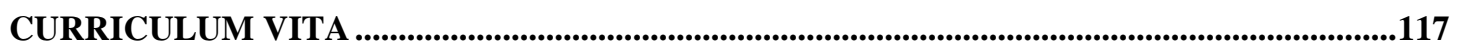




\section{LIST OF FIGURES}

Figure 1: The distribution of biomedical image analysis on different body parts using

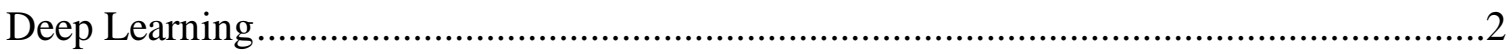

Figure 2: Global framework for pressure injury assessment ......................................

Figure 3: Research methodology followed in each one of the three study cases ...............5

Figure 4: Examples of Deep Learning application in medical imaging .........................12

Figure 5: Representation of a simple auto-encoder architecture (Adapted from Le, Q.V

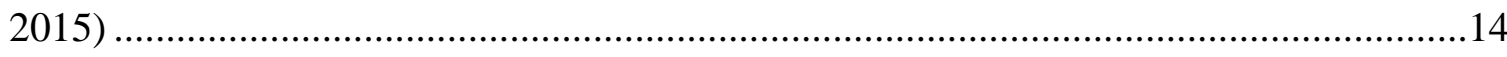

Figure 6: Illustration of the convolution layer with a filter size $3 \times 3$ and a zero padding 1

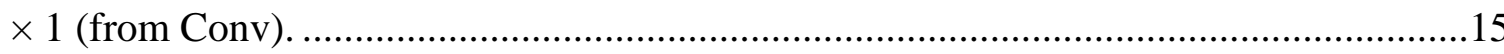

Figure 7:Representation of a convolutional neural network architecture containing 2

convolution layers, 2 pooling layers, a fully connected layer and an example of pressure

injury tissue type prediction (Adapted from $\mathrm{Lu} 2017$ )............................................16

Figure 8: Mask RCNN architecture overview ..................................................... 17

Figure 9: Illustration of 3D convolution applied on a $(9 \times 9 \times 9)$ input volume with a

$(3 \times 3 \times 3)$ filter sliding through the $3 \mathrm{D}$ space, and resulting in a $(7 \times 7 \times 7)$ output volume....18

Figure 10: Representation of a recurrent neural network architecture (Adapted from Le,

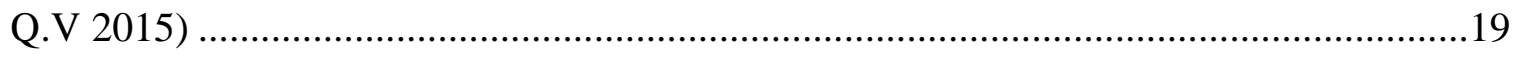

Figure 11: Risk factors of pressure injury occurrence ….......................................20

Figure 12: The main tissue types present in stage 3 and 4 pressure injuries. ..................21 
Figure 13: Evolution of DTPI to unstageable pressure injury.

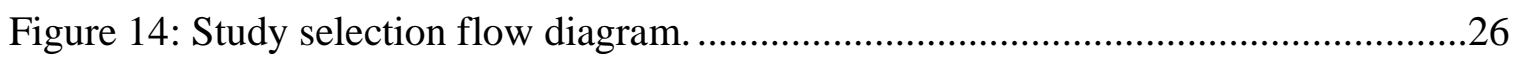

Figure 15: The analyzed articles in this review per country........................................27

Figure 16: The analyzed papers per wound/tissue classification image processing technique. (Please refer to (Zahia 2020b) for the corresponding referenced papers) ........29

Figure 17: The analyzed papers per wound segmentation image processing technique. (Please refer to (Zahia 2020b) for the corresponding referenced papers)

Figure 18: Collage of some pressure injuries segmentation results:

Figure 19: Collage of some pressure injuries Classification results. (a-c) wound tissue classification using SVM, proposed by Wannous et al. (Wannous 2011). (d-f) Wound tissue classification using SVM, proposed by Mukherjee et al. (Mukherjee 2014). (g,h) Pressure injury tissue classification using neural networks and Bayesian classifiers, proposed by Veredas et al. (Veredas 2010). (i) Lower extremity ulcer image classification using SVM and post-processing, proposed by Bochko et al. (Bochko 2010).

Figure 20: A set of pressure injury images and their corresponding ground-truth segmentation used to create the dataset and labels .45

Figure 21: Proposed architecture for image segmentation using Deep Learning technique

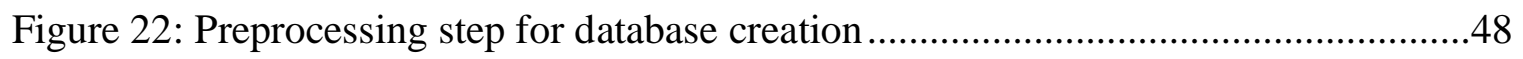

Figure 23: Dataset dictionary for the three different tissue types: Granulation, Necrotic .49 Figure 24: Proposed Convolutional Neural Network architecture for image segmentation. 
Figure 25: Illustration of different segmentation areas for performance metrics

measurement.

Figure 26: Examples of original images and their corresponding ground-truth and output segmentation using Deep Learning.......

Figure 27: Left:The Structure Sensor mounted on an iPad. Right: Scanning of a pressure injury using the Structure Sensor .60

Figure 28: Explanation of depth measurement using the Structure Sensor .61

Figure 29: An illustrative diagram of the composition of the database used to conduct this research. .63

Figure 30: Schematic view of our proposed framework.

Figure 31: Overview of Mask RCNN architecture chosen to segment the pressure injury: Proposals about the regions which possibly contain the wound based on the input image are detected. Then three branches predict the class of the object, the bounding box and the mask in pixels of the wound. Both stages are connected to the backbone structure. ..66 Figure 32:. Mesh rasterization process: the Obj file of the 3D mesh acquired with the Structure Sensor is rendered in order to retrieve the top view image and the matrix of face indices. .68

Figure 33: Matching illustration. (a) Point correspondence between 2D image and top view image, (b) 2D image, (c) 2D image after projective transformation to be aligned with the top view, (d) top view image.

Figure 34: Schematic view of the measurement block: after extracting the vertices belonging to the boundaries and the inside of the wound, the $3 \mathrm{D}$ mesh is rotated and the best fitting hyperplane is calculated to close the surface of the pressure injury. .70 
Figure 35: View of the mesh using BLENDER software

Figure 36: Examples of 3D mesh cropping of pressure injuries (Left: wound (a), Middle:

wound (b), Right: wound (c))

Figure 37: 3D mesh closing for volume measurement (Left: wound (a), Middle: wound

(b), Right: wound (c))

Figure 38: Results of the segmentation of the pressure injuries (Colors were generated randomly).

Figure 39:Measurement results of 15 different pressure injuries: depth in logarithmic scale $(\mathrm{cm})$.

Figure 40: Measurement results of 15 different pressure injuries: area in logarithmic scale $\left(\mathrm{cm}^{2}\right)$ .76

Figure 41: . Measurement results of 15 different pressure injuries: volume in logarithmic scale $\left(\mathrm{cm}^{3}\right)$ .76

Figure 42: Measurement results of 15 different pressure injuries: minor and major axes in logarithmic scale $(\mathrm{cm})$ .77

Figure 43: boxplot of the five components (expected vs. measured) .77

Figure 44:. Example of pressure injuries invalid for the measurement of the volume: Left and right columns present two pressure injuries located on the hip bone, whereas the middle column presents a pressure injury located on the heel and ankle

Figure 45: Image annotation illustration for necrotic tissue .83

Figure 46: Results of the segmentation of pressure injuries and rulers .84

Figure 47: The device for pressure injury data acquisition. .85

Figure 48: Authentification window .86 


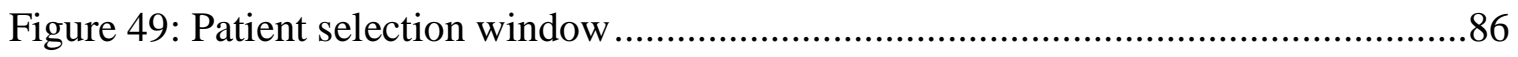

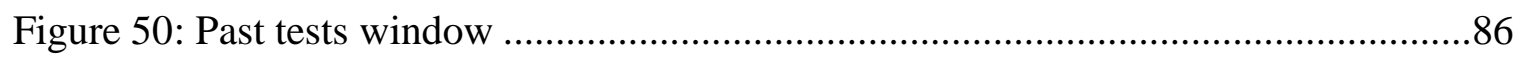

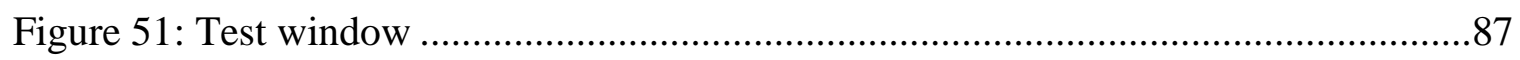

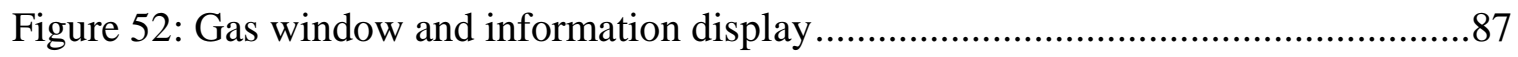

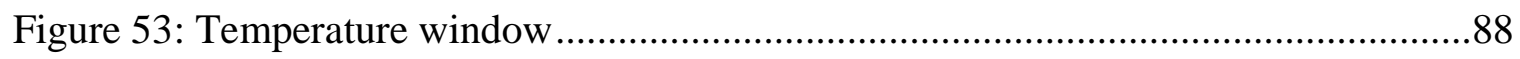

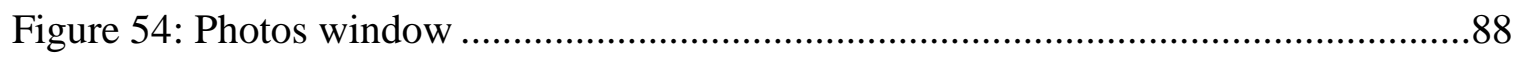

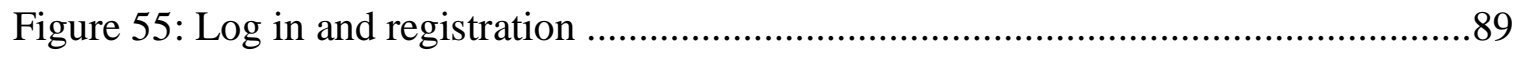

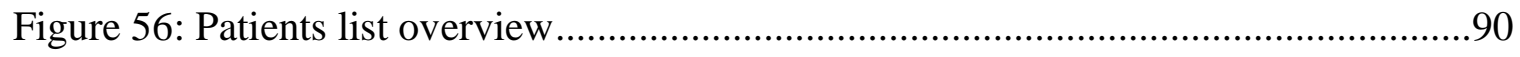

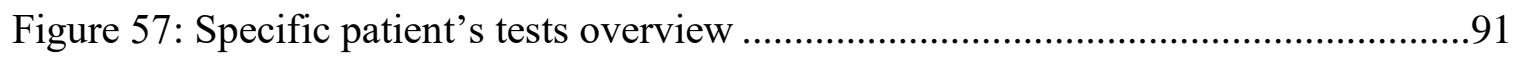

Figure 58: Illustration of image segmentation when "right image" is clicked on.............92

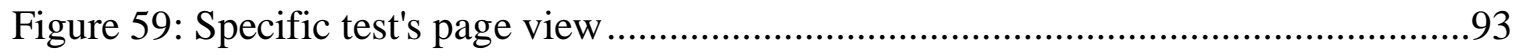

Figure 60: Three tissue types segmentation views ................................................93

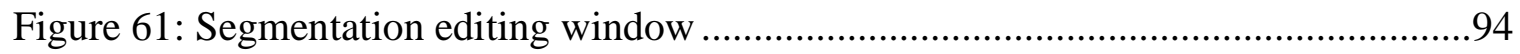




\section{LIST OF TABLES}

Table 1: Number of images for the training and testing of CNN architecture

Table 2: Results of the segmentation of image Fig. 25 (a) containing granulation, slough and necrosis 53

Table 3: Results of the segmentation of image Fig. 9 (b) containing only granulation.....53

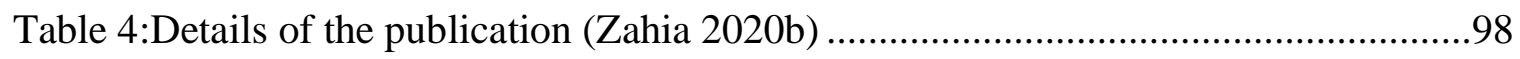

Table 5: Details of the publication (Zahia 2018) .....................................................99

Table 6: Details of the publication (Zahia 2020) .....................................................99 


\section{CHAPTER 1}

\section{INTRODUCTION}

In the last decade, Deep Learning (DL) techniques have gained popularity in many fields of applications such as: object detection and recognition, acoustic modeling, drug discovery and toxicology, speech recognition, language modeling, bioinformatics and many other research topics. In biomedical image analysis, DL approaches have been proven to be efficient in data analysis such as: Tissue/anatomy/lesion/tumor segmentation and classification, disease development prediction, images enhancement, etc. (Litjens 2017). Biomedical image analysis using Deep Learning was applied on several body parts including: Brain image analysis, Chest (x-ray and CT images), different pathologies, abdominal, cardiac, dermatological, Musculoskeletal, etc... as represented in Fig. 1 which includes the analyzed papers in (Litjens 2017) in addition to newer papers. However, in dermatology studies for instance, the usage of Deep Learning techniques remains limited for the assessment of skin wounds and more particularly for pressure injuries. The increasing prevalence and the associated economic cost of pressure injury 
care coupled with the negative impact of this type of wounds on the patient's life quality calls for the development of non-invasive tools and techniques that help in the design of more effective healing protocols. In this sense, image processing techniques have proven to provide suitable solutions towards this aim. As for other medical applications, Deep Learning still remains considered in its early stage, and researcher need to leverage their great effort and catalyze additional contributions in medical imaging in order to help the medical sector to serve the patients rapidly and efficiently.

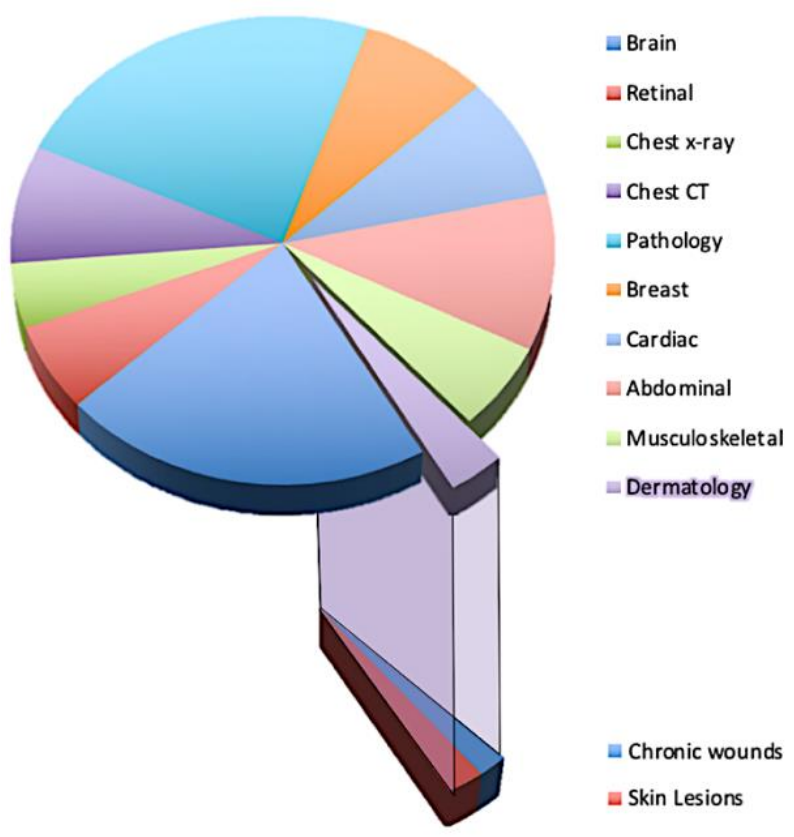

Figure 1: The distribution of biomedical image analysis on different body parts using Deep Learning

\subsection{Research hypotheses and objectives}

The analysis of the problem introduced throughout the previous section lead to the following hypotheses.

Combining Deep Learning and computer graphics techniques can create an efficient tool for an accurate assessment and analysis of pressure injuries. 
Based on the aforementioned hypothesis, this dissertation aims to profile the power of Deep Learning to tackle different medical imaging problems. In order to reach the main aim of this dissertation, the following specific objectives need to be fulfilled:

- SO1: To construct a full database containing 2D and 3D images of the pressure injuries, in order to ensure an optimal data quality for a high efficiency of the system, by making several study visits in collaborating hospitals and patients' homes.

- SO2: To define the current state of the art achievements in pressure injury imaging systems. This objective is fulfilled by the comprehensive review of the existing techniques for assessing pressure injuries using imaging technology.

- SO3: To design and implement algorithms for the segmentation and classification of pressure injuries, and to combine 3D representations with Deep Learning techniques for quantitative characteristics extraction (Area, surface, perimeter, depth, volume...). The following framework summarizes the idea behind this case study of pressure injury assessment, as shown in figure 2 .

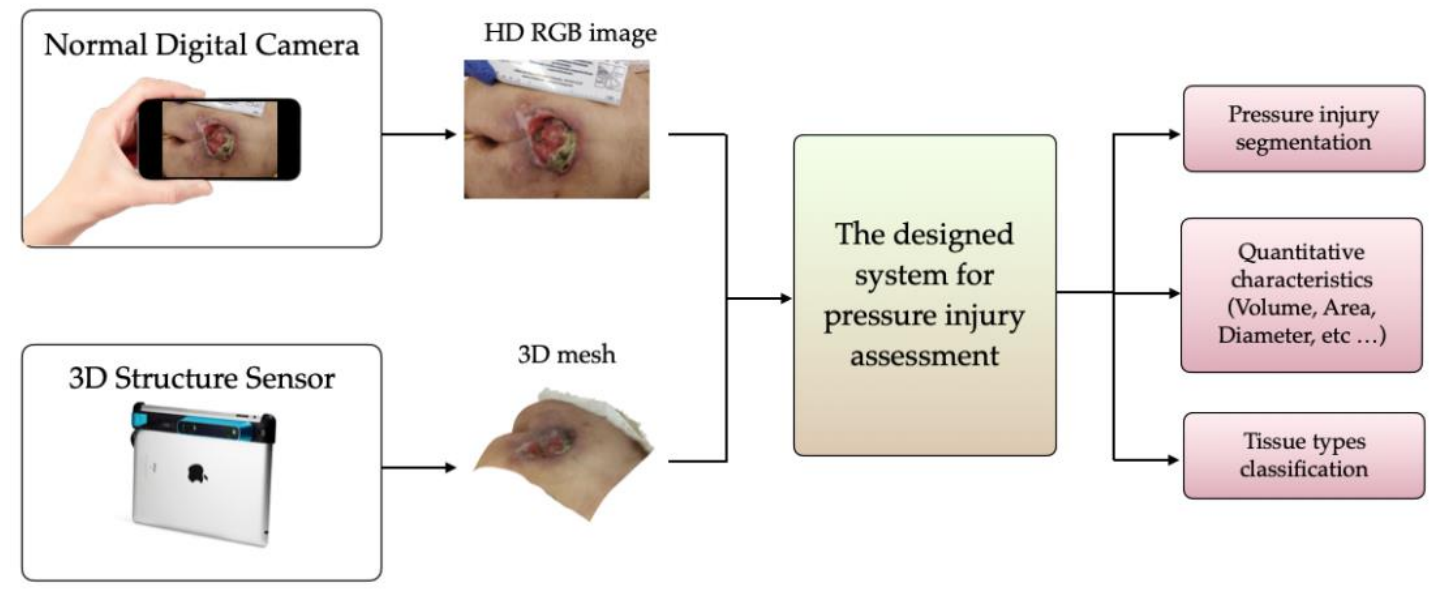

Figure 2: Global framework for pressure injury assessment 


\subsection{Scientific and social impact and contribution}

The use of Deep Learning techniques is believed to improve the diagnosis of several health issues, using all different type of data and modalities. The issue with traditional approaches is the necessity to choose the features which are important in the given images. Hence, the computer vision engineers need to judge with a trial and error process to decide the features which best describe the objects to detect. Deep Learning has introduced the concept of end-to-end learning. When the machine is given a set of images, which have been annotated by experts, Neural networks discover the underlying patterns during the training and automatically find out the most descriptive features with respect to the objects to be detected. In fact, in the last decades, Deep Learning has become the dominant method for many machine vision-based applications such as object detection, object localization and semantic segmentation.

Besides using Deep Learning to tackle the studies medical problems, the other main part of this thesis work is the combination of more than one input data to reach the aimed objectives. Not only 2D photographs were used to design the overall system, but also 3D meshes which contain essential geometric information were used to reach an accurate assessment and healing evaluation of pressure injuries. Most hospitals require documentation of the pressure injury using images, and additionally, expect the healthcare professionals to take more detailed information. Using such system, they will have the ability to use the time with the patient more effectively.

\subsection{Research methodology}

This section explains the methodology followed by the research work of this 
dissertation. This dissertation has been submitted on a PhD by publication basis, so the dissemination of scientific publications is presented throughout the whole research process, as it is displayed in Fig. 3.

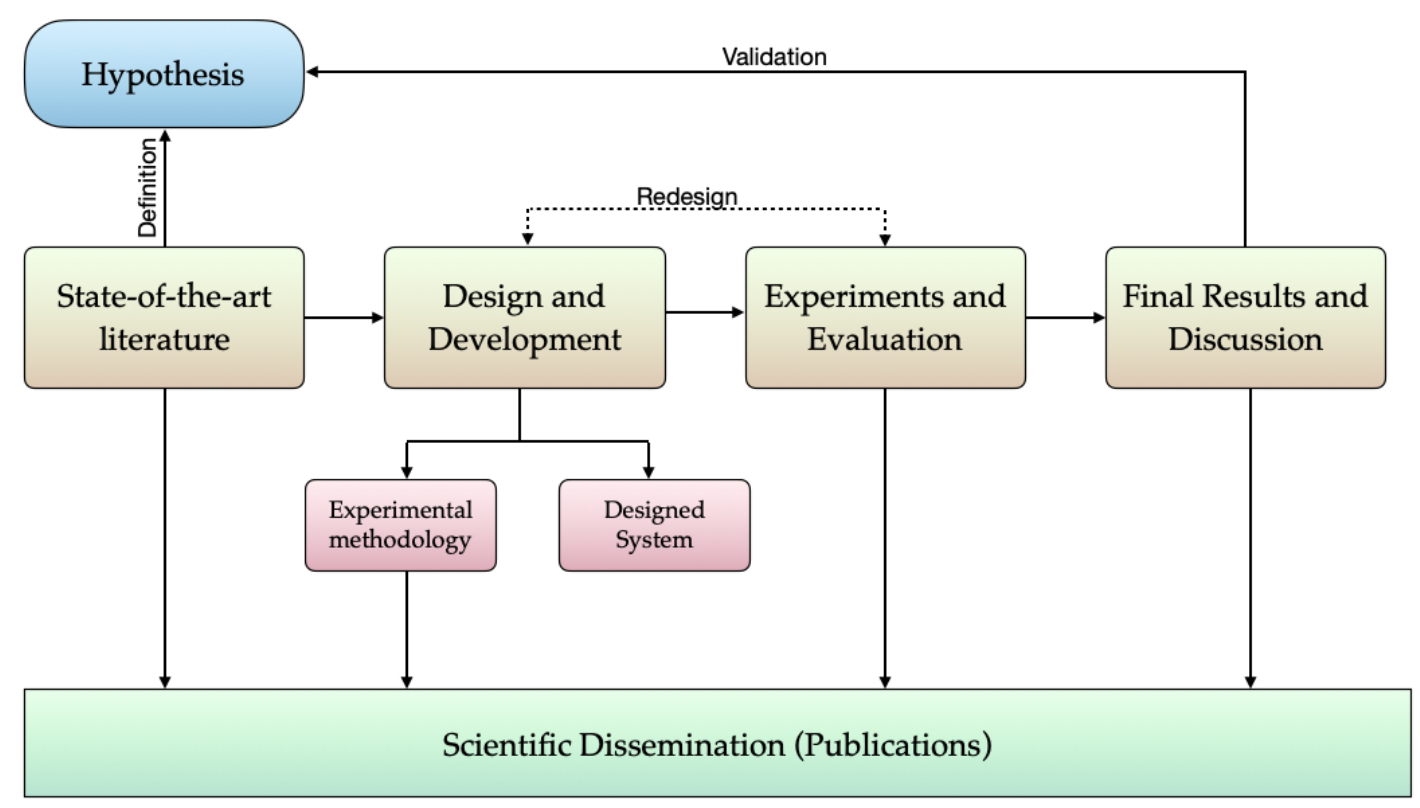

Figure 3: Research methodology followed in each one of the three study cases

- State-of-the-art literature: The main objective of this step is to analyze and understand the current state of the art of all the technologies and techniques involved in the problem in hand. The knowledge obtained during this stage will lead to the formulation of the hypothesis and later to the comparison with the obtained results.

- Design and Development: After the literature analysis and the processing of the knowledge acquired in the previous step, this step involves the design and development of the different parts of the system. 
- Experiments and Evaluation: At this stage, methods for the evaluation of the system are

defined. the designed system is redesigned depending on its performance on the given validation data.

- Final results and discussion: This step aims to compare the obtained results with the state-of-the-art results, which leads to the final assessment of the established hypothesis.

\subsection{Summary and organization of the study}

This section outlines the structure and content of the different chapters that are part of this dissertation. The chapters described below contain the major content of published articles in peer reviewed journals with impact factor.

- Chapter 1 - Introduction: This first chapter has introduced the overall concept of the outlined research study. Its main objective is to set the main objectives and the research scenario. It will leave the reader with an overall idea of the conducted research study and system development. On top of this, the hypothesis and specific objectives introduced in this section will be key factors in the overall development of the study.

- Chapter 2: Literature review: This chapter presents the state-of-the-art techniques for the assessment of pressure injuries and chronic wounds and the advances of Deep Learning in medical imaging applications.

- Chapter 3: Tissue types classification: The second part presents the segmentation and classification of pressure injury tissue types using convolutional neural networks. This chapter illustrates the proposed framework with the initial 
dataset (before starting hospitals study visits and database creation).

- Chapter 4: Pressure injury segmentation and 3D measurement: In this chapter, we present the acquired dataset of 2D photographs and 3D meshes from collaborating hospitals. Then we present the designed end-to-end system where the segmentation of pressure injuries is conducted using a pretrained Mask RCNN model. Then, the output of the segmentation is combined with 3D meshes in order to automatically extract all the measurements necessary for an efficient assessment of these wounds (area, depth, volume, perimeter, etc.).

- Chapter 5: Web-based application: The fifth chapter presents the integration of image processing techniques in a web-based application, which contains the data acquired from a sensor device designed for a non-intrusive assessment of pressure injuries and infection detection. By the means of this application, the doctors can easily track the evolution and healing of the wound through the time using different visualization graphs.

- Chapter 6: Conclusion: The sixth and final chapter of the dissertation introduces the different thoughts and conclusions extracted from the final evaluation of the research work presented. and whether the objectives set in the beginning were successfully met during the process. Future lines will be discussed in this section. 


\section{CHAPTER 2}

\section{LITERATURE REVIEW}

Owing to advances in high-throughput technologies, important amounts of medical data has been collected in recent decades, including data related to medical images (Amoon 2020). Traditionally, discriminative features were manually designed and extracted for classification and detection of abnormalities, and segmentation of regions of interest in different medical applications (Xu 2020). This step requires the expertise of physicians and experts. However, because of data complexity and the limited knowledge in data interpretation, Deep Learning has soon caught the attention of researchers as one of its powerful advantages is the unnecessity of feature selection to reach the final goal. Deep Learning models are composed of multiple processing layers to learn representations of the data with multiple levels of abstraction. These methods discover complicated structures in large data sets. Thus, they have dramatically improved the state-of-the-art in many fields of machine learning (Zahia 2020b). The most successful type of models for image analysis to date are convolutional neural networks (CNNs). CNNs contain many layers that 
transform their input with convolution filters of a small extent. Other architectures are also widely used for data analysis such as Recurrent Neural Networks (RNNs) which were developed for discrete sequence analysis and their variations such as Long Short Term Memory (LSTM) and Gated Recurrent Unit (GRU).

In the following sections we will present Deep Learning for medical imaging application along with the corresponding contributions with great impact in the field. Then we will present the widely used Deep Learning architectures used in the literature to tackle the aforementioned applications. Then, we will present the state of the art advances in the special study case of pressure injuries.

\subsection{Biomedical imaging modalities}

There are several types of biomedical images which depend on the acquisition technique and the clinical analysis desired from the specific part of the human body. The list below shows the types of medical images most widely used in the research field.

\subsubsection{Clinical images}

These images are digital images of specific parts of the patient's body, which are usually acquired to track the efficiency of the treatment in case of a skin lesion or a wound over time. The widespread use of digital devices has made the clinical images more available and easily shared in order to improve the diagnosis, the treatment, the management, the clinical practice and inter-clinician communication.

\subsubsection{X-ray imaging}

$\mathrm{X}$-Ray imaging is one of the widely used biomedical imaging modality to

capture the inside of the body. The images are composed of different shades of black and 
white, as each body tissue absorbs the radiation differently. Bones for instance appear white as they absorb most of the rays because of the calcium present in them. The most familiar usage of such image is to check bone fractures or to detect common diseases in chest such as pneumonia.

\subsubsection{Magnetic Resonance Imaging}

The MRI modality is a powerful technique for the diagnosis of abnormalities in soft tissues. Using strong magnetic field together with radiofrequency waves, it is able to capture physiological processes in the body. For instance, MRI is used to detect some brain lesions and tumors, and can construct the images in all the three axes. There are different types of MRI: Diffusion MRI used the diffusion of eater molecules to generate contrast in the images. This type mostly used to detect multiple sclerosis, epilepsy and Alzheimer's diseases (Bodini 2009). The changes in neural activity can be diagnosed using Functional MRI (fMRI),

\subsubsection{Computed Tomography (CT)}

Tomography aims at capturing sectional images of the internal organs, bones, soft tissues and vessels which are then superimposed in order to form three-dimensional images. This type of imaging is commonly used to detect abnormal tumor growth, artery diseases and blood clots.

\subsubsection{Ultrasound Imaging}

Ultrasonography is an imaging technique which uses high-frequency sound waves to visualize real-time internal body structures. It is mostly used to monitor the developing fetus during pregnancy. The constraint with this imaging technique is the inability to capture organs blocked with air or air filled such as lungs. 


\subsubsection{Microscopic Imaging}

Microscopy is an imaging technique used to analyze tissues in the microscopic level. Thanks to the acquisition of microscopic and pathologic images, practitioners and medical researchers are able to improve cell pathology diagnosis such as cancer detection. The analyzed tissues are usually coming from a biopsy and are then dyed with staining elements for a better visualization at a cellular level.

\subsection{Deep Learning for medical imaging}

Medical imaging literature has witnessed great progress in the designs and performance of deep convolutional models for medical image segmentation (Tajbakhsh 2020). Also, image classification was one of the first areas in which Deep Learning made a major contribution to medical image analysis. Litjens et al. (Litjens 2017) surveyed the early Deep Learning solutions for various medical imaging applications including image classification, object detection, and object segmentation. Biomedical image analysis using Deep Learning was applied on several body parts including: Brain image analysis (Dou 2015)(Ghafoorian 2017)(Ismael 2020)(Akil 2020)(Shi 2017), Chest (x-ray and CT images) (Wang 2016a)(Dou 2016b), different pathologies (Chang 2017), abdominal (Lu 2017), cardiac (Gulsun 2016), dermatological (Zahia 2018)(Zahia 2020), etc. The huge amount of contributions in the field of biomedical data analysis with artificial intelligence only confirms that using AI to examine the healthcare sectors will improve patient assistance and patient care in the future direction (Xu 2020). The figure below (Fig. 4) illustrates the applications of Deep Learning in biomedical image analysis. 

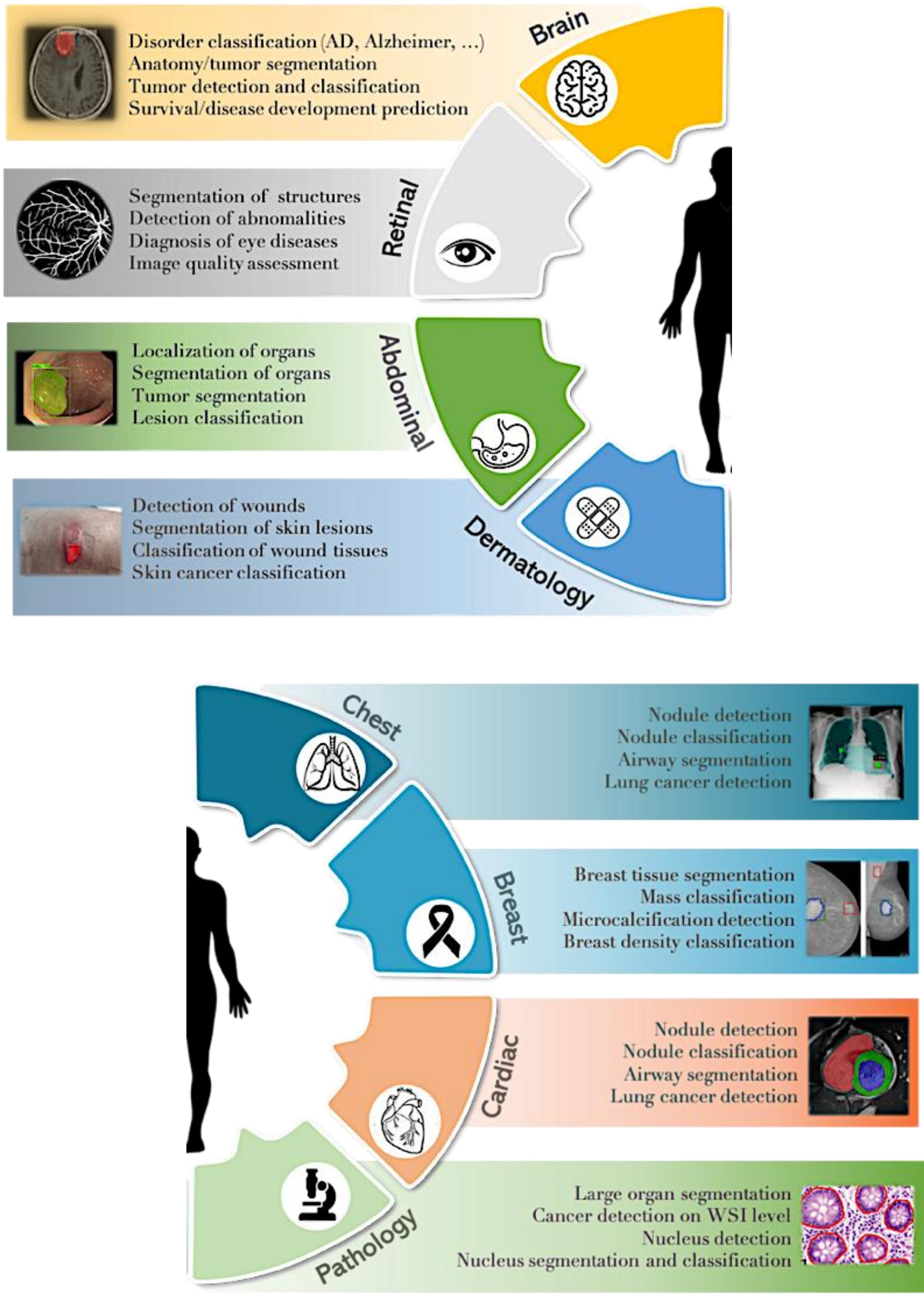

Figure 4: Examples of Deep Learning application in medical imaging 


\subsubsection{Deep Learning architectures}

Deep learning architectures have revolutionized the analytical aspect for big data and have omitted one of the major steps in classical machine learning methods which was features extraction. These architectures consist of deep networks of varying topologies, made of several layers that process the data: input layers which are given the input the data, the hidden layers where data is processed and combined, then an output layer which produces the desired output (prediction). The number of hidden layers define the depth of the architecture. Activation functions such as Sigmoid, Rectified Linear Unit, Hyperbolic tangent and others map the non-linearity relationship between the input and output. This can help the network learn complex data and provide accurate predictions. There are several architectures which have been used in biomedical image analysis:

\subsubsection{Auto-encoders (AES)}

An auto-encoder is an unsupervised learning algorithm that learns to produce the same output as the one given in the input, while using fewer neurons in the hidden layer, as shown in Fig. 5. It is composed of two stages: Encoder and decoder. The encoder part learns the features of the input with fewer parameters, and therefore, it reduces its dimensionality. On the contrary, the decoder part, generates the output vector as a representation of the compressed vector in the hidden layer (Le, Q.V 2015). When the auto-

encoder has multiple hidden units, it is named stacked auto-encoder (SAE). This type of architecture has also been used in biomedical image analysis and has been proven to be efficient in several image processing tasks such as the classification of Alzheimer's and Mild Cognitive Impairment diseases (Suk 2013)(Suk 2015), features extraction of rs-fMRI 
for Mild Cognitive Impairment diagnosis (Suk 2016), image similarity metric to learn the correspondence of two images (Cheng 2018).

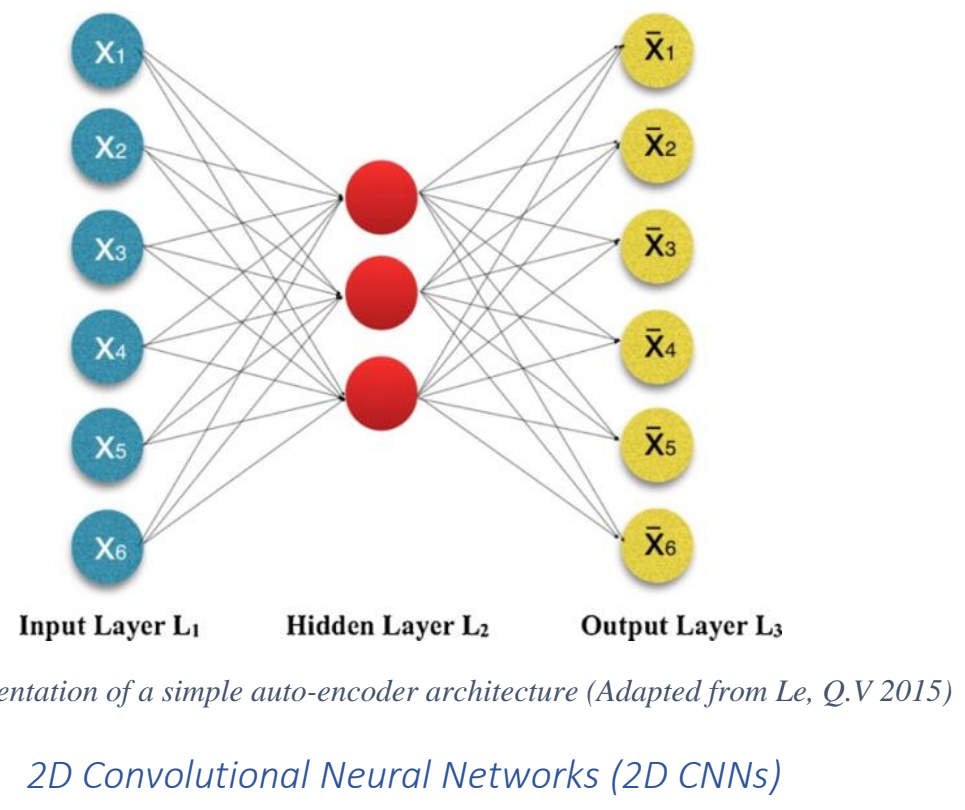

Convolutional neural networks (CNNs) represent the most common Deep Learning architecture used for biomedical image analysis, and have been proven to be very efficient in patterns recognition and image segmentation (Zahia 2018)(Ronneberger 2015). Also known as ConvNets, they are feed-forward neural networks composed of cascaded convolution and pooling layers, optionally followed by fully connected layers (Lu 2017).

- Convolution layer: This layer represents the main concept behind convolutional neural networks. It is based on convolving an input image with kernels to obtain feature maps. In fact, as the filter moves along the input image, it uses the same parameters for the convolution (Fig. 6). Hence, the formed feature map characterizes a specific pattern in the image. In this sense, $\mathrm{CNN}$ is able to recognize specific patterns and is robust to distortions and geometric transformations. The convolution layer has many features. The filter size defines the size of the subregions being convolved. The number of filters 
represents the number of channels in the convolution layer. The stride defines the step size with which the filter moves along the image.

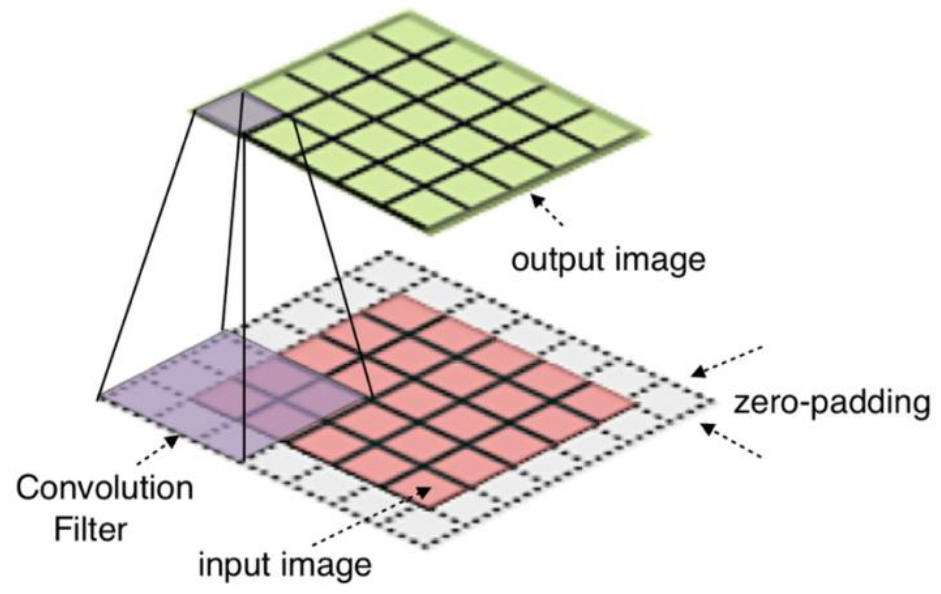

Figure 6: Illustration of the convolution layer with a filter size $3 \times 3$ and a zero padding $1 \times 1$ (from Conv).

- Pooling layer: This layer minimizes the number of parameters used in the network by resizing the previous layer. It returns the maximum or mean value of a subregion of the previous layer.

- Fully Connected Layer: This layer connects all its neurons with all the previous neurons. For classification purposes, this layer generates the final output which is equal to the number of the type studied.

- Softmax layer: known as normalized exponential, this is an activation function which follows the fully connected layer for multi-class classification purposes.

- Classification layer: this assigns the output result of the Softmax layer to one of the studied classes by using the cross entropy function.

Since convolutional neural networks are generally used for image processing, the illustration of this architecture will be presented using an image as 
the input. Fig. 7 depicts an example of a CNN architecture which is composed of 2 convolution layers, 2 pooling layers, a fully connected layer and output predictions of 5 studied classes.

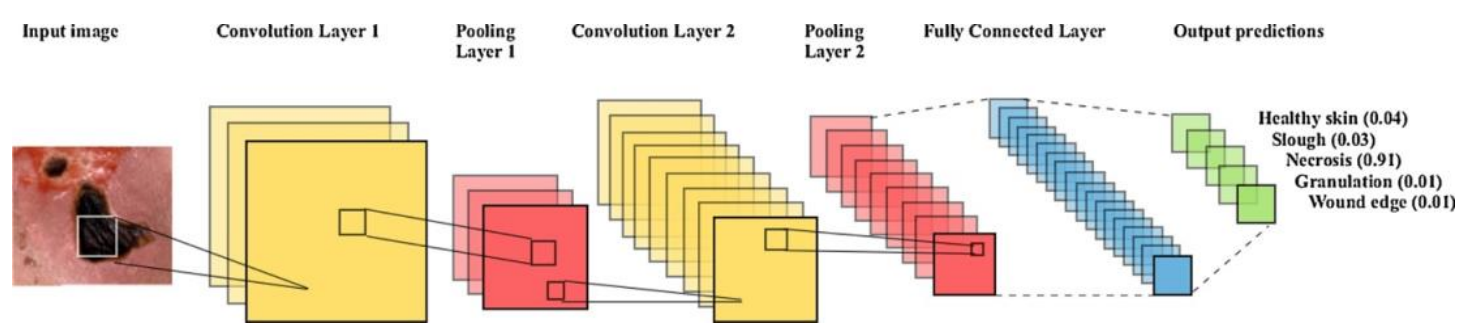

Figure 7:Representation of a convolutional neural network architecture containing 2 convolution layers, 2 pooling layers, a fully connected layer and an example of pressure injury tissue type prediction (Adapted from Lu 2017).

\subsubsection{Region Based CNNs:}

(R-CNN, Fast R-CNN, Faster R-CNN and Mask R-CNN): For object detection task, these architectures have had a tremendous impact in research. R-CNN as well as Fast RCNN enable the detection of the object using a bounding box. Since R-CNN (Girshick 2014) was computationally expensive because it was using selective search to look for the highest probability of containing the object, an improvement was made in the next architecture Fast R-RCNN (Girshick 2015) where the image was fed to a ConvNet to extract features of the region proposals and then fully connected layers before the classification output. Lastly, Faster R-CNN (Ren 2015) outperformed the aforementioned architectures by reducing the complex pipeline that they exhibited. In fact, a region proposal network was inserted after the last convolutional layer. Faster R-CNN has become the most used architecture for object detection problems. Later, Mask R-CNN (He 2017) was released, enabling the performance of semantic segmentation. This architecture 
extends Faster R-CNN by adding a branch for predicting segmentation masks on each Region of Interest (RoI), in parallel with the existing branch for classification and bounding box regression, as shown in Fig. 8 .

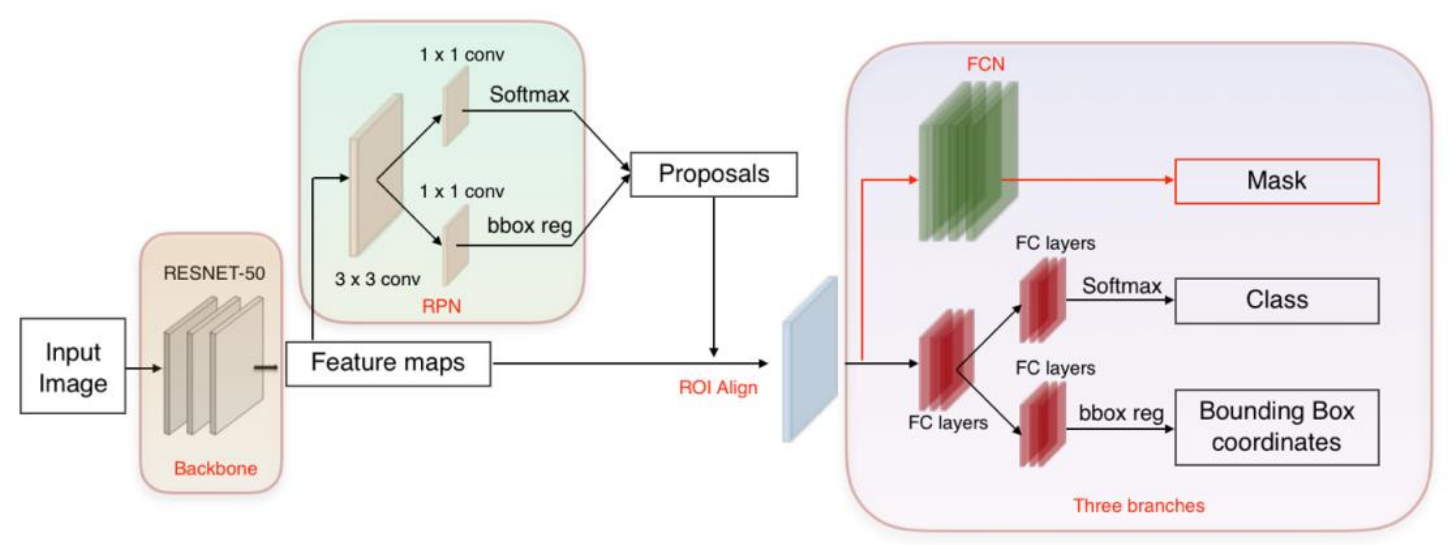

Figure 8: Mask RCNN architecture overview

\subsubsection{3D Convolutional Neural Networks (3D CNNs)}

The 3D CNNs function in the same way as 2D CNNs, with the exception of convolution kernels that are expanded to three dimensions. The architecture of 3D CNN is composed of stacked layers of 3D convolutions and 3D maxpooling layers, followed by flatten layer, dense layers and output prediction layer.

- 3D convolution layer: It represents the core layer of a CNN architecture.

A filter with learnable weights slides over the input while moving in 3-direction (height, width, depth of the image) to calculate the feature representations, and produces a weighted sum as the output. The weighted sum is the feature space that represents the input for the next layers. Figure 9 shows the concept of the sliding $3 \mathrm{D}$ window of size $(3 \times 3 \times 3)$ over a $(9 \times 9 \times 9)$ matrix with stride $=(1,1,1)$ and no zero padding. The output size is $(7 \times 7 \times 7)$. 


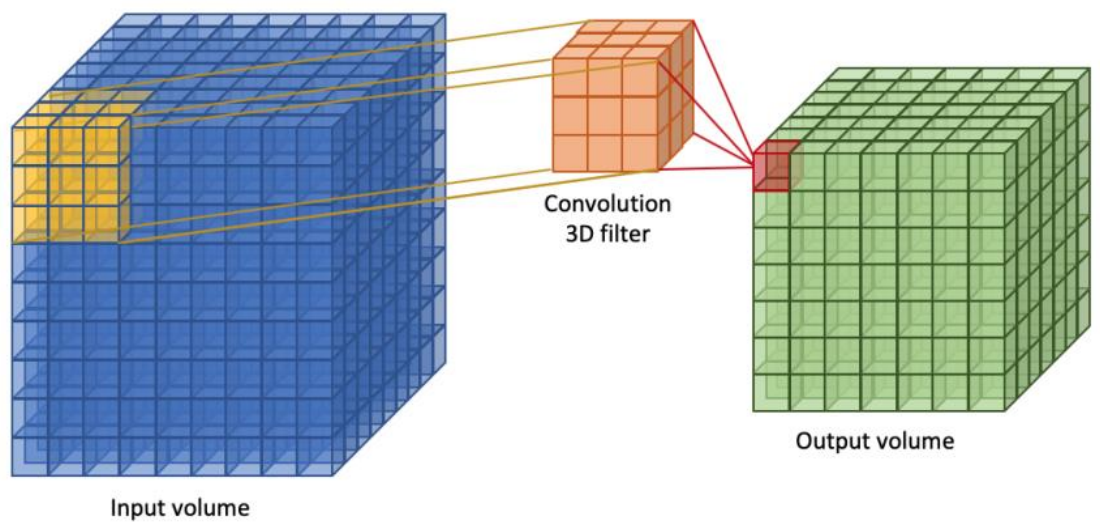

Figure 9: Illustration of $3 D$ convolution applied on a $(9 \times 9 \times 9)$ input volume with a (3x3x3) filter sliding through the $3 D$ space, and resulting in a ( $7 \times 7 \times 7)$ output volume

- 3D maxpooling layer: This layer extends the functionality of a max pooling layer to a third dimension. It down-samples the input data by dividing it into cuboidal regions and computing the maximum of each region. The purpose of its usage is to gradually decrease the spatial size of the representation and the amount of parameters learned in the network, and hence controlling overfitting.

\subsubsection{Recurrent Neural Networks (RNNs)}

The important contributions of RNNs are in the areas of language modeling, image labeling and speech processing and prediction. In such fields, the output is highly correlated with previous data. Hence, the systems should not process the data independently but as a whole sequence. For this very reason, Recurrent Neural Networks are the most suitable to handle problems involving sequential data, such as time series data or sequences of characters and words. In fact, recurrent neural networks introduce loop or cycles, where the output of one layer is the input of the same layer or a previous one, as presented in Fig. 10. However, simple RNN architectures fall short of some processing needs. Actually, input data size is 
often large, therefore the range of context learned is quite limited, and so the gradients become very small to the point that they vanish to almost zero. This problem is referred as vanishing gradient problem (Hochreiter 2001). Long short-term memory (LSTM) networks overcome this shortcoming by adding cells, also called gates, to access past information. It enables efficient learning of long sequences by selecting the information to learn and the one to erase.

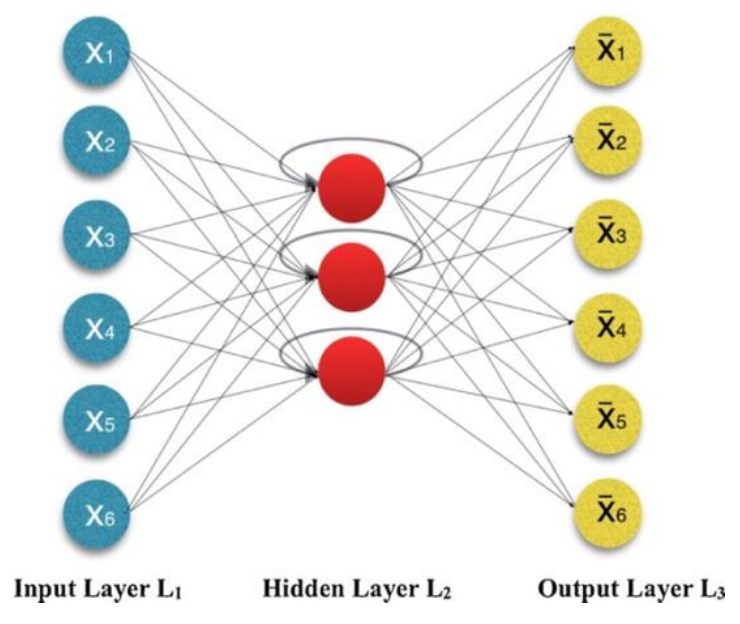

Figure 10: Representation of a recurrent neural network architecture (Adapted from Le, Q.V 2015)

\subsection{Pressure injury assessment}

\subsubsection{What is a pressure injury?}

The average life expectancy of world population has steadily increased during the last decades, and it is expected to continue growing during the next century. At the same time, medical professionals have witnessed an increasing incidence rate of diseases related to sedentary lifestyles and unhealthy eating habits, such as diabetes, especially in the Western diet (Klonoff 2009). The convergence of these two trends has increased the number of long time care patients subject to lengthy periods of immobilization who suffer pressure injuries: a type of chronic wounds resulting from damage caused by pressure over time causing an ischemia of underlying skin structures. These injuries appear most 
commonly at bony prominences like the sacral area and heel (Horn 2004). Some factors which contribute to pressure injury formation are: skin contact with a bed or a chair without frequent position changes, contact with urine or stool, diseases like diabetes that affect blood flow, injuries which restrict your body positioning and your nutritional status or medications. There are many other factors than may put the skin at risk for pressure injuries, as presented in Fig. 11.

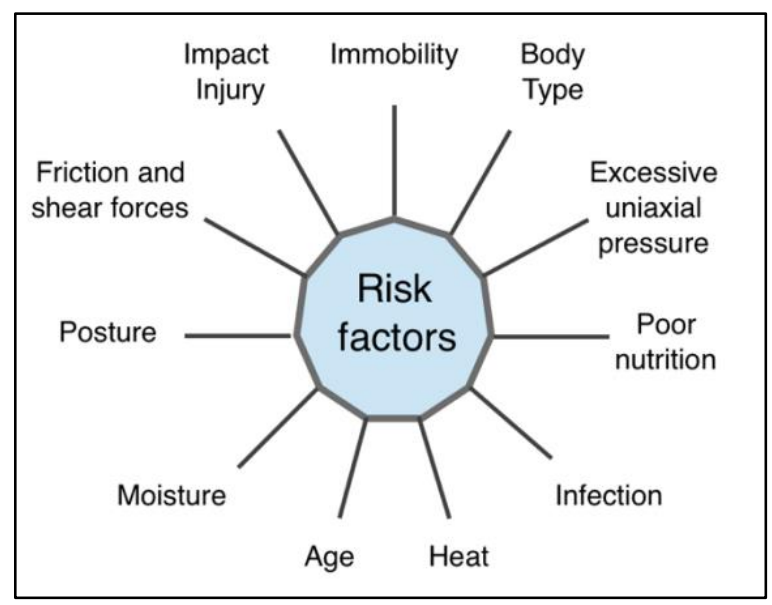

Figure 11: Risk factors of pressure injury occurrence

Despite all the prevention measures that are put in place, pressure injuries may still develop. More than 5.7 million people in the United States ( $2 \%$ of the population) develop chronic wounds, at an annual cost of US\$20 billion (Sen, C.K. 2019). And the risk of developing them during hospital stays is 3 times greater than the risk of being involved in a car accident. Healthy skin, muscle or fat tissue starts to die when blood flow is slowed or interrupted by pressure. This pressure can be caused by the bones pushing down on a surface like a chair or a bed.

Pressure injury stages are defined based on the deepest parts of the ulcer and the type of tissue affected. Higher stage ulcer represents deeper tissue damage and more serious injury. There are four main stages of pressure injuries (stages 2018): 
- Stage 1: Pressure injury often presents as intact skin with redness, and is usually over a bony area. The skin would stay red after the pressure is removed. This is because the first area affected by pressure is muscle tissue. In fact, due to its aerobic metabolism, muscle tissue requires more oxygen. Hence, when blood circulation is interrupted, lesions begin appearing in deep muscle levels before reaching the skin. The surface area may be painful, warm or softer compared to surrounding tissues.

- Stage 2: Pressure injury presents as an area where the top layer of the skin is missing with the open area being pink or red.

- Stage 3: Pressure injury is deeper and more severe than a stage 1 or 2 pressure injury. It goes into fat tissue, and dead tissue may also be present.

- Stage 4: Pressure injury is the most severe form of this wound. It is the deepest ulcer possible and would reach muscle tissue and possibly bones. Dead tissue may also be present with stage 4 pressure injuries. Fig. 12 depicts the main tissue types that can be present in stage 3 and 4 pressure injuries.

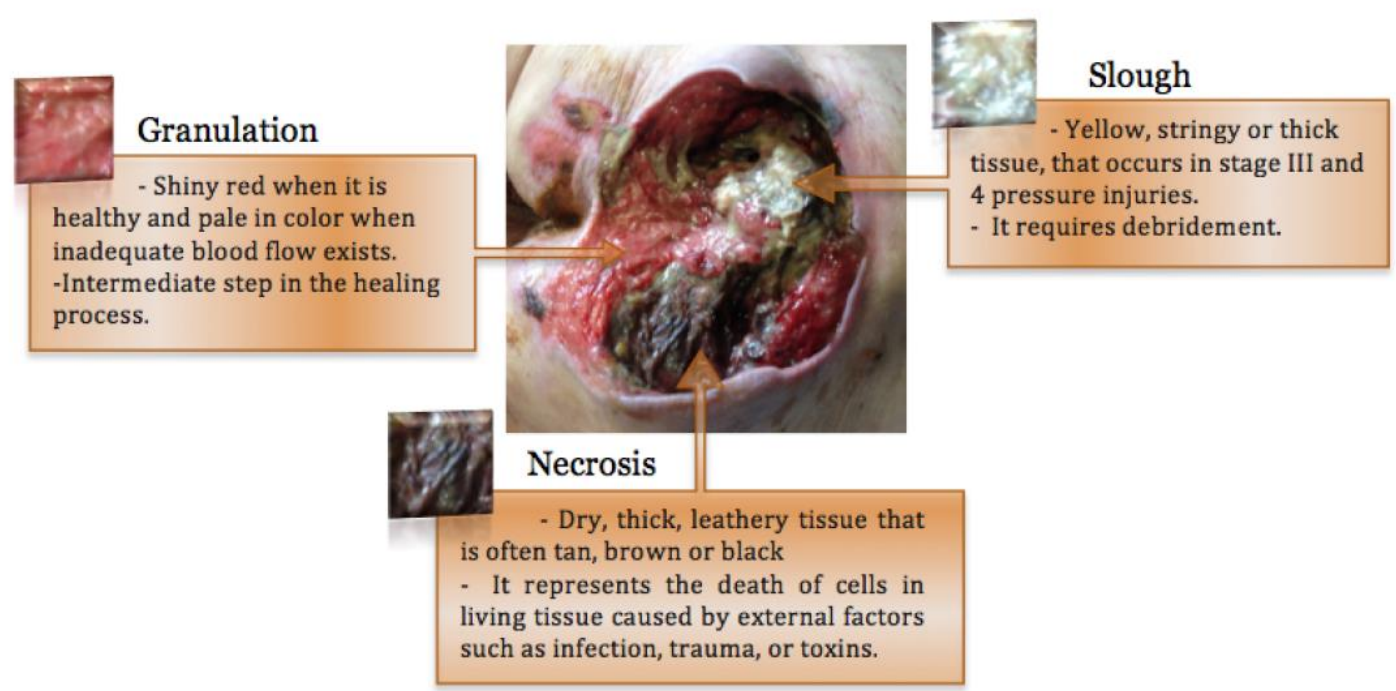

Figure 12: The main tissue types present in stage 3 and 4 pressure injuries. 
There are also two additional stages of pressure injuries. One is called unstageable because there are such exceedingly dead tissues in the wound that physicians cannot examine its depth. The other type of pressure injuries is called deep tissue pressure injury (DTPI). It usually begins with skin that is not broken, and it is usually purple or dark purple in color. This type of ulcer can open quickly and become a deep pressure injury (Cuddigan 2016), as it appears in Fig. 13.

Pressure injuries can occur in different areas, including: back or sides of the head, rims of the ears, shoulders, hipbones lower back, backs or sides of the knees, heels, ankles and toes. The age of the patient, coupled with certain medical conditions such as diabetes, slows down the healing of pressure injuries, which often are not healed before the death of the patient (Brown 2003), and represent a significant economic cost for medical care services (Soldevilla 2016). Pressure injuries are both painful and prone to infection, which calls for a continuous monitoring of their evolution by medical staff.

Day 1

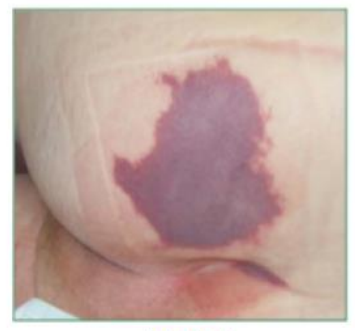

DTPI
Day 3

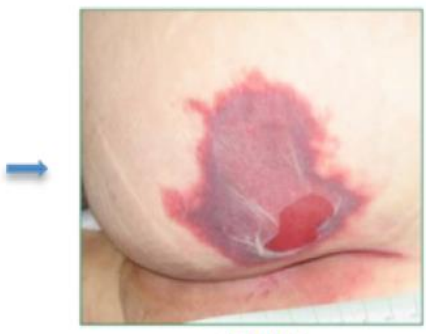

DTPI
Day 10

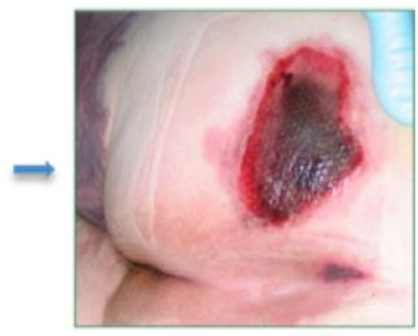

Unstageable

Figure 13: Evolution of DTPI to unstageable pressure injury.

Documenting the pressure injury once the patient is admitted to the hospital is the first essential step in the process of prevention and assessment. Firstly, caregivers need to check if the patient has already an existing pressure injury. In such case, documenting the 
stage, the size, the color, the drainage and other characteristics is the first step towards a properly done management of the pressure injury. In case the state of the wound is severe, wound care team is to be contacted to prescribe a personalized treatment. This includes reducing pressure and friction by repositioning every one to two hours and using support surfaces to protect sensitive skin and minimize shear, cleaning and dressing the wound depending on its stage, and removing damaged tissue. The latter intervention is crucial to keep the wound free of infected or dead tissue. Depending on the state and stage of the pressure injury, the treatment may differ. When the wound is in its first stages, a gentle cleaning and proper dressing is required to prevent it from getting infected. When the pressure injury is in stage 3 or 4 , it may take several months to heal, or may never do, especially if other risk factors are permanently present, such as: poor blood circulation and vascular diseases, diabetes, poor nutrition, immobility. Hence, a user-friendly system for a non-intrusive assessment of pressure injuries would represent a valuable tool for frequent assessment and monitoring of these wounds.

To avoid the discomfort of patients in the process, non-invasive wound monitoring techniques are preferred. In this sense, imaging techniques are called to play a key role, as they allow an accurate analysis of its features with no need of contact with the wound itself.

\subsubsection{Wound imaging with machine learning}

The studies of skin wounds using image processing have skyrocketed during the beginning of the 21 st century. Hence, the majority of the presented contributions were published during the last decade. 


\subsubsection{1. $\quad$ Searching methodology}

The literature search was performed using several databases, namely: Web of Science, SpringerLink, IEEE Xplore, PubMed, Science Direct, the Scientific Electronic Library Online (SciELO), SPIE Digital Library and Wiley Online Library.

\section{Searched Terms}

In order to look for papers regarding wound analysis using image processing techniques, different search terms were used, and some of them were combined in the same search. Furthermore, the terminology of pressure ulcer was changed to pressure injury in April 2016 by the National Pressure Ulcer Advisory Panel (NPUAP) (NPUAP 2016). Therefore, both terminologies were included in the search terms. The authors used the following terms: "image processing", "image analysis", "medical diagnostic imaging", "pressure ulcer", "pressure injury", "skin lesion", "skin wound", "wound measurement", "tissue classification", "wound seg- mentation", "wound healing", "wound area measurement", "wound boundary delineation", "monitoring systems", "3D modeling", "volume measurement", "teleMedicine", "surface reconstruction", "machine learning", "Deep Learning”, “wound evaluation", "diabetic foot”, “wound assessment”, “neural networks", "feature extraction", "pattern recognition", among others.

\section{Inclusion Criteria}

Titles and abstracts represented the first selection step to find the papers meeting the desired criteria. Then duplicates were removed. Since wound assessment involves several applications such as wound segmentation, tissue classification, wound measurement and healing prediction, the corresponding papers which were judged to be 
suitable were retrieved and classified according to the assessment application, using the Zotero application.

\section{Exclusion Criteria}

Papers not specifically dealing with wound imaging techniques, or lacking precision were excluded from the study.

Fig. 14 depicts the study selection flow diagram including the number of papers addressing each one of the wound imaging problems:

- Wound segmentation: The pressure injury segmentation problem consists in automatically finding the boundaries limiting the wound, so that it can be separated from the rest of the image for further analysis [17].

- Wound measurement: Once the wound is segmented, the following analysis often consists in conducting measurements of physical characteristics of the ulcer, such as its area, depth and volume, sometimes involving the creation of volumetric models of the wound.

- Tissue classification: In advanced stages of pressure injury evolution, subcutaneous tissues become visible (e.g. tendons, muscles, bone). In this situation, a typical analysis consists in classifying the different types of tissues visible.

- Healing evaluation: Finally, coupling the results of pressure injury image analysis with medical domain knowledge allows performing pressure injury healing evaluation. 
Databases Searched: Web of Science, SpringerLink, IEEE Xplore, PubMed, Science Direct, the Scientific Electronic Library Online (SciELO), SPIE Digital Library and Wiley Online Library

Search terms: pressure injury, wound segmentation, tissue classification, monitoring systems, Machine learning, Deep Learning, pattern recognition,...

Total papers retrieved: 199

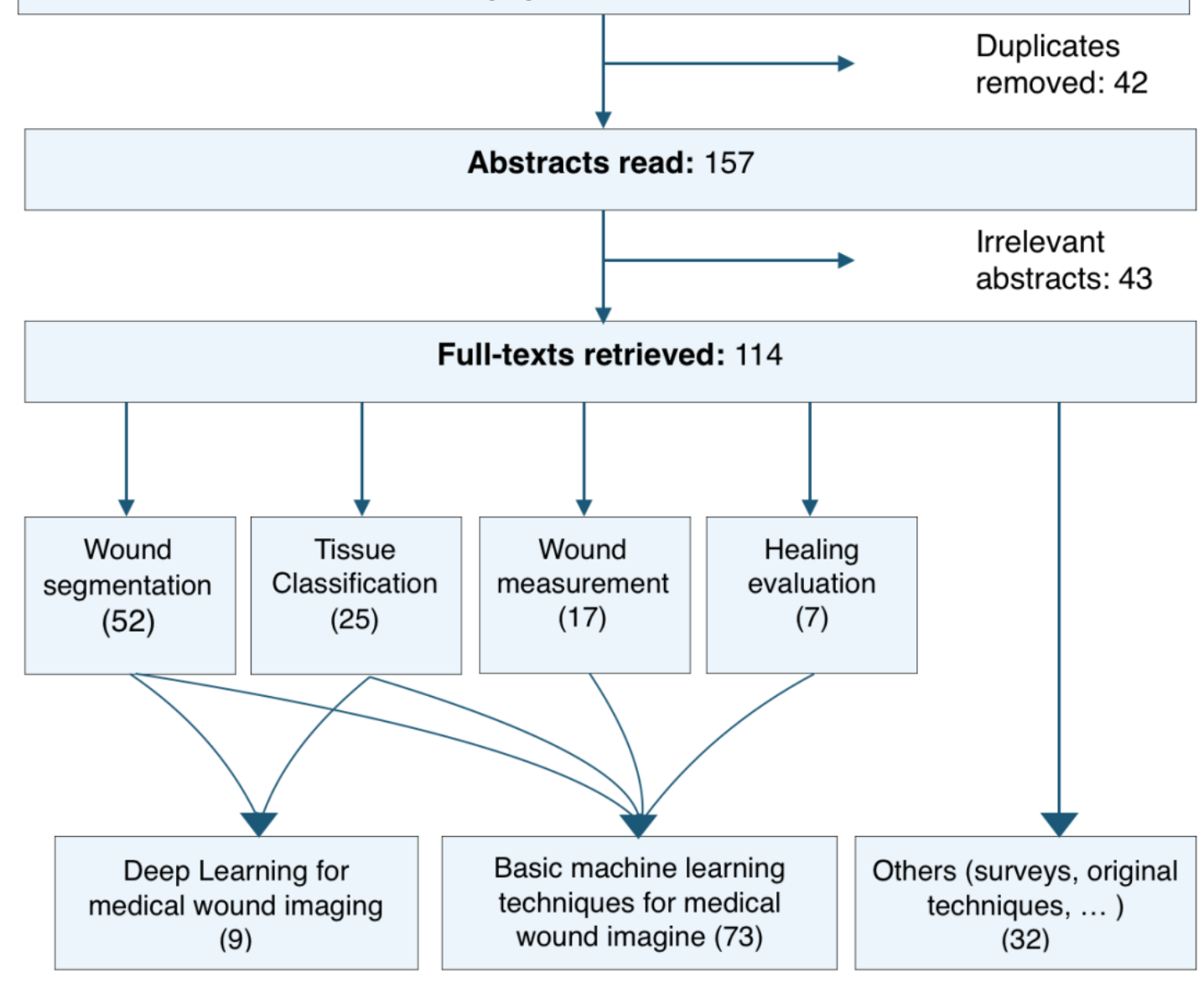

Figure 14: Study selection flow diagram.

analysis

After selecting the papers judged to be suitable for the review, 82 papers met the required criteria, and the corresponding full-texts were analyzed. Hence, the following information was extracted:

- Year and country: The studied topic has gained interest from re- searchers in the last decades. Hence, knowing its year of publication and the main country of affiliation 
is important to analyze the geographic regions dealing with this topic and when this interest has risen. The map in Fig. 15 represents the distribution of wound imaging researches by countries, where India has the highest number of contributions to this topic.

- The type of the publication: Type I: International journals, Type II: International conferences and workshops, and Type III: Book chapter. A fraction of $43 \%$ of the analyzed papers were journal papers and $56 \%$ were conference papers.

- Image database: The types of skin wounds studied were extracted in order to differentiate between the contributions to skin wounds imaging analysis. Furthermore, the number of images used re- presents an important element which enables to determine the efficiency of the proposed system since the majority of the techniques used are based on machine learning techniques that require a large set of images for the learning phase. 99\% of the papers used visible light images, whereas Chaves et al. (Chaves 2015) used thermograms and Bochko et al. (Bochko 2010) used near-infrared (NIR) images.
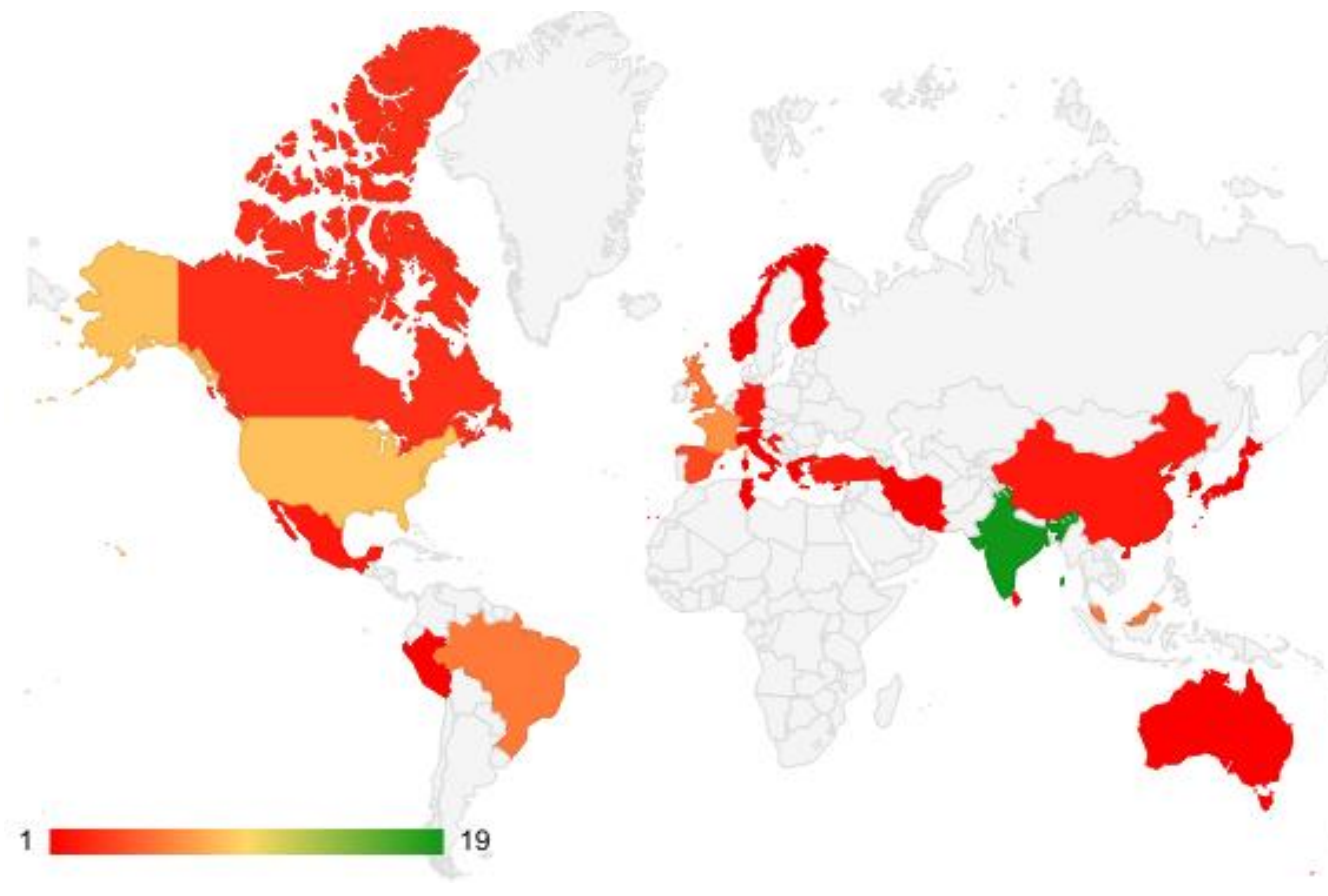

Figure 15: The analyzed articles in this review per country. 
- Image processing techniques: The assessment of skin wounds, as it will be presented in the following sections, can be done using several methods, depending on desired output (segmentation or classification or measurement, etc.). The diagrams presented in Fig. 16 and Fig. 17 present the main image processing techniques analyzed in the reviewed papers for wound segmentation and wound/tissue classification respectively. Generally, the preprocessing step is important in order to perform wound segmentation. It consists of noise reduction and filtering as well as color correction. The color space conversion is typically used in order to better distinguish between the wound and the skin. The most commonly used color spaces are: RGB, HIS and Ydb Dr color spaces. As for the segmentation step, different techniques have been used and the given results were promising. Since the databases used differ from one paper to another, as well as the number of images used, a comparison between the techniques could be misleading. The most common methods found in the analyzed papers, as presented in Fig. 15 were classification machine learning techniques (fuzzy C-means clustering (Chakraborty 2016)(Chakraborty 2015)(Trabelsi 2013),(Yadav 2013), K-means clustering (Veredas 2015),(Dalya 2016),(Haider 2016),(Chakraborty 2015),(Trabelsi 2013), (Hettiarachchi 2013),(Yadav 2013) for unsupervised classification, and support vector machines (Bochko 2010),(Dalya 2016),(Seixas 2015),(Kolesnik 2006),(Wang 2017) for a supervised classification). Once the segmentation is performed, a post-processing step is sometimes added in order to enhance the segmentation results such as morphological operations (Dhane 2016),(Dhane 2017) and filtering (Bochko 2010). As for wound/tissue classification techniques, support vector machines (SVM) was mainly used in the case of supervised classification (Veredas 2015),(Joseph 2016),(Mukherjee 2016),(Wannous 2011),(Wannous 2008),(Kosmopoulos 
2007), and K-means clustering for unsupervised classification (Hani 2012),(Wang

\section{5),(Hani 2012),(Hani 2011),(Wannous 2011).}

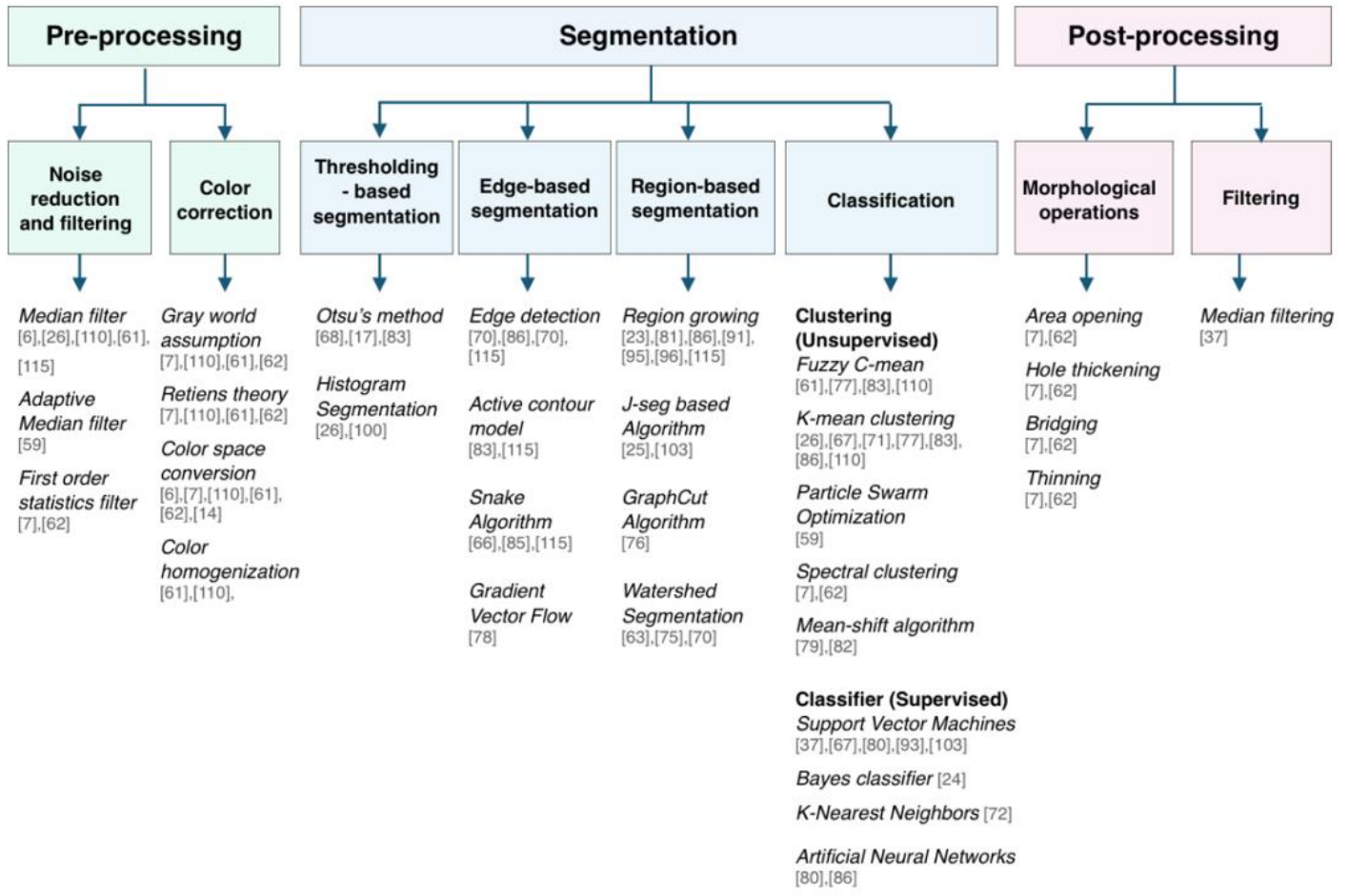

Figure 16: The analyzed papers per wound segmentation image processing technique. (Please refer to (Zahia 2020b) for the corresponding referenced papers)

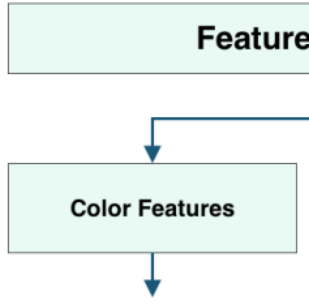

Color histogram
$[13],[26]$
Mean
$[97],[26],[14],[25]$
Skewness
$[97],[14]$
Standard deviation
$[97],[26],[14]$
Kurtosis
$[97],[14]$
Variance
$[97],[14]$
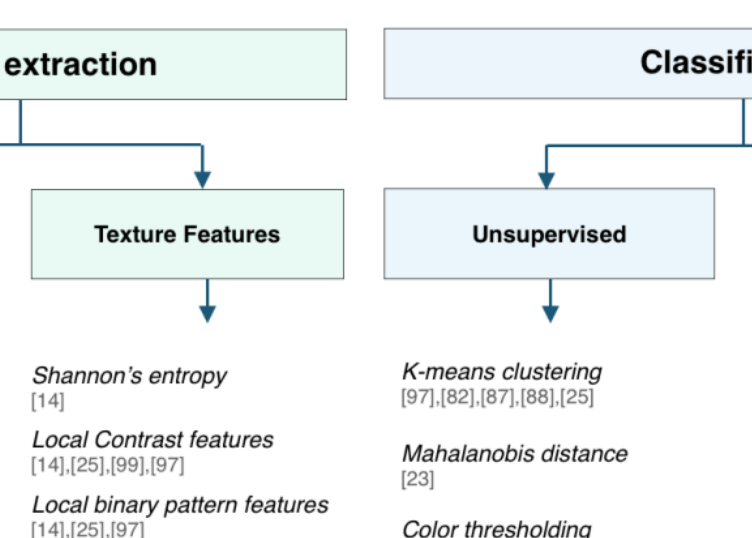

[14],[25],[97]

Gabor based features (GAB)

[25],[13]

Co-occurence matrix [25]

2-D Fast Fourier Transform

[68]

2-D Discrete Cosine

Transform

[68]

Topological and morphological features

[26]

Figure 17: The analyzed papers per wound/tissue classification image processing technique. (Please refer to

\section{K-means clustering Linear Discriminant Analysis $[97],[82],[87],[88],[25]$ \\ Mahalanobis distance [23] \\ Color thresholding [6] \\ Support Vector Machines [26],[68],[14],[25],[90],[13] \\ Bayesian classifier \\ [14] \\ Bayesian Committee Machine [23],[91]}

Neural Networks [26],[23]

Random Forest [26],

K-Nearest Neighbors [25]

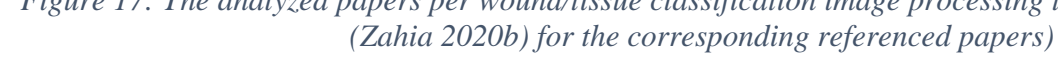


- Results: The main results metrics used in wound imaging validation are the accuracy, specificity and precision.

An optimal imaging system for pressure injuries assessment should verify all following criteria:

- An efficient detection of the wound whatever the clinical capturing conditions are: lightning, angle and distance.

- An accurate distinction between the wound and the rest of the image, with all possible objects included in the image such as: bed, bed sheet, a ruler or color panel and a person's hand or arm.

- A reliable measurement of the wound's characteristics such as: length, width, area and depth.

- Eventually, an optimal healing prediction with all the previous data given to the system.

In the literature, four main problems are addressed in pressure injury image analysis, as depicted in Fig. 14: Segmentation, measurement, tissue classification and healing evaluation.

\subsubsection{Segmentation}

Finding the boundaries of a pressure injury in an image is typically a first necessary step that allows subsequent analyses. In the past, medical staff used computer pointing devices to delineate the wound boundary in pressure injury images (Delode 2001). However, this manual approach was unreliable due to the subjective component involved 
in the process (e.g. differences of opinion between clinicians about the actual limits of the wound) (Bulstrode 1986). For this reason, automated segmentation tools have been developed to perform objective ulcer segmentation.

One of the first techniques used for ulcer segmentation were edge- based segmentation techniques, such as active contour models (ACM) or Snakes algorithm (Silveira 2009),(Hettiarachchi 2013),(Jones 2000). For instance, Jones and Plassmann (Jones 2000) presented ACM that used piecewise B-spline arcs and the minimax principle to adaptively regularize the ulcer contour according to the local conditions in the image, using wound contour manual delineations to initialize the ACM. Their approach significantly reduced the inherent variations upon repeatability and consistency of ulcer area measurements.

However, most approaches to pressure injury segmentation have been based on similarity-based segmentation techniques. This is the case of the statistical color models by Veredas et al. (Veredas 2015b), who used a set of training images to build color histogram models for four different wound tissue types. Then, using a Bayesian approach, they computed the posterior membership probability of the pixels of an incoming pressure injury image, thus assigning each pixel to the most likely type of tissue type and obtaining a segmentation of the ulcer.

The main disadvantage of supervised approaches is the need for either (i) a large enough set of training images to learn significant features for ulcer segmentation or (ii) the manual annotations by an expert, which has an impact in terms of cost, precision and reliability. For this reason, unsupervised approaches constitute an interesting alternative. 
An example of this is the use of spectral clustering for ulcer segmentation presented in (Dhane 2016). In said work, the authors first preprocessed the images, performing color homogenization by combining the gray world assumption and Retinex theories, reducing noise via first order nonlinear filters, and converting the images to the HSV color space to improve image quality for accurate segmentation of the wound area. Then, spectral clustering was applied to find homogeneous regions in the image, resulting in segmented regions which were post-processed using the opening, hole filling, thickening, bridging and thinning morphological operators to obtain the desired segmented image. Then in their next work (Dhane 2017), they used calculated mean contrast to select the best color channel from 14 color spaces. They found that the $\mathrm{Db}$ channel had the highest contrast and enabled better segmentation results compared to the others. Another contribution of the new approach was to use gray based fuzzy similarity measure and segment the resulting gray image using spectral clustering. The same methods of postprocessing presented in their previous system [7] were applied in this work in order to retrieve better segmentation results.

Another proposal for unsupervised pressure injury segmentation was recently presented by Ortiz et al. (Ortiz 2017). The proposed method searched for contrast changes in the image, extracting synthetic frequencies using a model of the energy density over an electric field to describe a relation between a constant density and the pixels intensity. Furthermore, a toroidal geometry was used to decompose the image over multiple contrast levels by varying the synthetic frequencies, and finally the decomposed image was segmented by applying Otsu's method (Otsu 1979) to obtain the contours corresponding to contrast changes in the image. The same authors integrated this method afterwards in a 
mobile application in order to enable nurses to assess the captured pressure injuries in real time (Garcia-Zapirain 2018). Chakraborty et al. (Chakraborty 2016) presented a wound segmentation technique using preprocessing followed by fuzzy K-means clustering. The results showed a very high segmentation accuracy $(98.98 \%)$. Then the same authors presented in (Chakraborty 2017) an approach for chronic wound segmentation using preprocessing techniques such as noise reduction and filtering followed by particle swarm optimization (PSO). This method is a stochastic global optimization algorithm which was based on swarm intelligence (Bonabeau 1999) and characterized with a fast convergence rate.

Another approach for wound segmentation was based on machine learning methods. Bachko et al. (Bochko 2010) proposed a system of lower extremity ulcers segmentation and measurement using support vector machine classifier. In fact, the studied images were acquired using a developed four-band spectral digital camera with captured near-infrared (NIR) images. These images were first preprocessed by normalizing them and deleting all irrelevant information on the edges of the images. Then SVM classification was applied after a training step using 18 images. Finally, a post-processing step consisting of smoothing the images using Median filtering was applied on segmented images.

Fig. 18 depicts some pressure injury image segmentation results, extracted from a selection of papers in the literature. 


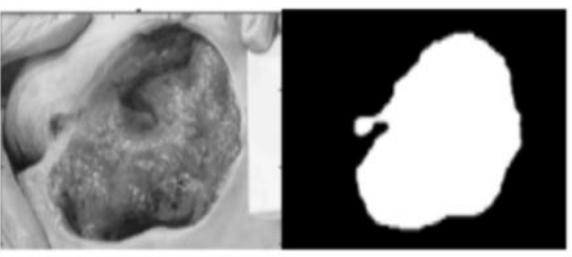

(a)

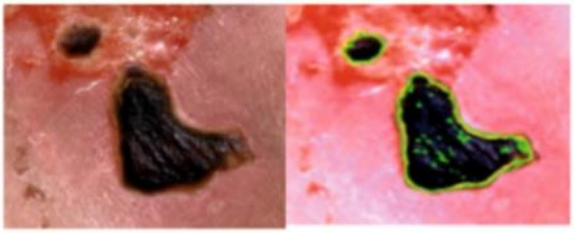

(c)

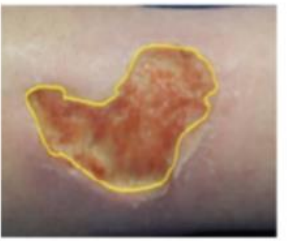

(e)

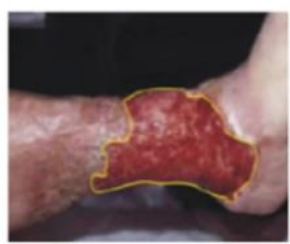

(f)

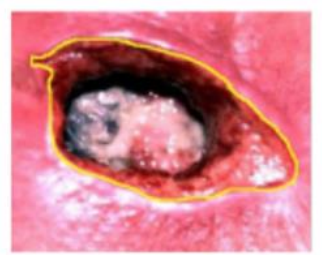

(b)

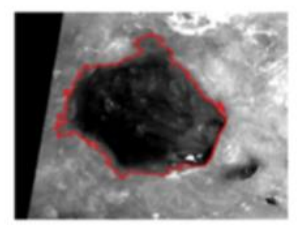

(d)

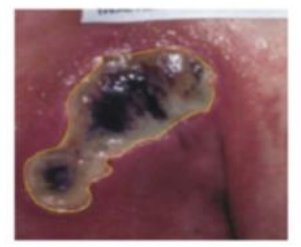

(g)

Figure 18: Collage of some pressure injuries segmentation results:

(a) Pressure injury segmentation using energy density model for contour extraction proposed by Ortiz et al (Ortiz 2017). (b,c) Wound segmentation using spectral clustering and morphological post processing, proposed by Dhane et al. (Dhane 2016) (d) Wound segmentation using spectroscopy and 3D stereophotogrammetry proposed by Paluchowski et al. (Paluchowski 2013). (e-g) Wound segmentation using fuzzy based thresholding and mathematical morphology operations, proposed by Mukherjee et al. (Mukherjee 2014)

\subsubsection{Measurement}

The physical parameters of a pressure injury (e.g. its area, depth, volume) constitute a valuable clinical indicator for assessing the progress of healing. This is the reason why the literature presents several works devoted to the automatic measurement of such parameters from images, using mainly 3D imaging techniques.

One of the earliest approaches to measuring the area and volume of pressure injuries was MAVIS (Measurement of Area and Volume Instrument System) (Plassmann 1998). Projecting structured light (i.e. parallel stripes of alternating colors) on the region of interest, and using a standard camera to capture the image, the authors made it possible to 
generate a three dimensional map of the ulcer. Using cubic spline interpolation to simulate the healthy skin surface, the volume of the ulcer could be computed.

The use of different image modalities was the main novelty of the multimodal vision system combining 2D imaging spectroscopy and 3D stereo-photogrammetry presented in (Paluchowski 2013). The proposed system was formed by a high resolution hyperspectral camera and a monochromatic video frame camera mounted on the same scanning system. The acquisition of stereo images provided information about the ulcer surface geometry, while the hyperspectral image was employed to detect the wound boundaries using spectroscopic analysis. Finally, creating a surface model of the region surrounding the ulcer allowed reconstructing the top surface above the wound and thus, to compute its volume.

However, these approaches require using costly and complex lightning and imaging equipment, thus hindering its application by non-trained medical staff or its widespread use in the first line of health care. For this reason, Albouy et al. (Albouy 2007) addressed the problem of creating 3D models of the ulcer by matching two uncalibrated images captured with different angles.

The same authors extended their work in (Treuillet 2009), designing an iterative matching scheme to generate a dense estimation of the surface geometry from two widely separated uncalibrated images taken with a hand-held digital camera with free zooming, obtaining accurate volumetric measurements.

The same approach was proposed by Yee et al. (Yee 2017) using iDr app. This app was designed to accurately and non-invasively reconstruct 3D wound model and measure the wound's area and volume in 3D digital space. When a video of the wound is taken, the wound is imaged from different viewpoints. Then, structure from motion (SFM) algorithms 
select image pairs that will be used to reconstruct the wound by the mean of stereoscopic $3 \mathrm{D}$ reconstruction techniques. The accuracy of this app was measured by comparing the retrieved metrics using iDr with a commercial structured light based 3D industrial camera which has an accuracy level of $50 \mu \mathrm{m}$. The iDr app yielded an overall average relative error of $1.66 \%$ for distance measurement, $1.14 \%$ for area measurement and $4.41 \%$ for volume measurement.

The quantitative characteristics of pressure injuries were also measured using the detection of a ruler which is generally placed next to the wound while taking the photographs. The ruler often includes a color panel which permits the color correction, the conversion from pixel size to real size, and the detection of the angle from which the images were taken using computer vision stereoscopy methods. Li et al. (Li 2017) proposed an approach for wound surface, area, length and width measurement. Using 32 pressure injury images containing a ruler with 4 color panel, they first applied an RGB to YCbCr color conversion and detected the skin using Gaussian model. An SVM classifier was then used to distinguish between pixels belonging to the wound and the others belonging to healthy skin. Once the wound was segmented, the authors used the detected color panel to adjust the image's perspective and retrieve the real dimensions using pixel to real distance conversion.

\subsubsection{Tissue classification}

As the severity of pressure injuries worsen, subcutaneous tissues become visible. For this reason, it is interesting to automatically distinguish the types of visible wound tissues, as it constitutes a relevant indicator of the ulcer severity status. 
In an early work, Delode et al. (Delode 2001) designed an image processing algorithm to detect and measure the area of different types of ulcer tissues. First, the authors extracted the wound boundary from the image. Then, median filtering was applied to remove noise, and finally they obtained a binary mask corresponding to the wound region, which allowed computing its area. Finally, to distinguish tissues, the authors simply performed a color model conversion from RGB to HSV, which allowed to detect the number of black, red and yellow pixels inside the wound region, which correspond to different types of tissues.

More recently, tissue classification techniques have relied on extracting visual features from the different types of tissues of interest, and training supervised classifiers to detect them. For instance, the work by Kosmopoulos and Tzevelekou (Kosmopoulos 2007) presented an approach that aimed at determining the evolution stage of segments of the pressure injury. To that end, they used a supervised machine learning approach based on a support vector machine (SVM) classifier. First, they gathered a set of training pressure injury images that were automatically segmented into homogeneous regions using graphbased techniques (Felzenszwalb 2004). Then, each image region was presented to an expert user, who categorized it into one of the four pressure injury severity stages defined by the European Pressure Ulcer Advisory Panel (EPUAP 1999). Next, the assigned labels, together with color (HSV histograms) and texture (Gabor wavelets) features of each region were used to train the SVM. Finally, this classifier was used to categorize the regions of an incoming pressure injury image into one of the four severity stages. Also Veredas et al. (Veredas 2015) carry out tissue type classification using three different machine learning methods namely: neural networks, support vector machine and random-forest decision 
trees and the results show that the highest rates are given by SVM and random-forest models.

In (Veredas 2010), the authors presented an approach combining neural net- works and Bayesian classifiers for automatic tissue identification. As a first step, the wound region was segmented using an adaptive mean shift procedure and the region growing algorithm. From the segmented region, color and texture feature vectors were extracted and fed to a set of multilayer perceptrons, which were trained to classify its inputs into categorical tissue classes determined by clinical experts. Finally, a Bayesian committee machine was formed by training a Bayesian classifier to combine the classifications of the neural networks, designing specific heuristics based on the wound topology to improve the tissue classification results. Chakraborty et al.(Chakraborty 2017) presents in the same paper discussed in section II chronic wound tissue classification using linear discriminant analysis (LDA) in order to distinguish the wound tissues (granulation, slough, and necrotic tissue). The same author presented in (Chakraborty 2016) wound tissue classification using LDA but the segmentation method was different from his next paper (Chakraborty 2017). They proved that LDA method outperformed the other supervised state-of-the-art (K-NN, fuzzy K-NN, K-means, SVM and Bayesian) methods with an overall classifi- cation accuracy reaching $91.45 \%$.

The work by Wannous et al. (Wannous 2011) presented a single view and a multiview approach to tissue classification. As for the single view ap- proach, the general idea resembled the work in (Veredas 2010) in several ways: first, the wound was segmented in an automatic manner using un- supervised segmentation algorithms efficient on textured images. Then, color descriptors (such as the mean color descriptor, the dominant color 
descriptor, and 2D and 3D color histograms) and texture descriptors (like local binary patterns, or Gabor features) were extracted from the segmented region.

And next, an SVM classifier was trained to classify the pixels of an incoming pressure injury image into one of the three tissue classes defined by clinicians (granulation, slough and necrosis) in a previous manual annotation process. In the multiview approach, a 3D model of the wound was obtained using uncalibrated vision techniques upon two images of the wound, and each triangle of the 3D mesh was assigned the most likely tissue type label by applying a merging algorithm.

As in the previous works, the pipeline formed by (i) wound segmentation, (ii) color and texture feature extraction and (iii) supervised classification, was also adopted to design the architecture of the system presented in (Mukherjee 2014). In that work, the authors employed fuzzy divergence-based thresholding for segmenting the wound region. Next, statistical color descriptors computed upon 15 different color spaces were extracted, and texture feature vectors like Shannon's entropy, contrast features or local binary patterns computed on the aforementioned color spaces were also created. With these features, SVM and Bayesian classifiers were trained to classify pixels as corresponding to granulation, slough and necrotic tissues. Figure 19 depicts some pressure injury tissue classification results in the literature. 


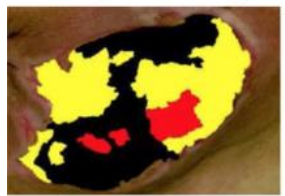

(a)

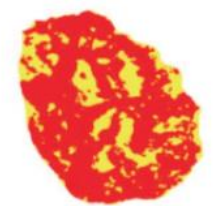

(d)

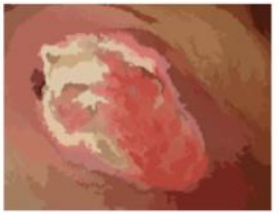

(g)

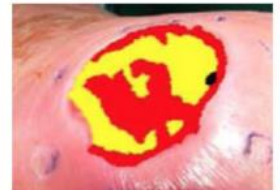

(b)

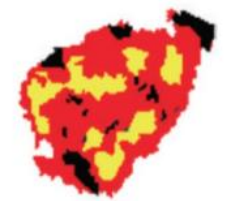

(e)

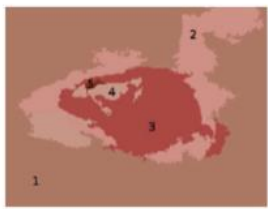

(h)

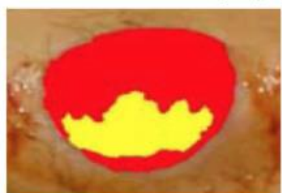

(c)

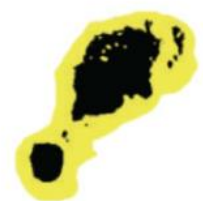

(f)

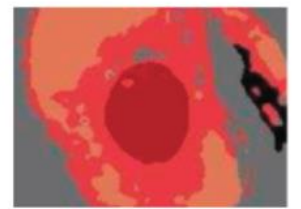

(i)

Figure 19: Collage of some pressure injuries Classification results. (a-c) wound tissue classification using SVM, proposed by Wannous et al. (Wannous 2011). (d-f) Wound tissue classification using SVM, proposed by Mukherjee et al. (Mukherjee 2014). (g,h) Pressure injury tissue classification using neural networks and Bayesian classifiers, pro- posed by Veredas et al. (Veredas 2010). (i) Lower extremity ulcer image classification using SVM and post-processing, proposed by Bochko et al. (Bochko 2010).

\subsubsection{Healing evaluation}

Coupling the quantitative parameters extracted from pressure injury image analysis with medical domain knowledge allows evaluating the healing process of the wound, thus bridging the gap between image analysis and pressure injury diagnosis. In (Bon 2000), Bon et al. defined a healing function based on monitoring time-dependent statistical image parameters related to luminance, color, and homogeneity, as well as the number of luminance minima and maxima inside the ulcer region. Using analysis of variance, the authors were able to identify those parameters that changed during healing, and thus were clinically relevant. Other authors have addressed the same topic by using thermal images. Based on the fact that wound temperature can provide quantitative data for monitoring the healing process, Chaves et al. (Chaves 2015) took thermograms of 
patients suffering from sacral pressure injuries subjected to two different medical treatments for wound healing. The authors observed that the ulcers of those patients following the treatment healed within a 4 weeks period, and most importantly, the healing correlated with the ulcer temperature observed in the thermograms, thus proposing thermography as an ancillary technique for pressure injury healing evaluation.

Applications for wound assessment have also been developed, in order to analyze the images and retrieve the information which will be useful for the evaluation of the wound's healing. Some of them have showed good results but most of the time the assessment is not totally automatic. Filko et al. (Filko 2010) presented an application for wound analysis and management named WITA: wound image based tissue analysis. It achieves wound tissue classification and healing evaluation through wound digital image processing. The analyzed input images are manually segmented by the user and then the system operates the tissue classification using statistical pattern recognition algorithm (which were not specified in the paper) and wound measurement (area, circumference, width and height) but the user needs to precisely define the distance from which the ulcer was captured. WITA also depicts the healing evaluation after more than two time-spaced images of the same patient.

In (Noguchi 2014), Noguchi et al. presented the application of image processing to wound blotting images obtained by applying molecular cytology techniques to pressure injury healing evaluation. Those images showed a stained version of the wound that revealed micro-level mechanisms occurring during the healing process. To evaluate the healing process, the authors proposed a pipeline consisting in (i) dividing the image in windows, (ii) extracting texture features like gray level co-occurrence matrix, wavelets, 
and local binary patterns from each window, (iii) applying K-means clustering or SVM classification to categorize each window into one of three types of patterns helpful for wound assessment: dot, cloud and plain.

Another method for wound healing evaluation consists of tracking the evolution of wound metrics such as: the diameter, area and volume. 3D reconstruction techniques are used to retrieve the $3 \mathrm{D}$ representation of the wound in order to measure the main aforestated characteristics. For instance, Yee et al. (Yee 2017) used iDr app (as presented in section III-B) to track the healing of rat wounds through a period of 3-weeks.

\subsection{Chapter Summary}

In this literature review chapter, we have given an overview on Deep Learning architectures and biomedical imaging modalities used in this field of research. We have shown through the latest research achievements that Deep Learning has become the basis

of numerous emerging applications in biomedical imaging. We have also given a detailed state of the art on the pressure injury imaging techniques which have been previously addressed using image processing and classical machine learning techniques.

In the following chapters, we will introduce the proposed frameworks for tissue types segmentation and classification, pressure injury segmentation and measurement, where we were able to automatically retrieve: the volume, depth, area, major and minor axes, and finally the integration of the segmentation pipelines in a web-based application. 


\section{CHAPTER 3}

\section{PRESSURE INJURY TISSUE}

\section{CLASSIFICATION}

\subsection{Introduction}

This chapter presents our proposed approach for the classification of pressure injury tissue types. This task was handled in the early stage of the Ph.D, while the dataset was being acquired from hospitals. To enable a better diagnosis of pressure injuries, this research step addresses the detection of the presence of either the granulation tissue, slough and/or necrosis in the wound by designing an end-to-end tissue classification framework based on a Convolutional Neural Network model (Zahia 2018). This method enables a semantic segmentation of the tissue types inside the wound. A preprocessing step removes the flash light and creates a set of $5 \times 5$ sub-images which are used as input for the CNN network. The network output classifies every sub-image of the validation set into one of the three 
classes studied. The metrics used to evaluate our approach show an overall average classification accuracy of $92.01 \%$, an average total weighted Dice Similarity Coefficient of $91.38 \%$, and an average precision per class of $97.31 \%$ for granulation tissue, $96.59 \%$ for necrotic tissue, and $77.90 \%$ for slough tissue.

The rest of this chapter is organized as follows: a description of the dataset is presented in section 3.2. Section 3.3 overviews the proposed framework by presenting data preprocessing procedure and the $\mathrm{CNN}$ model chosen for the classification and section 3.4 discusses the experimental results and analysis.

\subsection{Dataset}

The dataset is made up of 22 images of stage III and IV pressure injuries for training and testing, acquired from the Igurko Hospital, Bilbao-Spain, with 4 being purchased from The National Pressure Ulcer Advisory Panel (NPUAP) online store for validation purposes (NPUAP store). The training and test images have a resolution of $1020 \times 1020$ and were taken using flash due to poor illumination in the nursing facility. The wounds in this dataset are infected, contain necrotic tissue, or are in a healing state evidencing granulation tissue, and all the combinations from tissue types are present in the dataset. These images were automatically cropped in 270,762 RGB matrixes of $5 \times 5 \times 3$ in size for granulation tissue, 37,146 for necrotic tissue, and 80,636 for slough.

The images were manually segmented using expert knowledge from medical personnel in order to obtain the ground-truth labeling for tissue classification. A control group comprising two physicians was told to independently examine the ground-truth images and document their professional criteria regarding our manual segmentation. The final 
segmentation was then obtained by merging both the concept from the control group and the originally proposed marked segments. The four segments selected were labeled as follows: The external skin is represented by a black background (graylevel 0), necrosis by dark gray (graylevel 89), the granulation by light gray (graylevel 170) and slough by white (graylevel 255). The Fig. 20 shows a set of eight original images (1st and 3rd row) and their corresponding ground-truth (2nd and 4th row). Once the dataset was created, it was divided into 2 parts: training set and testing set. The partition percentage would be around $75 \%$ for the training set, and $25 \%$ for the testing set as shown in the following table:

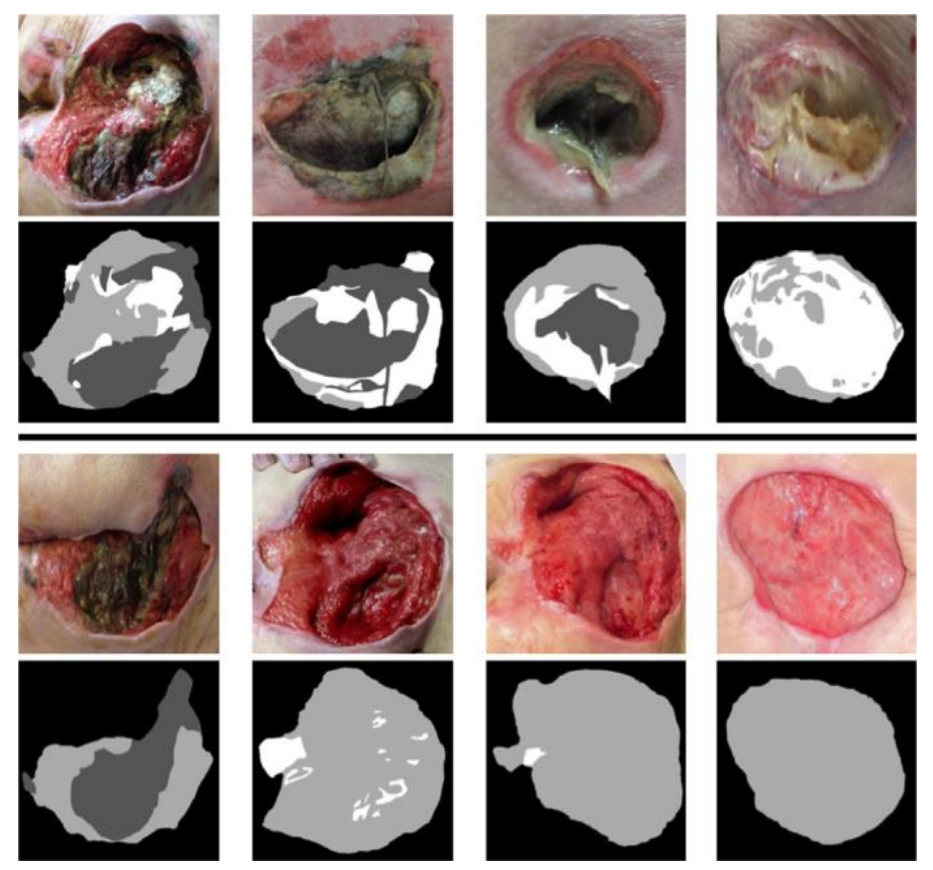

Figure 20: A set of pressure injury images and their corresponding ground-truth segmentation used to create the dataset and labels

Table 1: Number of images for the training and testing of CNN architecture

\begin{tabular}{c|cc} 
& Training & Testing \\
\hline Granulation & 203,072 & 67,690 \\
Necrosis & 27,860 & 9286 \\
Slough & 60,477 & 20,159 \\
\hline
\end{tabular}




\subsection{Proposed framework}

Unlike the state of the art methods that tend to use a large number of images to train the network to obtain a good segmentation results, our approach involves using a limited number of high resolution images and extracting a larger dataset of small images in order to achieve comparable segmentation of the pressure injury. Our architecture for image segmentation is comprises a preprocessing step which from a $1020 \times 1020$ original pressure injury image crops $5 \times 5$ images of the different tissue types as output images with their corresponding labels. The choice of small patch size was based on the size limit in which we do not lose the textures belonging to each class, and the average number of pixels defining the boundary between two classes in the images studied. Once the Convolutional Neural Network is trained, the segmentation of the validation image is then carried out by partitioning the image into $5 \times 5$ blocks and classifying each one of them according to the class predicted, from which we construct the segmented image. Figure 21 sketches our proposed workflow for tissue types segmentation and classification.

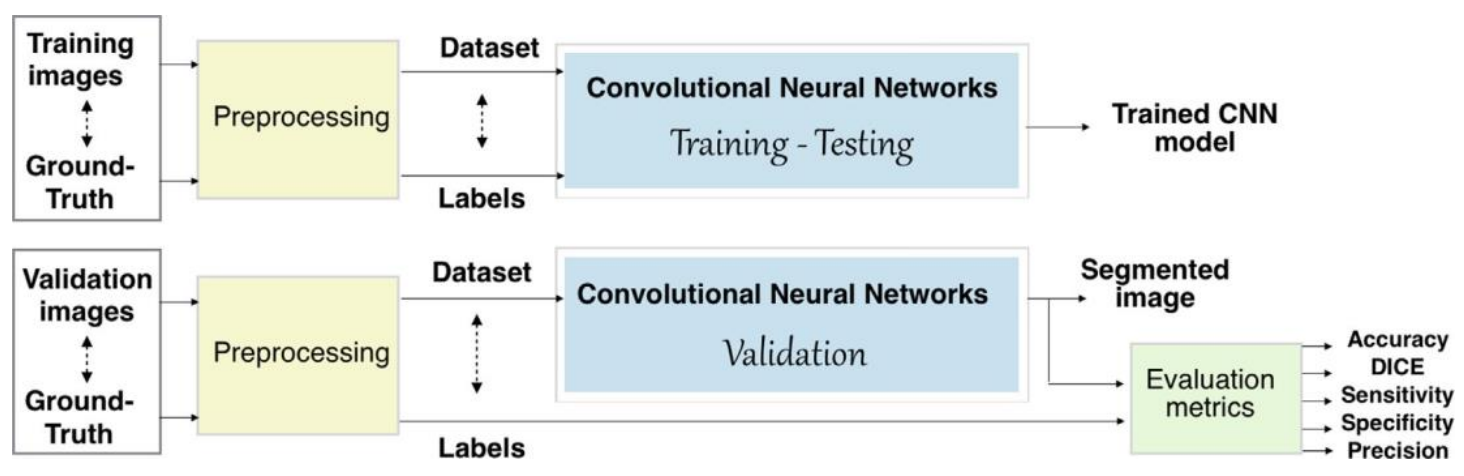

Figure 21: Proposed architecture for image segmentation using Deep Learning technique 


\subsubsection{Preprocessing}

The first block in the training step for our system aims at creating the dataset which will be given as input images and their labels to the CNN architecture. Since our focus is on tissue classification within the wound area we do not address automatic image masking as presented in (Ortiz 2017),(Rickard 2003), and a black mask was applied on original pressure injury images in order to retain only the wound area. From the extracted region of interest, we then need to remove flash light reflection. To achieve this, we convert the images into grayscale in accordance with the radiometric equation (Bovik 2010):

$$
\mathrm{Y}=0.2126 . \mathrm{R}+0.7152 . \mathrm{G}+0.0722 . \mathrm{B}
$$

These images are then turned into binary images using Otsu's Method described in (Raja 2014) where in order to define the white areas.

$$
\begin{aligned}
& \mu_{0}=\sum_{i=0}^{t-1} \frac{i p_{i}}{\omega_{0}(\mathrm{t})} \\
& \mu_{1}=\sum_{i=t}^{t-1} \frac{i p_{i}}{\omega_{1}(\mathrm{t})}
\end{aligned}
$$

where $\mu_{0}(\mathrm{t})=\sum_{i=0}^{t-1} i p_{i}$ and $p_{i}$ is the probability for the gray scale level $i$. The mean intensity $\mu_{T}$ is represented as $\mu_{\mathrm{T}}=\omega_{0} \mu_{0}+\omega_{1} \mu_{1}, \omega_{0}+\mu_{1}=1$. The objective is to find $t$ that maximizes

$$
\mathrm{J}(\mathrm{t})=\sigma_{0}+\sigma_{1}
$$

where $\sigma_{0}=\omega_{0}\left(\mu_{0}-\mu_{\mathrm{T}}\right)^{2}$ and $\sigma_{1}=\omega_{1}\left(\mu_{1}-\mu_{\mathrm{T}}\right)^{2}$. By using the threshold $\mathrm{t}=0.95$, each pixel is separated into two classes depending on the amplitude in gray scale values. Once the binary image has been obtained and flash light zones detected, we then dilate the binary images in order to enlarge these zones. The corresponding zones in the original images are then filled with the value of their boundary pixels in order to retrieve the color of the tissue hidden under the flash light. Once the flash light artifacts 
had been removed, the ground truth was cut into $5 \times 5$ images in order to have a larger amount of sub-images, which each one containing only one class. Our database contains 22 of $1020 \times 1020$ images and by applying this technique, we ended up with more than 380,000 small images each one containing a part of one of the studied tissue types. This technique represents the proposed solution to enlarge the database. As mentioned previously, the choice of patch size was made for 2 reasons: a smaller size $(1 \times 1$ for example) would not preserve the textures and would lead to a confusion between the classes subject to study. In addition, the average number of pixels defining a boundary between two classes in the studied images was measured manually and resulted in 5.48 pixels, hence the size $5 \times 5$ chosen. Once we know the label for each one of the patches extracted, we then select the corresponding sub-images in the original images and save them in separate files for the different classes we have at our disposal. The figure below (Fig. 4) represents the preprocessing step in our system.

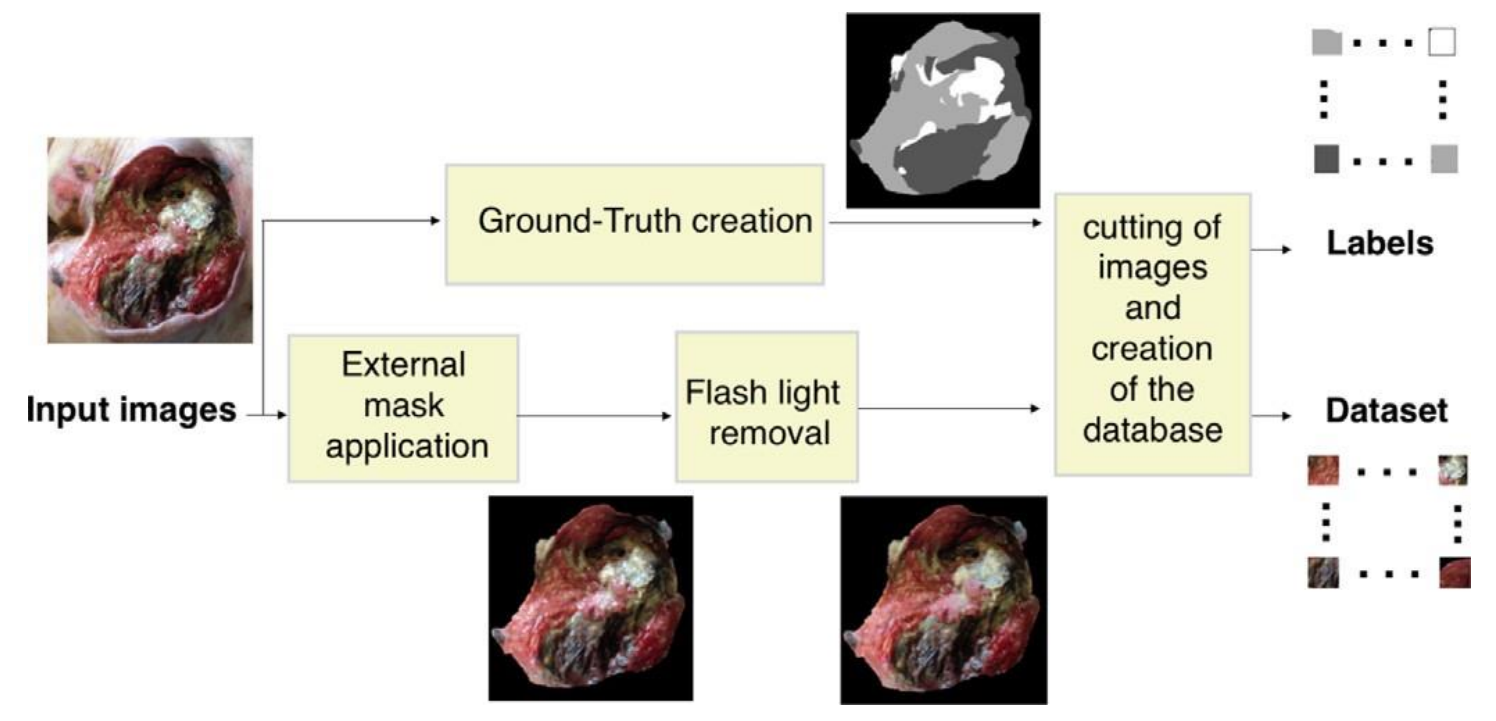

Figure 22: Preprocessing step for database creation

An example from the $5 \times 5$ matrices with tissue information is shown in Fig. 5 . Note that the matrices labeled as granulation tissue are similar to each other, although the 
necrotic and slough tissue sub-sets are different. This is due to the fact that necrotic tissue also includes shadows present in the pressure injuries and the slough tissue images have different colors and features because, depending on the bacteria producing the infection, the suppuration color changes.
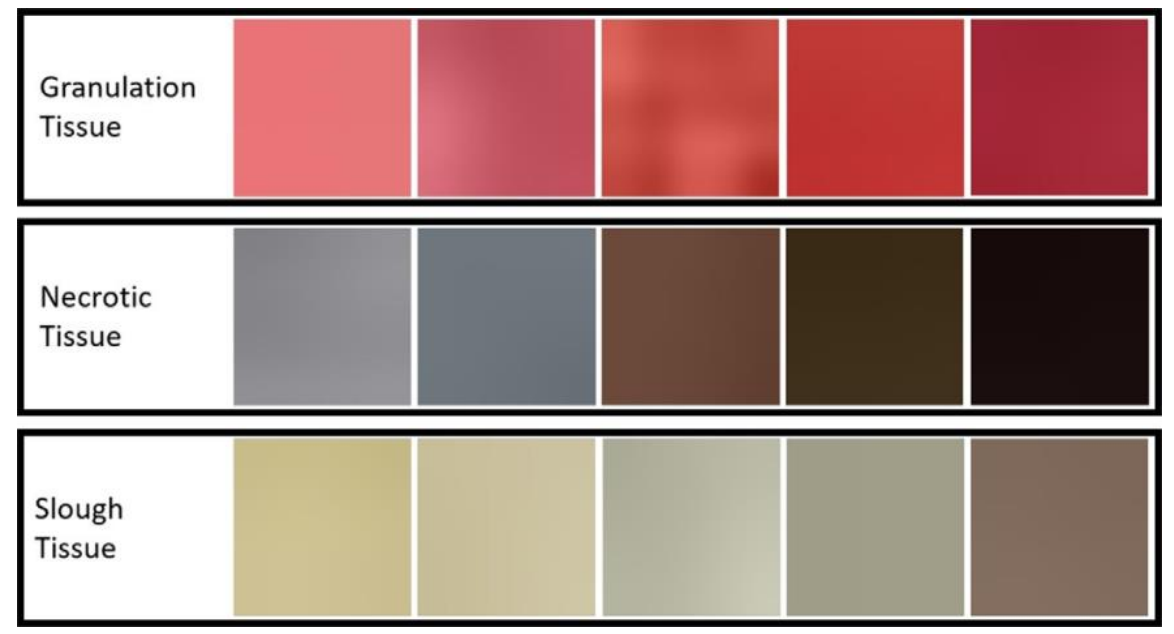

Figure 23: Dataset dictionary for the three different tissue types: Granulation, Necrotic

\subsubsection{Deep Learning model}

Our system is made up of 9 layers: 3 convolution layers where the first two are with zeropadding $(2 \times 2$ and $1 \times 1)$, and each one is followed by a Rectified Lineal Unit (ReLU1, ReLU2 and ReLU3). The number of feature maps and weights in each convolution layer is $10,20,30$ and $760,560,840$ respectively. Each feature map detects one kind of features across the image. The choice of filter size was made so as to preserve the textures that belong to each different tissue type in the pressure injury. The fully connected layer is then followed by a Softmax Layer, ending with a classification layer which gives rise to the probability of patch belonging to one of the 3 predicted classes, as shown in Fig. 23. The algorithm was coded on Matlab 2017a using Neural Network Toolbox. 


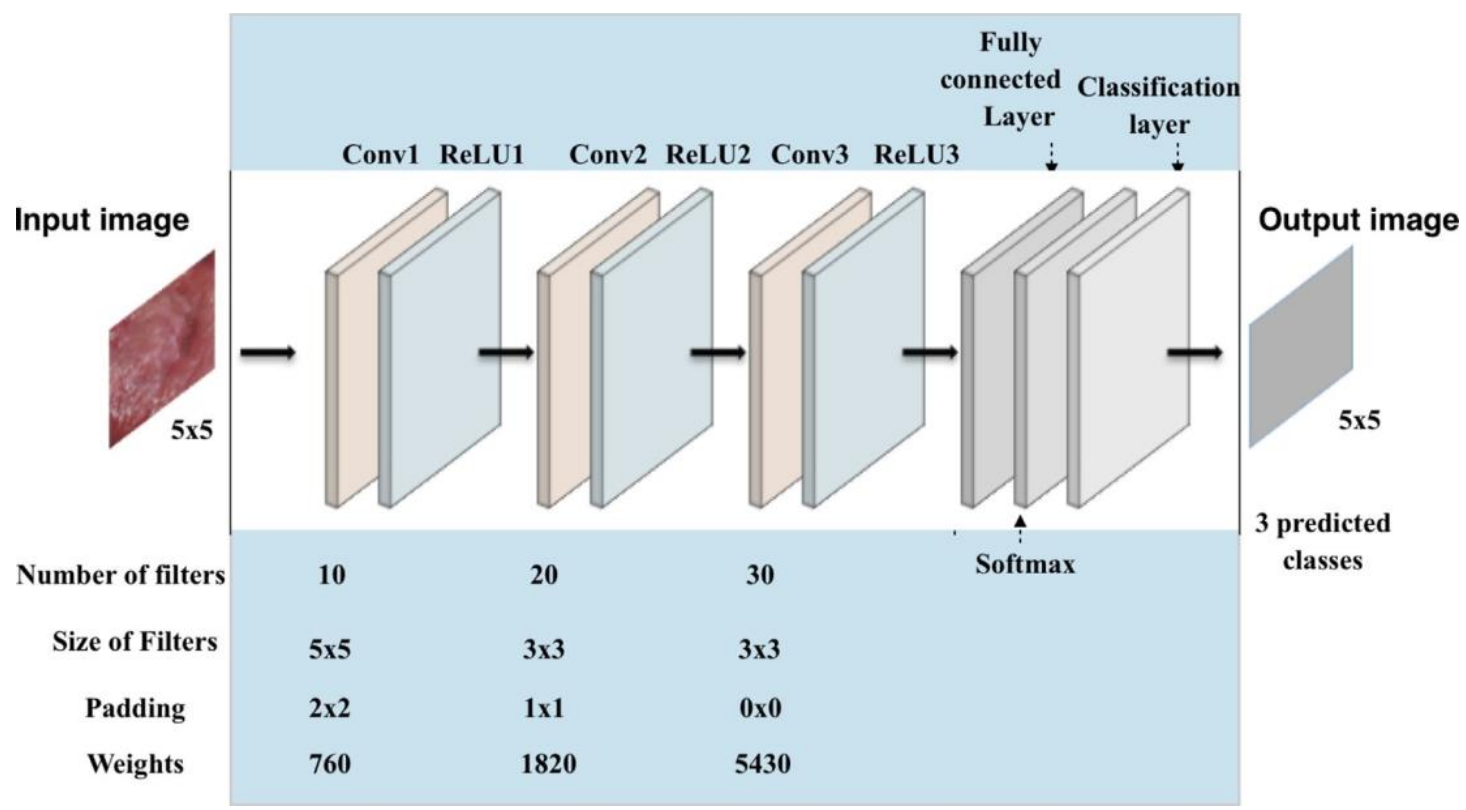

Figure 24: Proposed Convolutional Neural Network architecture for image segmentation.

\subsection{Results and Discussion}

\subsubsection{Validation metrics}

The segmentation method was evaluated using five performance measures: accuracy, Dice Similarity Coefficient (DSC), sensitivity, specificity and precision (Litjens 2017). To explain these metrics, the different regions that are generated when comparing the ground truth with the segmented results obtained are shown in Fig. 24.

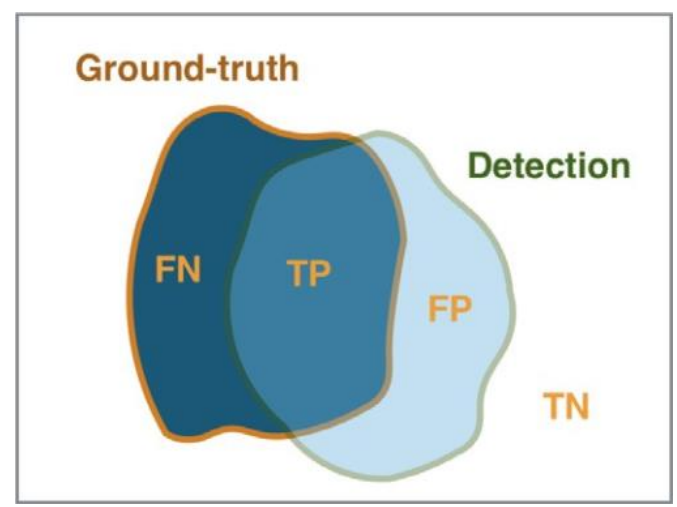

Figure 25: Illustration of different segmentation areas for performance metrics measurement. 
The True Positive (TP) region refers to those pixels that were correctly classified in a targeted class, the False Positive (FP) region refers to those pixels mistakenly classified as belonging to the targeted class and the False Negative (FN) region refers to those pixels belonging to the targeted class that were not classified in that class, while the True Negative (TN) region refers to those pixels that do not belong to the target class and are excluded.

- Accuracy: the segmentation accuracy is the ratio of the number of pixels accurately clustered by the algorithm out of the total number of pixels segmented.

- Dice Similarity Coefficient (DSC): this is measured for each class segmentation considered apart, and also for the whole segmentation, by calculating the sum of weighted dice similarity coefficients of each class as follows:

$$
\text { DSC }=\frac{2 \mid \text { Ground_truth } \cap \text { Detection } \mid}{\mid \text { Ground_truth }|+| \text { Detection } \mid}
$$

The weighted DSC for the whole segmentation is as follows:

$$
\mathrm{DSCw}=\sum_{i=1}^{n} D S C_{i} \times w_{i}
$$

where $\mathrm{DSC}_{\mathrm{i}}$ is the DSC corresponding to each class when measured alone, and $\mathrm{w}_{\mathrm{i}}$ is a ratio of the number of pixels in the class $i$ out of the sum of pixels of all the classes.

- Sensitivity: also named "recall", this represents the ratio of class pixels accurately segmented out of the union of ground-truth same class pixels.

$$
\text { Sensitivity }=\frac{\mathrm{TP}}{\mathrm{TP}+\mathrm{FN}}
$$

- Specificity: this represents the ratio of negatives that are correctly identified.

$$
\text { Specificity }=\frac{T N}{T N+F P}
$$

For exact segmentation sensitivity, specificity and total DSC should be equal to one.

- Precision: this represents the ratio of a class pixels accurately clustered out of the 
union of segmented same-class pixels.

$$
\text { Precision }=\frac{\text { TP }}{\text { TP+FP }}
$$

\subsubsection{Results}

The significant advantage of our proposed approach lies in its capability to accurately segment the wound's different tissue types. We measure the performance of our proposal for both the classification and the segmentation tasks, and the accuracy of the classification of the different 3 types of tissue was $92.01 \%$. Classification from these masks allows us to perform image segmentation in spite of the adverse registry conditions such as diverse illumination conditions and image distortion, among others. The results for the segmentation task are presented for the pressure injuries depicted in Fig. 25 are shown in Tables 1-5.

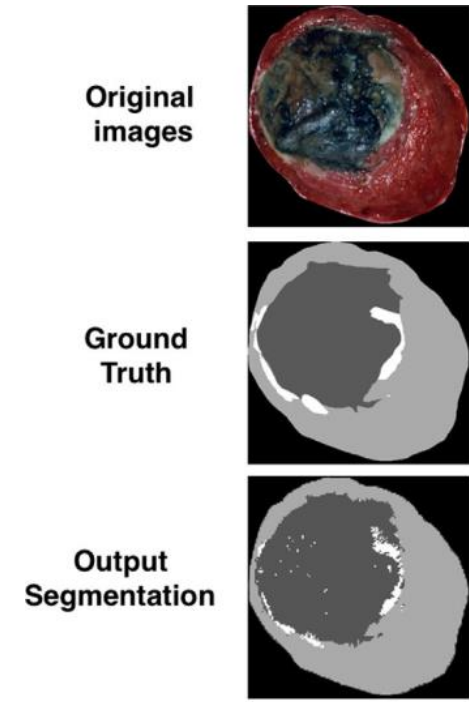

(a)
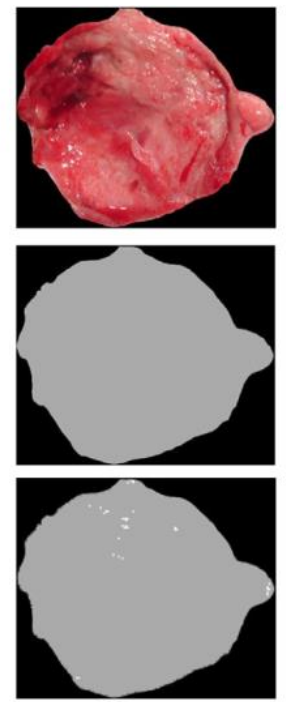

(b)
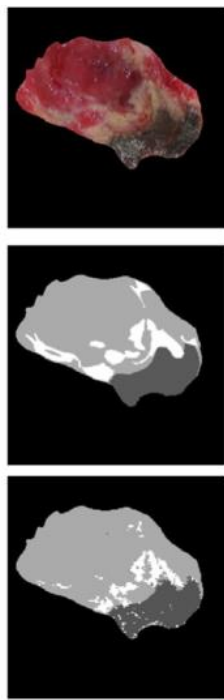

(c)
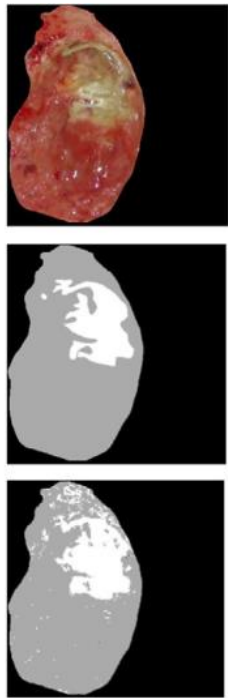

(d)

Figure 26: Examples of original images and their corresponding ground-truth and output segmentation using Deep Learning.

Each of these tables corresponds to columns (a)-(d) of Fig. 9 respectively. We obtained the validation metrics shown in Section 3 for the tissue classes. Note that in Fig. 9 (a) and (c) the pressure injuries contain the three tissue classes, whereas Fig. 9 (b) only contain 
granulation tissue and in Fig. 9 (d) the wound contain granulation and slough tissue classes. In particular, in the Tables 2 and 4 corresponding to Fig. 9 (a) and (c) we provide the results for the segmentation of the three different tissue classes. In Fig. 9 (a) we achieved a DSC of $96.34 \%$ for granulation via the proposed system, $97.49 \%$ for necrosis and 65.03 for slough classes; in Fig. 9 (c) we achieved a DSC of $96.91 \%$ for granulation, $86.15 \%$ for necrosis and 61.67 for slough classes.

Table 2: results of the segmentation of image Fig. 25 (a) containing granulation, slough and necrosis

Predicted tissue types

\begin{tabular}{cccc}
\cline { 2 - 4 } Evaluation metrics & Granulation & Necrosis & Slough \\
\hline DSC & 96.34 & 97.49 & 65.03 \\
Sensitivity & 94.83 & 96.79 & 60.33 \\
Specificity & 97.52 & 95.48 & 98.92 \\
Precision & 97.72 & 94.08 & 72.62 \\
DSCw & 95.49 & & \\
Processing time & $61 \mathrm{~s}$ & & \\
\hline
\end{tabular}

Table 3: Results of the segmentation of image Fig. 9 (b) containing only granulation

\begin{tabular}{cccc}
\hline \multirow{2}{*}{ Evaluation metrics } & \multicolumn{3}{c}{ Predicted tissue types } \\
\cline { 2 - 4 } & Granulation & Necrosis & Slough \\
\hline DSC & 98.35 & N/A & N/A \\
Sensitivity & 98.29 & N/A & N/A \\
Precificion & N/A & 99.99 & N9.74 \\
DSCw & 100 & N/A & \\
Processing time & 98.35 & & \\
\end{tabular}


Table 4: Results of the segmentation of image Fig. 9 (c) containing granulation, slough and necrosis

\section{Predicted tissue types}

\begin{tabular}{cccc}
\cline { 2 - 4 } Evaluation metrics & Granulation & Necrosis & Slough \\
\hline DSC & 96.91 & 86.15 & 61.67 \\
Sensitivity & 98.11 & 94.57 & 58.12 \\
Specificity & 70.99 & 99.48 & 98.05 \\
Precision & 92.65 & 99.11 & 80.01 \\
DSCw & 87.74 & & \\
Processing time & $46 \mathrm{~s}$ & & \\
\hline
\end{tabular}

Table 5: Results of the segmentation of image Fig. $9(d)$ containing only granulation and slough

\begin{tabular}{cccc}
\hline & \multicolumn{3}{c}{ Predicted tissue types } \\
\cline { 2 - 4 } Evaluation metrics & Granulation & Necrosis & Slough \\
\hline DSC & 96.73 & N/A & 81.19 \\
Sensitivity & 81.66 & N/A & 95.49 \\
Specificity & 95.53 & 99.93 & 82.87 \\
Precision & 98.87 & N/A & 81.09 \\
DSCw & 98.94 & & \\
Processing time & $83 \mathrm{~s}$ & & \\
\hline
\end{tabular}

Note that, slough tissue was partly misclassified because there are different textures related to it. Depending on the different types of bacteria, the infection generated by slough tissue has different colors such as off-white, green, light yellow and dark yellow, which can confuse the system. In the Table 3 we show the results when only granulation tissue is 
contained in the wound, and in this case we achieve a DSC of $98.35 \%$ for this kind of tissue. The results obtained when two classes of tissue are contained in the wound are shown in Table 5, and in this case the injury contains granulation and slough, each of them classified by the system with DSCs of $96.73 \%$ and $81.19 \%$ respectively. With this system we obtained an average DSCw of $91.38 \%$, and an average precision per class of $97.31 \%$ for granulation tissue, $96.59 \%$ for necrotic tissue, and $77.90 \%$ for slough tissue. The sensitivity and specificity for all examples were also computed for each tissue type in each case, and the tissue segmentation processing times were also evaluated, with these times ranging from 46 to $83 \mathrm{~s}$ on an Intel®; CoreTM i7-5500 CPU at $2.40 \mathrm{GHz}$ an NVIDIA®; GeForce®; GTX 780 graphics card.

\subsubsection{Discussion}

Despite the fact that our method for pressure injury tissue classification was used on a different database from the ones used in the state of the art techniques, our results provided compelling evidence that the strategy proposed is more reliable when compared to other research reported, with regard to their image segmentation approaches. Skin wound segmentation research was previously carried out using machine learning and other methods and revealed good results. R. Mukherjee et al. (Mukherjee 2014) proposes segmentation performed by converting the RGB images to HSI, then using Fuzzy Divergence Based Thresholding and mathematical morphology operations combined. As for classification, they use color and textural features exacted from fifteen color spaces, and the color-based features were namely: mean, standard deviation, skewness, kurtosis and variance. Conversely, ten textural features were extracted: Shannons entropy, three 
local contrast features and six local binary pattern features. Regarding the classification algorithms, they used Bayesian and Support Vector Machine algorithms to classify the three major part of the wound: granulation, slough and necrosis. The results showed an overall accuracy of $87.61 \%$.

F. J. Veredas et al. (Veredas 2015) chose to combine the mean-shift smoothing procedure and the region-growing algorithm for the segmentation and carried out tissuetype classification using three different machine learning methods namely: Neural Networks, Support Vector Machine and Random-Forest decision trees. The evaluation metrics showed an overall accuracy of $88.08 \%$ using SVM classification method with their database, as compared to $92.01 \%$ using our approach and database.

Thus, our proposed system will enable physicians and medical staff to assess pressure injuries efficiently by providing them with accurate measurements of the different tissue types present in the wounds, and consequently predicting their healing progress and examine the effectiveness of the applied treatment. However, the model has some limitations. Due to depth in some pressure injuries, there are some parts that appear dark in the images. Our system then confuses them with necrotic tissue, which is generally very dark in color. Several classes of infections also exist that are produced by different bacteria, and the slough tissue color changes depending on the kind of the bacteria. These changes affect the classification and should be split depending on the bacteria producing the infection in question. 


\subsection{Chapter summary}

In this chapter, we have presented an approach for automatic tissue segmentation using a Convolutional Neural Network. As the proposed methodology is based on the classification of different tissue types (necrotic, granulation and slough), it enables complicated structures within the image to be recognized. By using this methodology, we were able to obtain very good results and demonstrate that the proposed method is robust in terms of the various aspects that the same tissue type may have, with minimum preprocessing and no post-processing.

The next step which is an essential step in the assessment of pressure injuries is the segmentation and the measurement of the wound. By doing several study visits in collaborating hospitals, we were expanding our dataset in order to be able to have a generalizable and reliable assessment of these wounds. The following chapter will present the final database which was acquired during two years of the $\mathrm{PhD}$ period, and the proposed end-to-end system which combines 2D photographs and 3D meshes and automatically measures the depth, volume, area, major and minor axes. 


\section{CHAPTER 4}

\section{PRESSURE INJURY SEGMENTATION AND}

\section{MEASUREMENT}

\subsection{Introduction}

This chapter presents our proposed approach for the segmentation and the measurement of the quantitative characteristics of the pressure injuries (Zahia 2020). Traditional methods rely on findings retrieved solely from $2 \mathrm{D}$ images. Thus, bypassing the $3 \mathrm{D}$ information deriving from the deep and irregular shape of this type of wounds leads to biased measurements. In this chapter, we propose an end-to-end system which uses a single 2D image and a 3D mesh of the pressure injury, acquired using the Structure Sensor, and outputs all the necessary findings such as: external segmentation of the wound as well as its real-world measurements (depth, area, volume, major axis and minor axis). More specifically, a first block composed of a Mask RCNN model uses the 2D image to output the segmentation of the external boundaries of the wound. Then, a second block matches 
the $2 \mathrm{D}$ and $3 \mathrm{D}$ views to segment the wound in the $3 \mathrm{D}$ mesh using the segmentation output and generates the aforementioned real-world measurements.

The rest of this chapter is organized as follows: a description of the dataset is presented in section 4.2. Section 4.3 overviews the proposed framework by presenting the segmentation part followed by the measurement. Then, section 4.4 discusses the experimental results and analysis.

\subsection{Dataset presentation}

\subsubsection{Dataset collection}

The first step towards achieving this major part of the dissertation was the creation of the dataset. Thanks to collaborating Hospitals in Spain, and during a period of 24 months, almost 40 study visits were made in order to acquire the $2 \mathrm{D}$ photographs and 3D meshes from the patients. Some were also taking from patients homes, for those who had their care-givers assisting them at home. The 2D photographs which were captured during the hospital visits were acquired using a cell-phone camera (Oneplus 5T/ iPhone 6), with a resolution ranging from $1080 \times 2280$ to $2747 \times 3079$. No flash light was used, and the patients were moved in such way that the sunlight would reach the wound. The 3D meshes were acquired using the Structure Sensor (Structure Sensor), which will be presented in the next subsection. The collaborating hospitals were: Cruces Hospital, Santa Marina Hospital and Basurto Hospital in Spain. All subjects gave their informed consent for inclusion before they participated in the study. The study was conducted in accordance with the Declaration of Helsinki, and the protocol was approved by the Ethics Committee of ETK-18/16-17. 


\subsubsection{Structure Sensor}

The structure sensor is a first 3D scanner used on mobile devices. s. While it is performing the best with iOS devices, it can still be used with other devices and platforms, including Android devices, Windows, macOS and laptop/desktop computers. In our proposed research, we mounted it on an iPad Air 2, using its customized bracket. This Structured Light System (SLS) contains a laser-emitting diode, an infrared radiation range projector, and an infrared sensor. Then, using a safe infra-red light, the sensor scans the objects and the iPad's RGB camera sends data to a System On a Chip (SOC) for processing. The Structure sensor is a software controlled scanner and can be mounted on an iPad with its customized bracket and works on a rechargeable battery, as shown in Fig. 26.
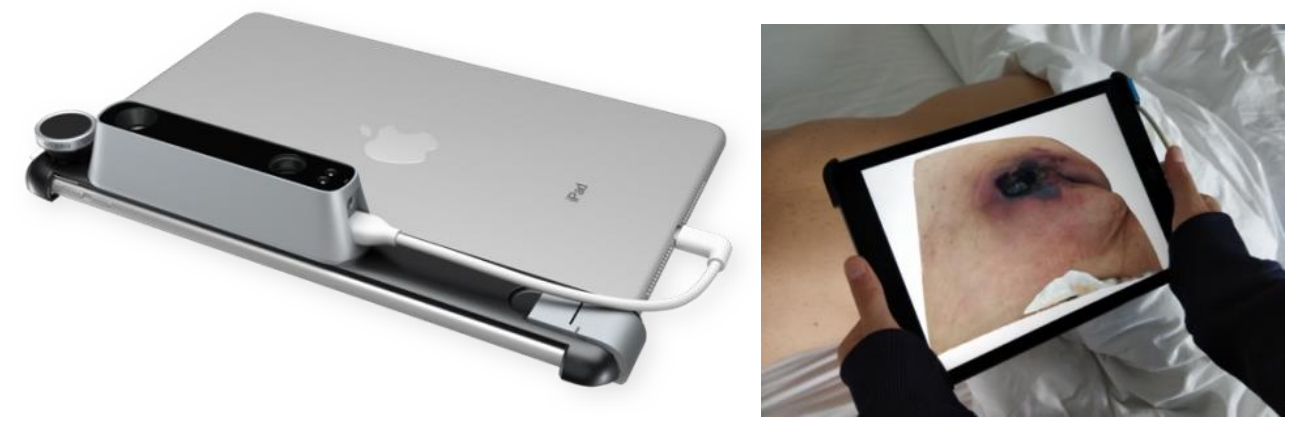

Figure 27: Left:The Structure Sensor mounted on an iPad. Right: Scanning of a pressure injury using the Structure Sensor

The sensor alone delivers a point dataset, of a 640 x 480 pixels resolution, where each pixel contains the distance from the sensor to the target. The role of the infrared (IR) sensor is to record the During the acquisition of the the 3D scene, the target point, shown as a black point in Fig. 27, is projected at depth $\mathrm{Z}$ from the camera plane. 


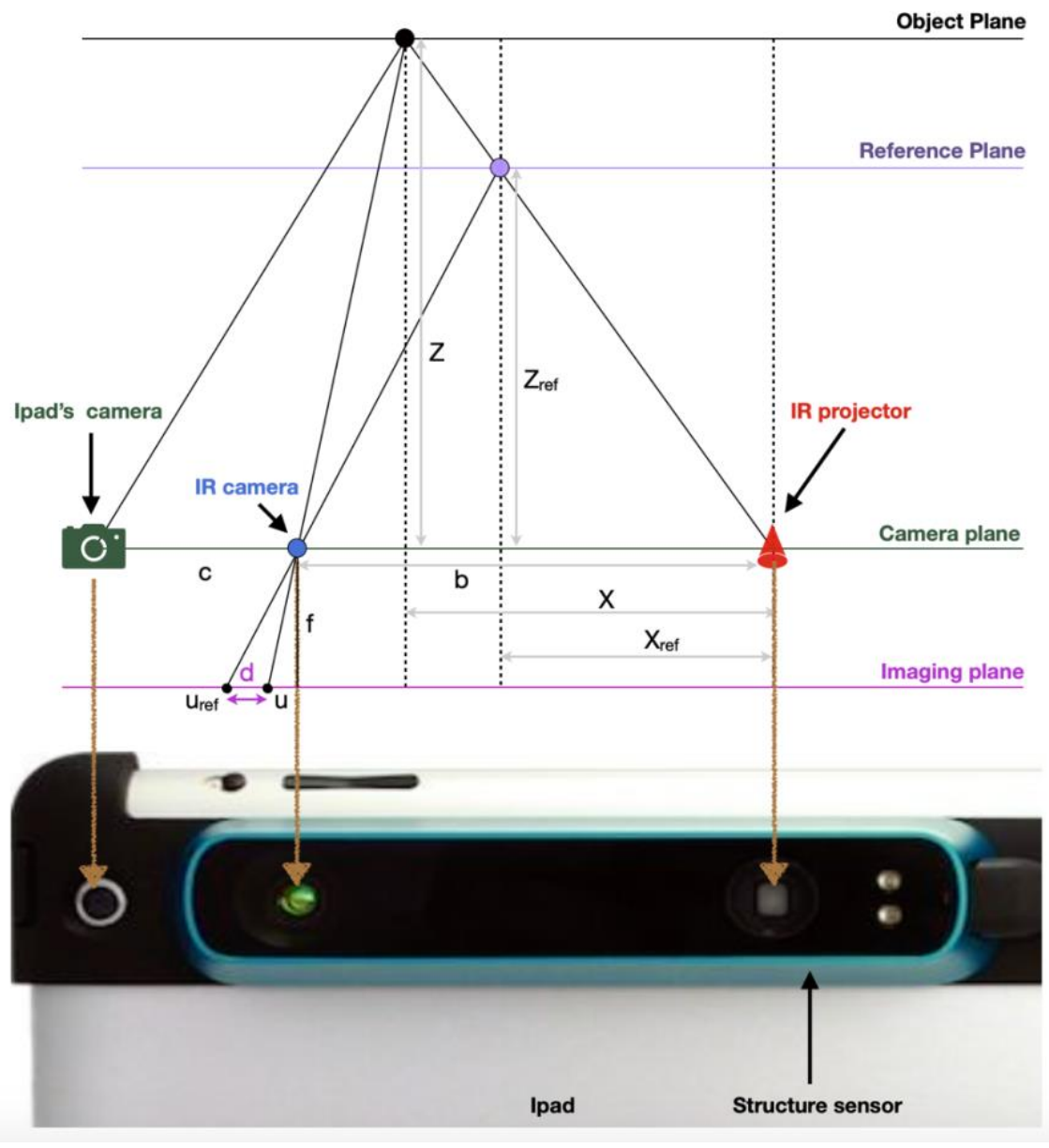

Figure 28: Explanation of depth measurement using the Structure Sensor

The IR camera is situated at a distance $b=65 \mathrm{~mm}$ from the IR projector. Whereas the iPad's RGB camera is situated at a distance $\mathrm{c}=6.5 \mathrm{~mm}$ from the IR camera. Depth images are constructed on the imaging plane through the perspective IR camera. Let's assume that an object is on the reference plane is at a distance Zref to the sensor. If the object is shifted closer or further from the sensor, a displacement in pixels between the two patterns is created on the imaging plane, called the disparity (eq 10):

$$
\mathrm{d}=\boldsymbol{u}-\boldsymbol{u}_{\boldsymbol{r e f}}
$$


Using the trigonometry relations using the triangles in Fig. 27, and by determining the constant

parameters Zref and the focal length $\mathrm{f}$, using calibration, the depth $\mathrm{Z}$ can be obtained using the following equations (eq 11,12,13):

$$
\begin{aligned}
\frac{\mathrm{X}-X_{\text {ref }}}{\mathbf{b}} & =\frac{\mathrm{Z}-Z_{\text {ref }}}{\mathrm{Z}} \\
\frac{\mathbf{d}}{\mathrm{f}} & =\frac{\mathrm{X}-X_{\text {ref }}}{Z_{\text {ref }}} \\
Z & =\frac{Z_{r e f}}{1+\frac{Z_{r e f} \times d}{\mathbf{f} \times \mathbf{b}}}
\end{aligned}
$$

The accuracy of the Structure Sensor has been studied and revealed the reliability of its usage for object 3D reconstruction. On one hand, Kalantari, M. et al. (Kalantari 2016) present the accuracy of measurements from the Structure Sensor data, especially point clouds in the context of volunteered 3D indoor information, compared to the ground-truth. They achieved an error of $2.1 \mathrm{~cm}$ for the smallest room captured, which was from a distance of $2 \mathrm{~m}$ at most. The authors show that the Structure Sensor is accurate for smaller rooms, and when the acquisition is made in a circular way. During the acquisition of the pressure injuries' 3D meshes, we captured the wounds at a distance of $30 \mathrm{~cm}$, by moving the sensor also around the area. This shows that the 3D reconstruction using the Structure Sensor is highly reliable. On the other hand, in (Shah 2013), the authors showed that the 3D scanner gives accurate volumetric measurements in comparison to standard volumetric measurements obtained by the waterfill technique. In addition, the 3D scanner was found to be more reliable and valid compared to other three techniques: the ruler method, acetate grid tracing and 2D planimetric measurements. 


\subsubsection{Dataset Summary}

Finally, the database was composed of 210 photographs of pressure injuries. 110 images were acquired from hospitals, and 100 images from Medetec Medical Images online database (MIOD) (Medetec). We decided to add more images from Online sources in order to improve the model's performance and generalizability. For the segmentation pipeline, 175 images were used for the training and 35 images were used for testing (the 35 images for testing contain 21 pressure injuries, as some PIs have several images). Those 21 pressure injuries were scanned using the Structure Sensor. From those meshes, 6 meshes were not valid for the measurement of the depth and volume as they were either on the heel or on the hip, where the femur bone was pushing the inside of the ulcer above its surface, resulting in 15 meshes used for the 3D measurement, as shown in figure 28.

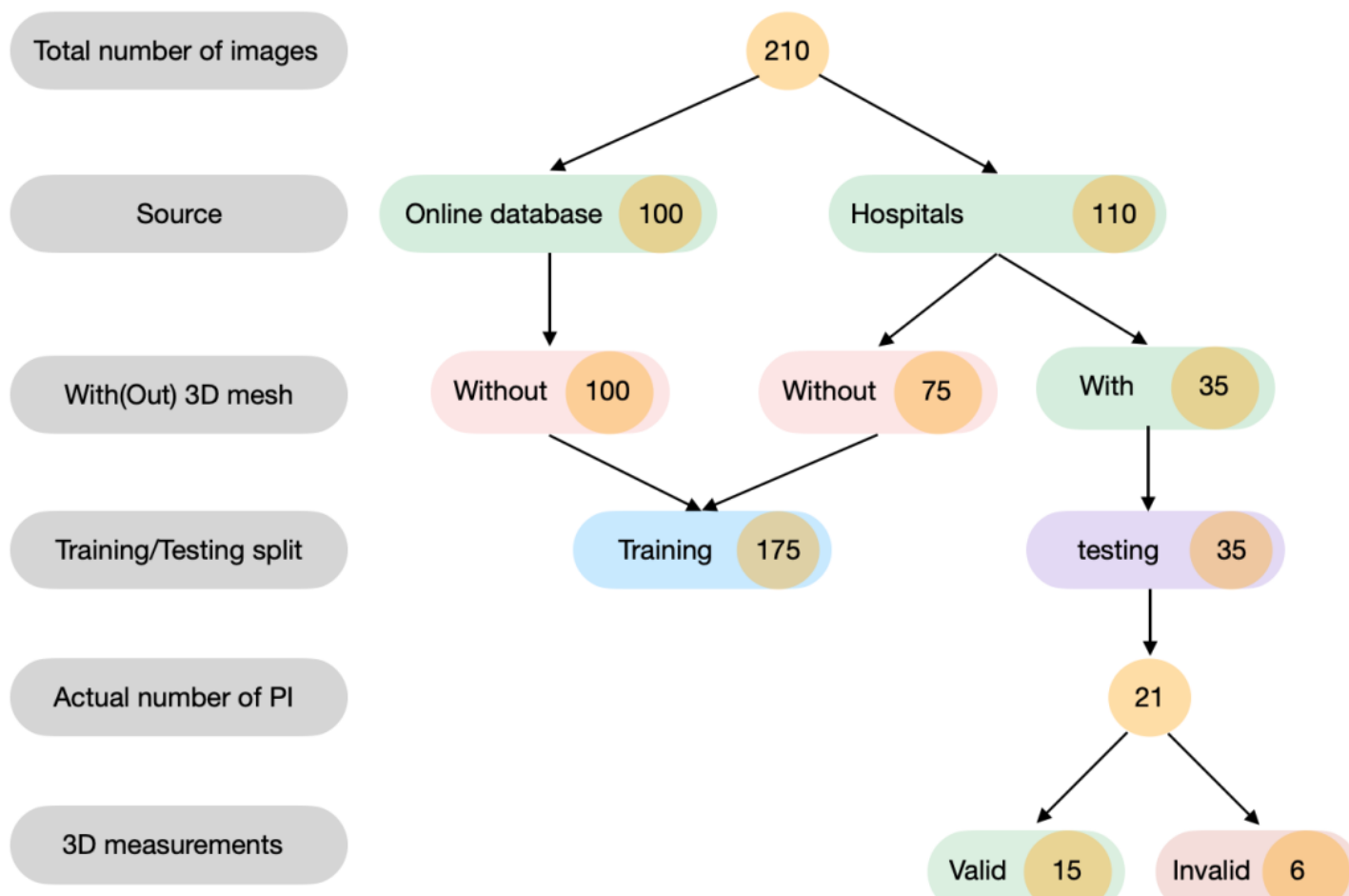

Figure 29: An illustrative diagram of the composition of the database used to conduct this research. 


\subsection{The Proposed Framework}

\subsubsection{Global Framework}

We propose an end-to-end framework which automatically retrieves quantitative information of the pressure injury using solely a 2D image and a $3 \mathrm{D}$ mesh of the wound, as shown in Figure 29. The developed system uses CNNs in order to automatically detect and segment the wound. Then it combines the segmentation results with the $3 \mathrm{D}$ mesh and automatically computes the wound depth, area, volumes and major and minor axes. Firstly, the 2D image is fed to a Mask-RCNN model in order to segment the pressure injury. Simultaneously, the 3D mesh is rasterized and a top view image and the matrix of face indices corresponding the top view are generated. After matching the top view image with the $2 \mathrm{D}$ image of the wound, captured using a camera, a projective transform matrix is calculated. Using the segmentation result, the top view image is segmented too, and the faces belonging to the inside and the boundaries of the wound are detected. The measurement block finally computes the depth, area, volume and axes of the wound.

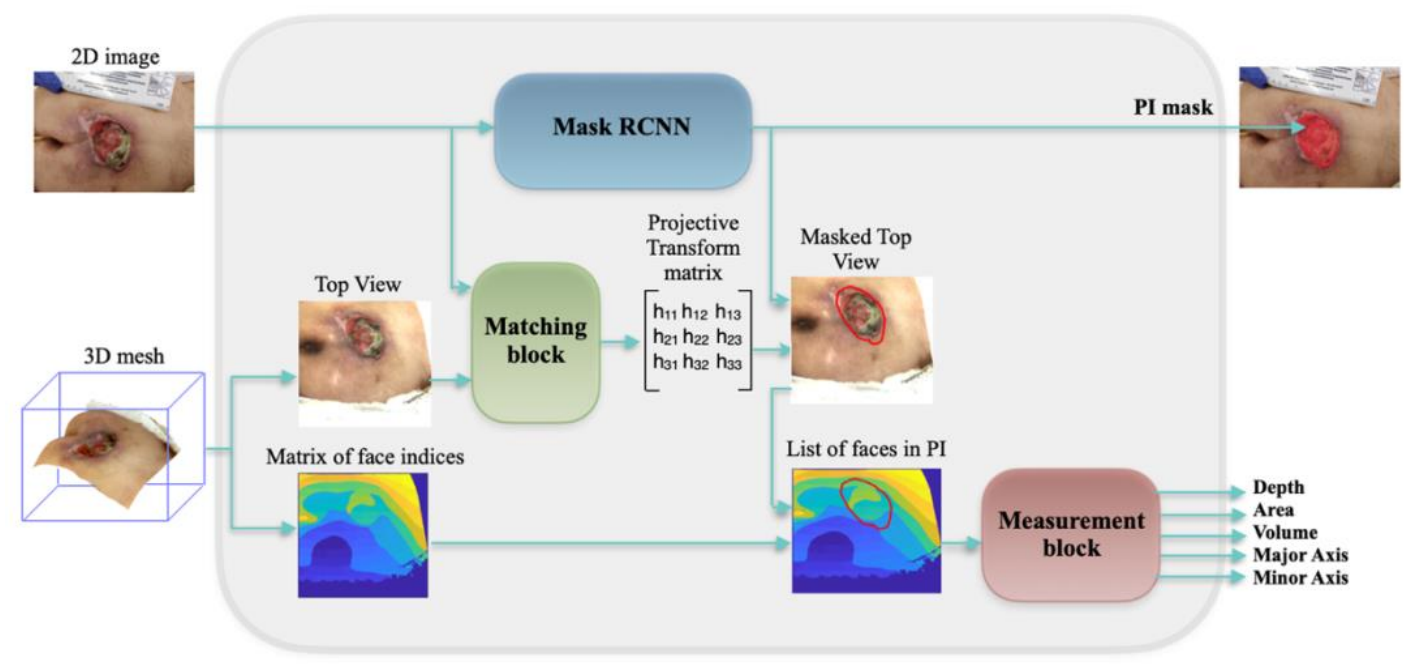

Figure 30: Schematic view of our proposed framework 


\subsubsection{Pressure injury segmentation}

Traditionally, wound image segmentation was performed using image processing techniques (Zahia 2019). Several image processing approaches have been proposed in the literature, such as Region-based segmentation (Gholami 2017)(Wang 2016)(Khan 2015)(Fauzi 2015), and edge-based segmentation (Gholami 2017)(Nandagopan 2016)(Lee 2015). In addition, traditional machine learning methods were used to perform wound segmentation. Both unsupervised methods such as clustering (Haider 2016)(Yadav 2013), and supervised methods such as Support Vector Machines (SVM) (Bochko 2010)(Seixas 2015) and Bayesian classifiers (Veredas 2015) were used to segment the wound after extracting the features such as mean-color information, color histogram statistics and Scale Invariant Feature Transform (SIFT) features (Lowe 2004). The issue with traditional approaches is the necessity to choose the features which are important in the given images. Hence, the computer vision engineers need to judge with a trial and error process to decide the features which best describe the objects to detect.

Deep Learning has introduced the concept of end-to-end learning. When the machine is given a set of images, which have been annotated by experts, Neural networks discover the underlying patterns during the training and automatically find out the most descriptive features with respect to the objects to be detected. These methods have achieved state-of-the-art performance in a variety of medical image processing tasks, including medical image segmentation.

In order to tackle the segmentation task, we used the Mask RCNN algorithm (He 2017) to detect and segment pressure injuries. It has very important research value and has broad application scenarios in the field of medical imaging. Mask RCNN is an instance 
segmentation framework which is an extension of Faster RCNN (Ren 2017). It is divided into two stages: the first stage is a small neural network called a Region Proposal Network (RPN) which scans the image and generates the proposals: the regions that most likely contain the wound. In the second stage, features are extracted from each proposed region in order to perform in parallel: proposal classification, bounding box regression and a binary pixel-level mask generation. The network structure block diagram of the Mask RCNN algorithm is shown in Figure 30.

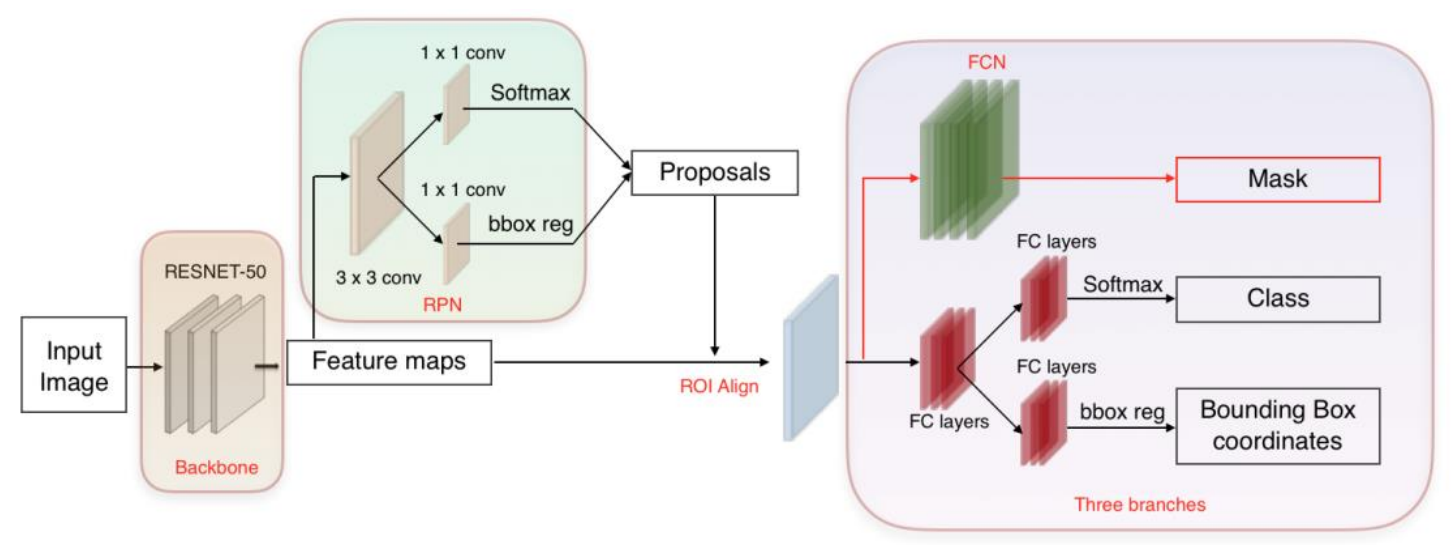

Figure 31: Overview of Mask RCNN architecture chosen to segment the pressure injury: Proposals about the regions which possibly contain the wound based on the input image are detected. Then three branches predict the class of the object, the bounding box and the mask in pixels of the wound. Both stages are connected to the backbone structure.

\subsubsection{Measurement framework}

The proposed framework for pressure injury measurement starts with mesh rasterization, where we retrieve the top view image of the wound from the $3 \mathrm{D}$ mesh, and its corresponding matrix of face vertices. This image will then be matched to the $2 \mathrm{D}$ photograph which we previously used in the segmentation pipeline and got its mask prediction from the Mask RCNN. Once the two images are matched, the pixels corresponding to the edges of the wound are retrieved using the transform matrix that we 
have calculated in the previous step. Now that we have the vertices corresponding to the wound, we follow a series of operations in order to calculate each one of the desired characteristics: depth, volume, area, major and minor axes.

\subsubsection{Mesh Rasterization}

When rendering the $3 \mathrm{D}$ mesh, we retrieve the top view image, where the surface of the pressure injury lies perpendicular to the camera direction, as shown in Figure 31. This step requires multiple transformations from world space to camera space, then to screen space. Firstly, the objects have point coordinates which are defined in world space, with respect to a global or world Cartesian coordinate system. The camera is no different than any other 3D object. By considering the camera as a reference system, points are transformed from the world coordinate system to the camera coordinate system, by multiplying the point world coordinates by the inverse of the local-to-world matrix. Then, to transform vertex coordinates from camera to screen-space, a so-called projection matrix is defined, which transforms coordinates to normalized device coordinates. All the aforementioned transformations are integrated within Pytorch3D library (Pytorch3D). Then, we created a Phong renderer by composing a rasterizer and a shader. The textured Phong shader (Bishop 1986) interpolates the texture coordinates for each vertex from the texture image and applies the Phong lighting model. 


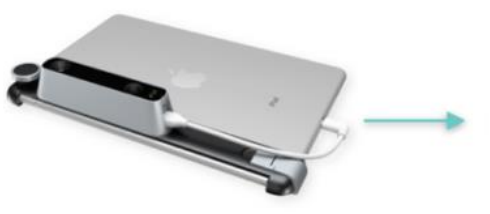

Obj file

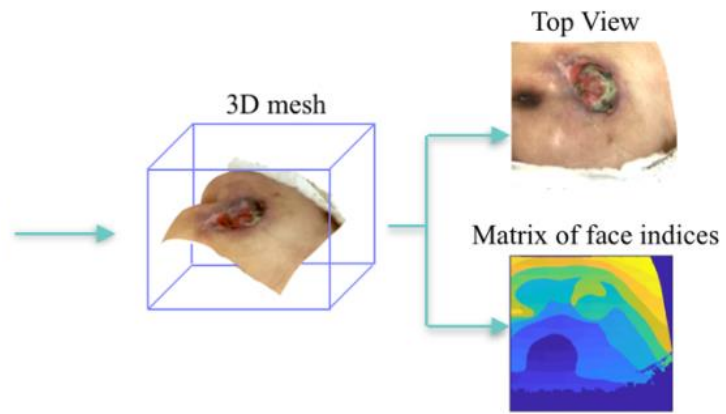

Figure 32:. Mesh rasterization process: the Obj file of the 3D mesh acquired with the Structure Sensor is rendered in order to retrieve the top view image and the matrix of face indices.

Since the 3D meshes of the pressure injuries were acquired under the same conditions (near plane of the camera was always parallel to the surface of the wound, and the distance from the wound ranged between 30 to $40 \mathrm{~cm}$ ), the selected camera viewpoint to retrieve the top view image was set to: Azimuth $=0$, Elevation $=0$ and Distance $=0.3$ $\mathrm{m}$. The corresponding tensor with the list of face indices which overlap with the pixels of the top view, was also generated. The shape of this tensor is (image size, image size, faces per pixel), where faces per pixel corresponds to the faces of the mesh which were projected into the same pixel in the output top view image. In our application, we chose faces per pixel $=5$ in order to extract all the faces inside the mesh which are not seen in the top view image. This part of the framework was implemented using PyTorch3D library, an opensource toolkit for 3D based Deep Learning research (Pytorch3D).

\subsubsection{Matching Block}

Once the top view of the wound was generated, the next step was to match it with the 2D photograph of the wound. We used Progressive Sparse Spatial Consensus (PSSC) (Ma 2017) as it has proven to be efficient in case the two images contain a large 
number of outliers. In our application, the 2D image may contain several objects which are not present during the acquisition of the 3D mesh, such as: ruler, doctor's hand, background, etc. In PSSC, the maximum likelihood spatial consensus estimation is done to formulate the matching, and the Expectation-Maximization (EM) approach is adopted for optimization. Once the matching pixels from both images were retrieved, a projective transform matrix was calculated in order to transform the pressure injury mask from the 2D image to the top view. Using the pairs of matching landmarks in both images, the parameters of the matrix were calculated using the MATLAB function fitgeotrans, by specifying the desired transformation, which is projective in this case. This way, we could segment the pressure injury on the top view image by extracting the faces belonging to the boundaries and the inside of the wound, and proceed with the measurement block, as shown in Figure 32.

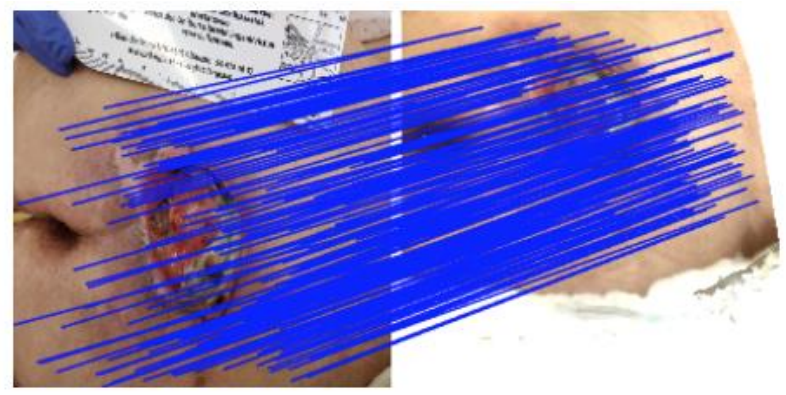

(a)

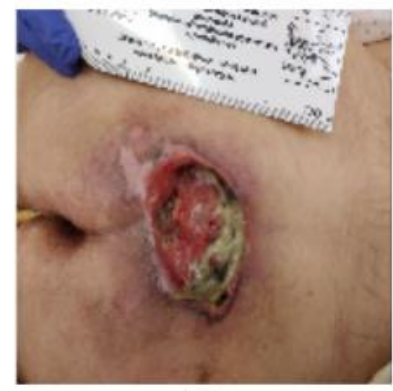

(b)

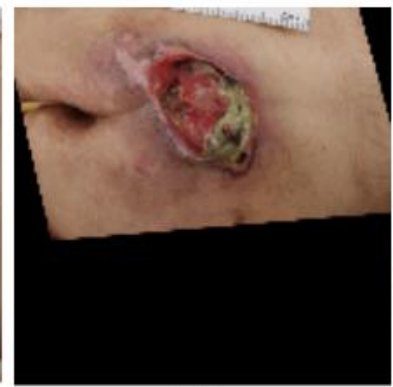

(c)

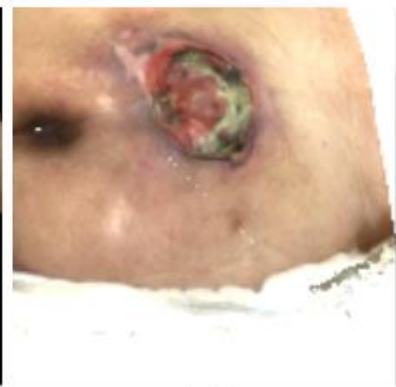

(d) 


\subsubsection{Measurement Block}

Using the matrix of face indices retrieved during the mesh rasterization, both the indices corresponding to the faces in the boundary and in the inside of the wound were extracted. For an easier processing of the mesh, we decided to rotate it in such way that the surface of the pressure injury is parallel to the $\mathrm{XY}$ axis, as shown in Figure 33.

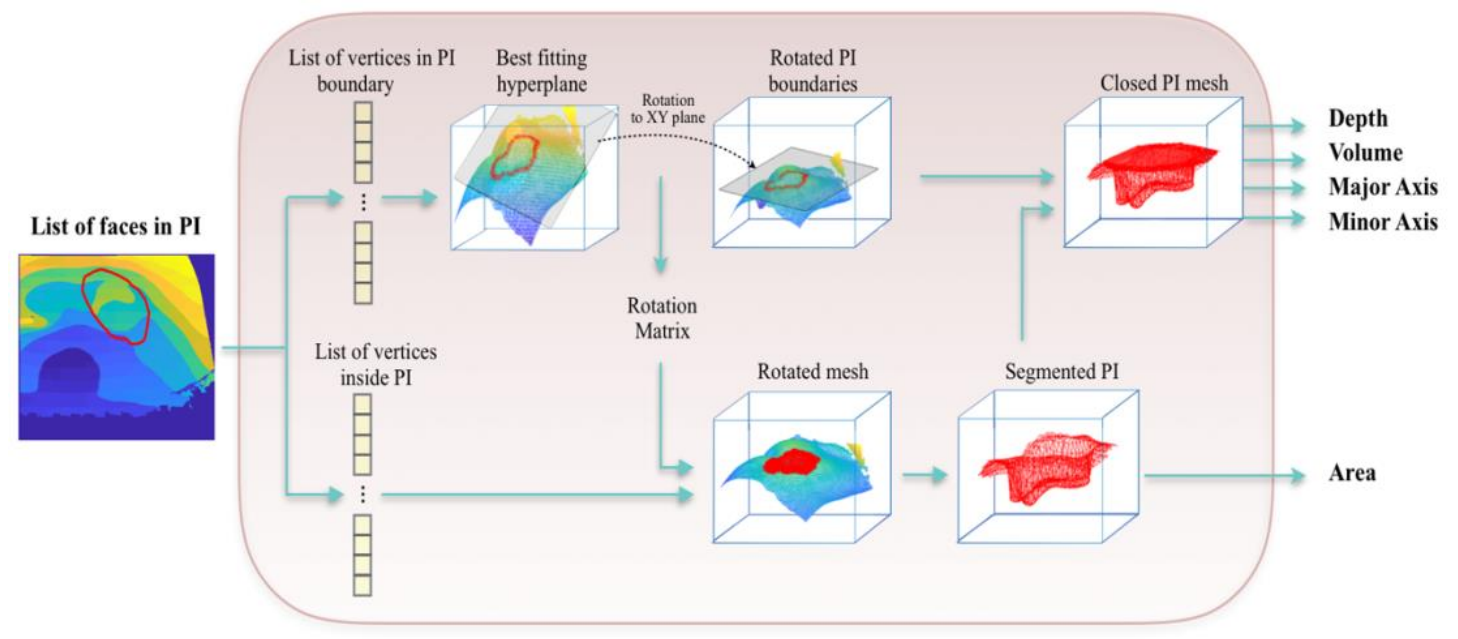

Figure 34: Schematic view of the measurement block: after extracting the vertices belonging to the boundaries and the inside of the wound, the 3D mesh is rotated and the best fitting hyperplane is calculated to close the surface of the pressure injury.

The best fitting hyperplane is calculated using RANSAC algorithm (Fischler 1981). This algorithm tries to find the model that fits the maximum number of data points. Rather than using all the data once to obtain the solution and then eliminating the invalid data points, the RANSAC algorithm uses a small initial number of data points, estimates the model which fits the maximum number of inliers, then enlarges this set with consistent data and redo the estimations again until reaching the solution. Using the points in the 3D space which belong to the boundaries of the pressure injury, the RANSAC algorithm finds the maximum number of inliers which belong to the same hyperplane, which will be then the surface closing the top of the wound. Hence, the depth of each vertex 
inside the wound would be equal to its $\mathrm{z}$-coordinate value - the $\mathrm{z}$ value of the hyperplane containing the boundary of the ulcer. The maximum absolute value of the resulting depths would represent the depth of the wound. The area of the wound is calculated by the summation of the areas of all the triangles inside the wound. In order to measure the volume, the mesh has to be closed. To achieve this, we created a new surface by computing the convex hull (Avis 1997) on the xy coordinates of the vertices in the boundary of the wound. The height and width of the minimum bounding box enclosing this surface would represent minor and major axes. Then, the volume is measured by summing up the volumes of the tetrahedrons that go from the origin vertex, which was set to be the center of the closed top surface, to the triangles inside the mesh.

\subsubsection{Implementation}

We implemented the segmentation pipeline using Python 3.6 with Keras 2.0 (Chollet 2015) and TensorFlow 1.3 (Abadi 2016). The backbones of Mask RCNN that were tried were ResNet50 and ResNet101. In addition, we tested the wound detection and segmentation capabilities of the model when trained on MS COCO and ImageNet datasets. The best performing backbone was ResNet-50, trained on MS COCO dataset. The best performing backbone was ResNet-50 and the number of epochs was set to 35. The 3D mesh processing and rasterization were done using PyTorch3D library and both the matching and measurement blocks were implemented using Matlab 2018b. We used a standard PC with dual Nvidia Geforce GTX 1080 Ti graphical processing unit (GPU) support. 


\subsection{Results and Discussion}

\subsubsection{Evaluation metrics}

The segmentation method was evaluated using the following performance measures: Dice Similarity Coefficient (DSC), sensitivity and precision (Zahia 2018). As for the measurement evaluation, we evaluated our results using the Mean Absolute Error (MAE) and Root Mean Squared Error (RMSE). The ground-truth measurements of the pressure injuries were made manually using Blender software. The mesh segmentation corresponded to the ground-truth segmentation of the 2D images, which was validated by two physicians who were told to independently examine the ground-truth images and document their professional criteria regarding our manual segmentation. The ground-truth measurements were made using BLENDER software, as depicted in fig. 35 .

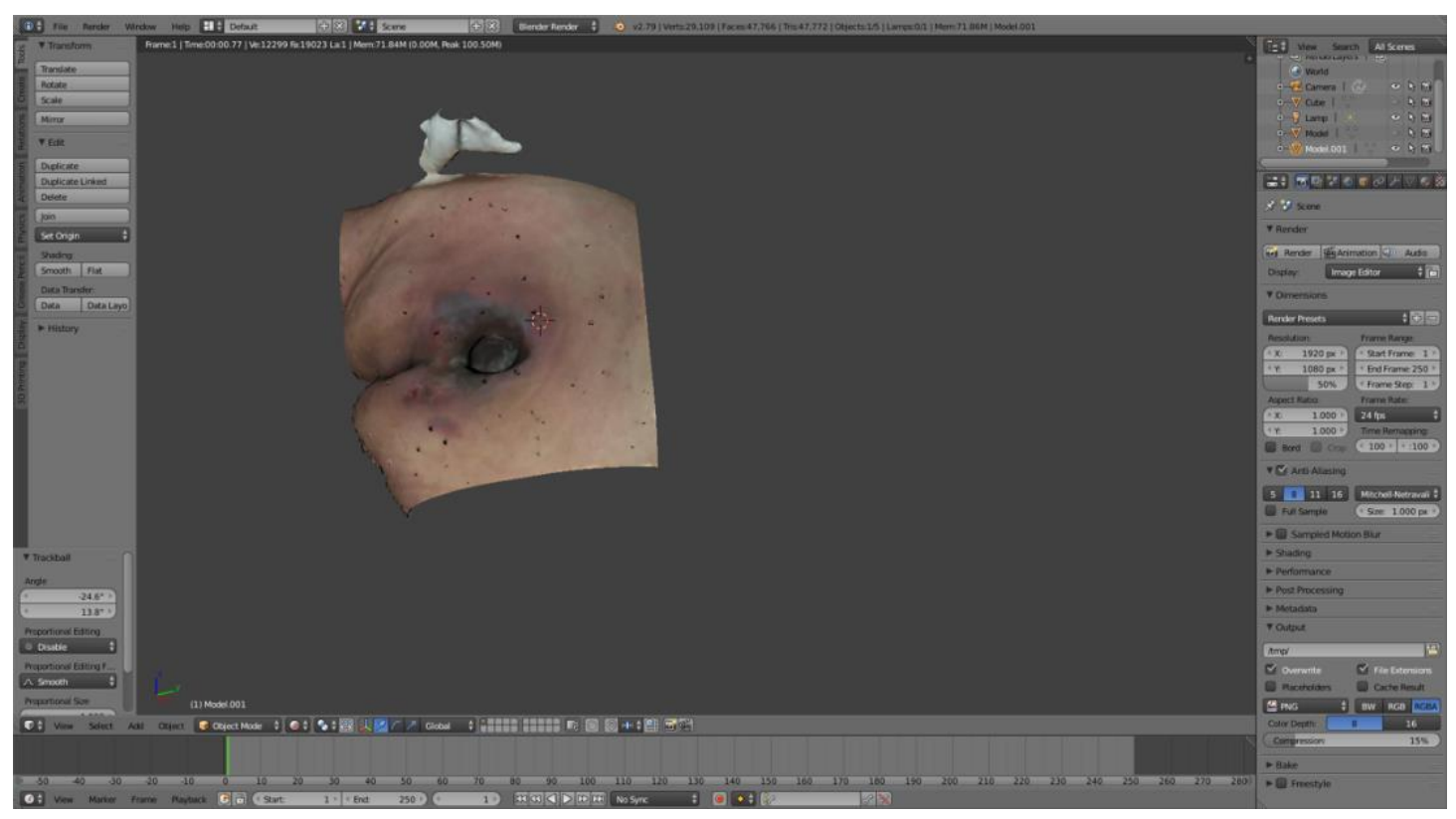

Figure 35: View of the mesh using BLENDER software 
First, faces, vertices and edges were selected to crop the pressure injury, as shown in fig. 36. In this figure, we illustrate the cropping process using three different pressure injuries. Then, using the Ruler tool the minor and major axes were measured.
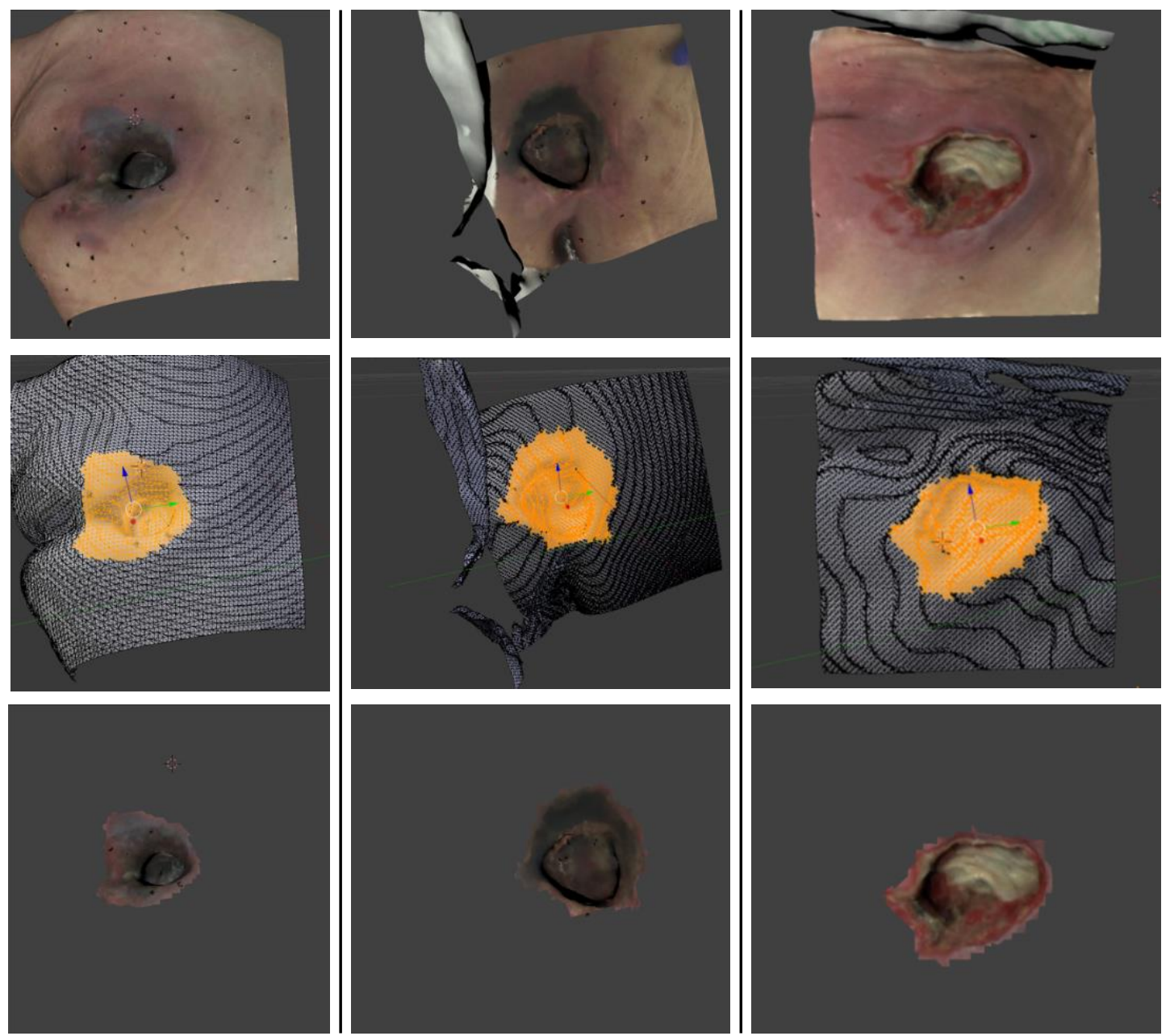

Figure 36: Examples of 3D mesh cropping of pressure injuries (Left: wound (a), Middle: wound (b), Right: wound $(c))$

Next, from the 3D printing tab, the Area tool from Statistics was used to measure the area of the wound. Subsequently, in order to close the mesh, the Remesh tool was used, and the number of octrees was set to 6 in order to maintain the exact shape of the wound with a smooth closed surface, as shown in fig. 37.Similarly to area and axes measurement, 
the values of the volume and depth were retrieved using Volume tool and Ruler tool, respectively.
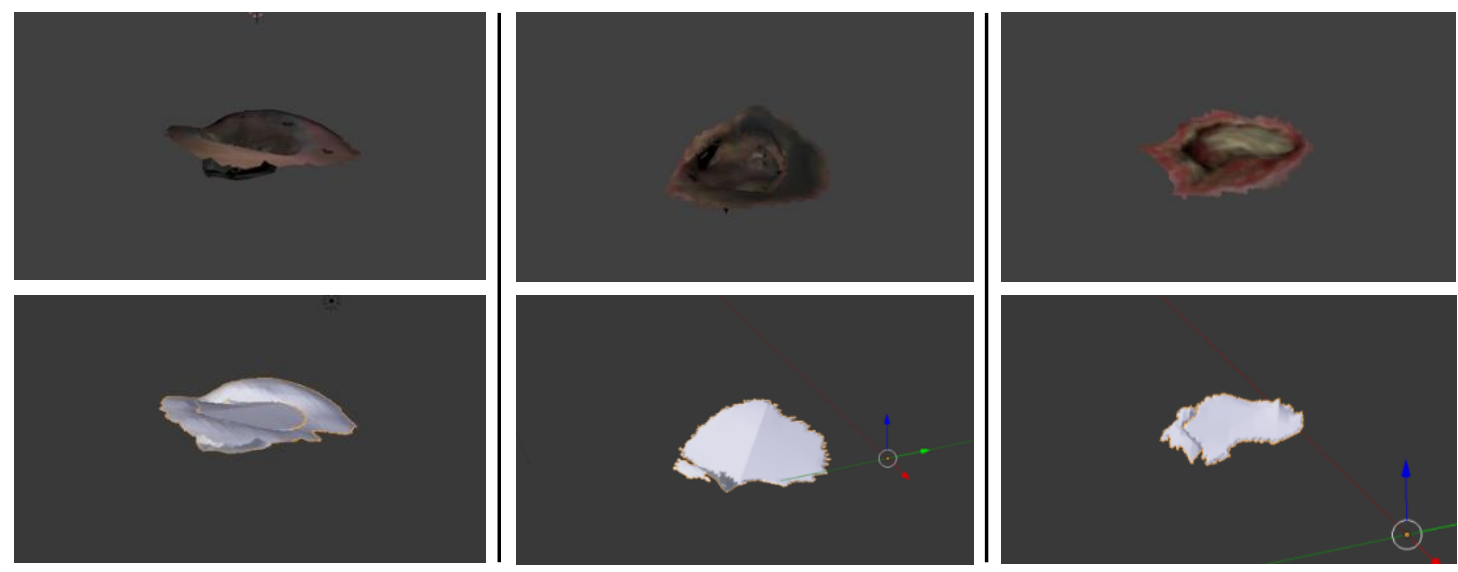

Figure 37: 3D mesh closing for volume measurement (Left: wound (a), Middle: wound (b), Right: wound (c))

\subsubsection{Results}

The best results of segmentation were achieved using Mask-RCNN model with ResNet-50 backbone, with a mean Dice score of 0.83 for wound segmentation, a mean sensitivity score of 0.85 and a mean precision score of 0.87 . Figure 34 depicts some segmentation results.

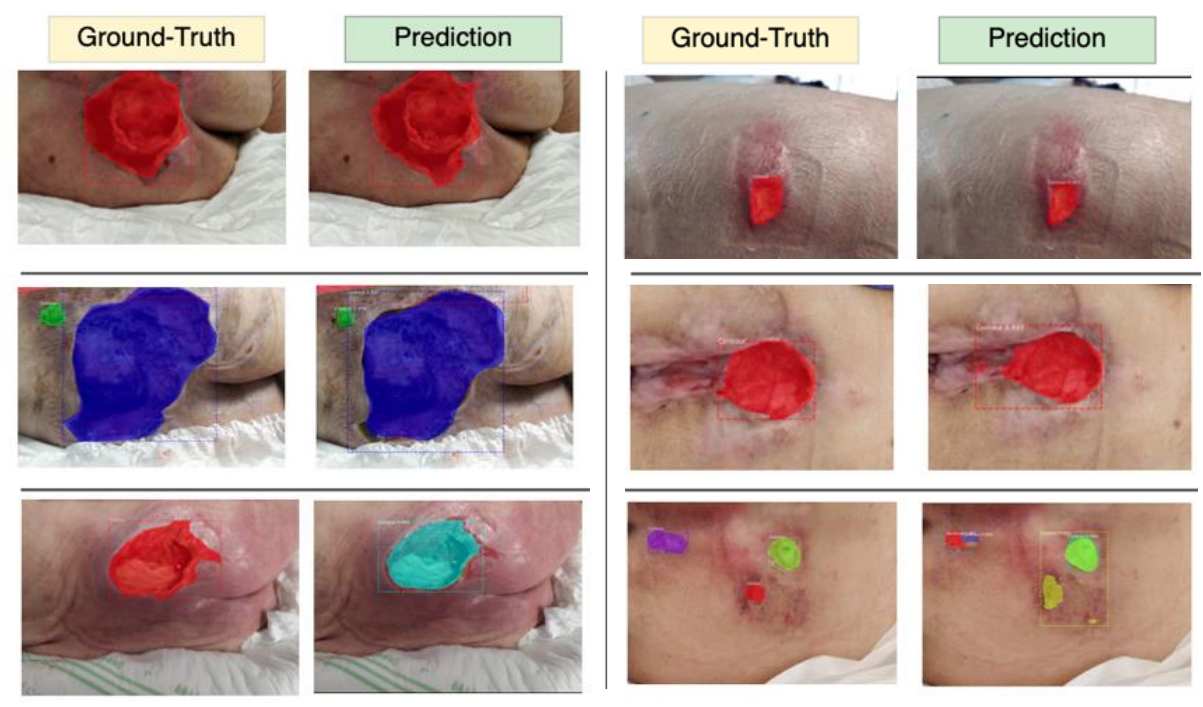

Figure 38: Results of the segmentation of the pressure injuries (Colors were generated randomly) 
As for the measurements part, the MAE values were $0.74 \mathrm{~cm}, 4.69 \mathrm{~cm}^{3}, 5.41 \mathrm{~cm}^{2}$, $1.03 \mathrm{~cm}$ and $1.10 \mathrm{~cm}$ for the depth, volume, area, major axis and minor axis respectively. Whereas the RMSE values were $0.58 \mathrm{~cm}, 1.57 \mathrm{~cm}^{3}, 1.86 \mathrm{~cm}^{2}, 0.82 \mathrm{~cm}$ and $0.87 \mathrm{~cm}$ for the depth, volume, area, major axis and minor axis, respectively. Figures 35-38 show four bar plots corresponding to the measurements of the depth, volume, area, major axis and minor axis, respectively, on the 15 pressure injuries used for validation. Since the pressure injuries had different sizes and shapes, the plots were chosen to have a logarithmic scale, in order to have a better view of those with a value less than $1\left(\mathrm{~cm} / \mathrm{cm}^{2} / \mathrm{cm}^{3}\right)$. The pressure injury IDs are consistent in all the plots, where we chose to order them by their real areas.

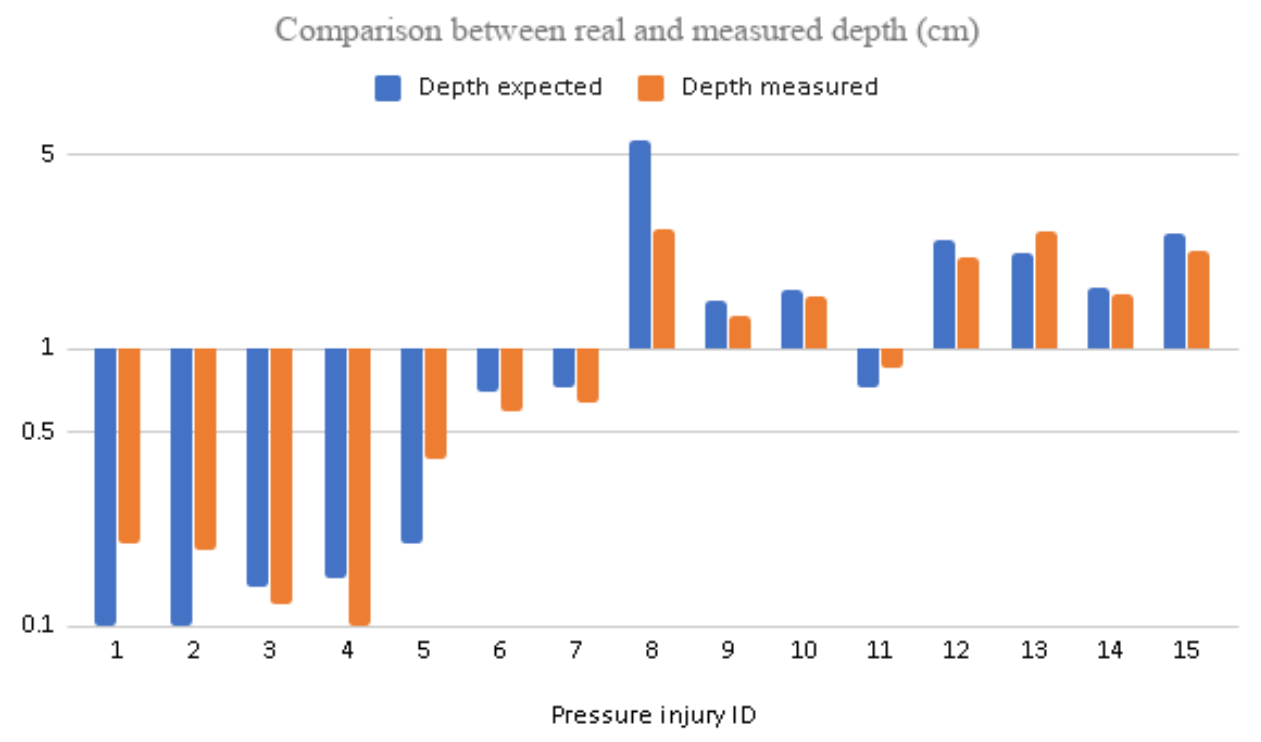

Figure 39:Measurement results of 15 different pressure injuries: depth in logarithmic scale (cm). 
Comparison between real and measured Area $\left(\mathrm{cm}^{2}\right)$

Area expected Area measured

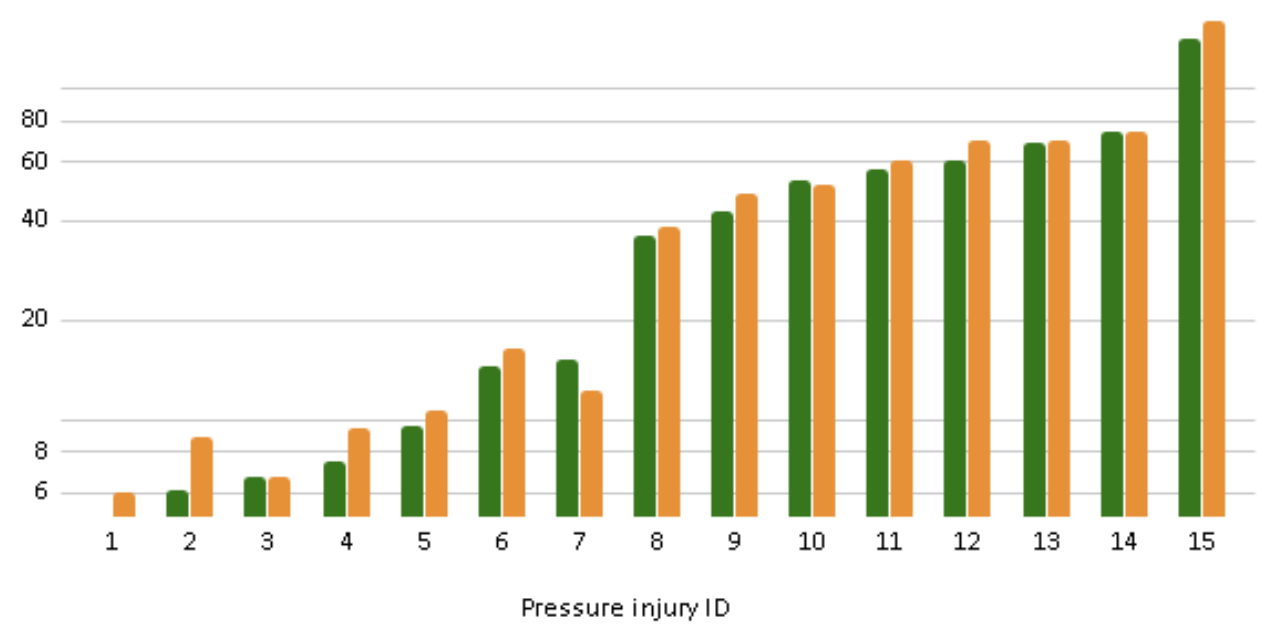

Figure 40: Measurement results of 15 different pressure injuries: area in logarithmic scale $\left(\mathrm{cm}^{2}\right)$

Comparison between real and measured volume $\left(\mathrm{cm}^{3}\right)$

Volume expected Volume measured

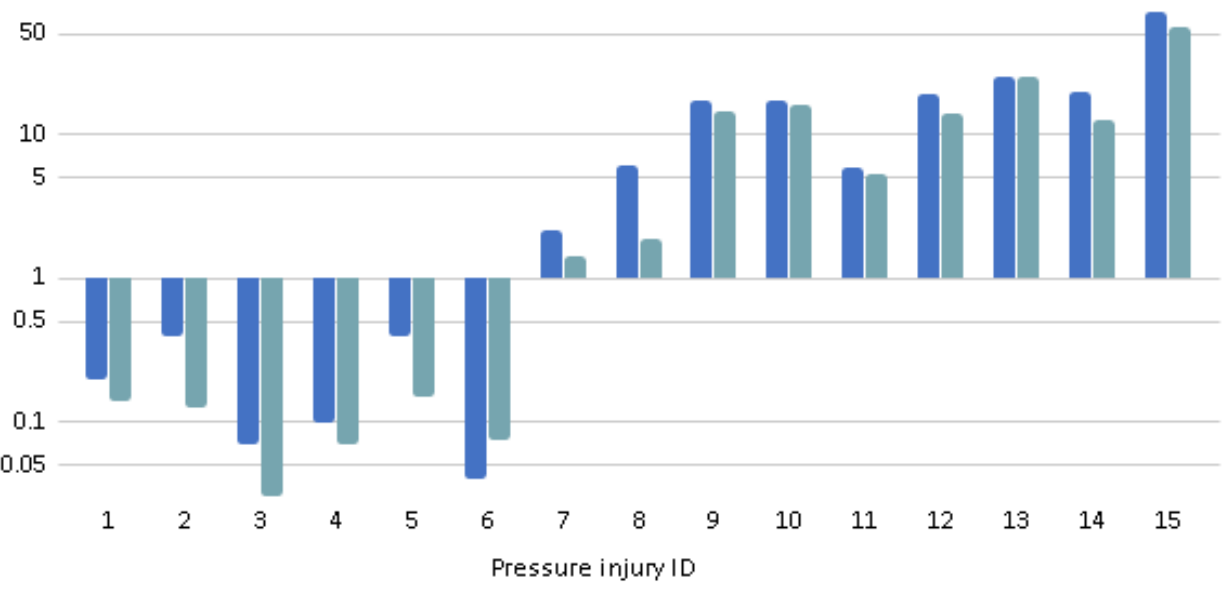

Figure 41: . Measurement results of 15 different pressure injuries: volume in logarithmic scale $\left(\mathrm{cm}^{3}\right)$ 


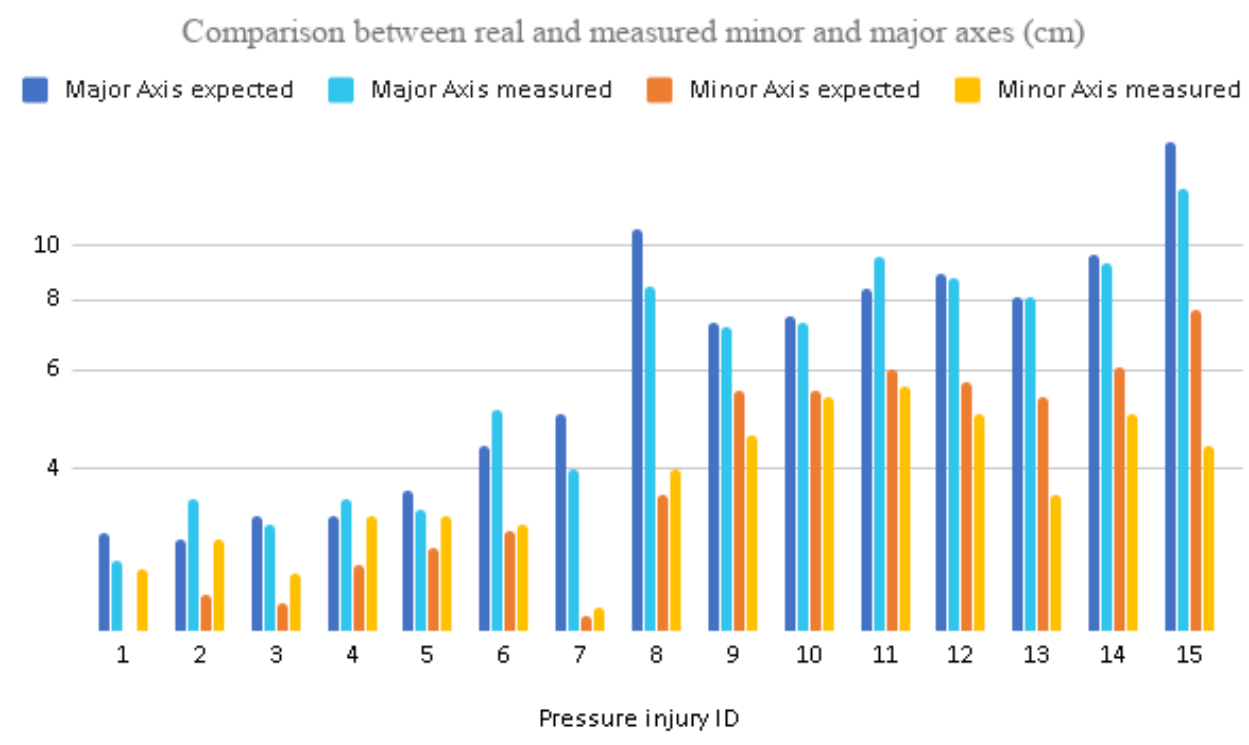

Figure 42: Measurement results of 15 different pressure injuries: minor and major axes in logarithmic scale $(\mathrm{cm})$

Figure 39 shows the box plots of the five components measured. Each subplot contains the distribution patterns of both the expected and measured components. We can notice from all the plots that the distribution of the measured values are similar to the expected ones. However, for both major and minor axes, maximum non-outlier values are shifted by almost $2 \mathrm{~cm}$, which is due to some irregular borders of some pressure injuries that tend to cause also uncertainty in the segmentation task.

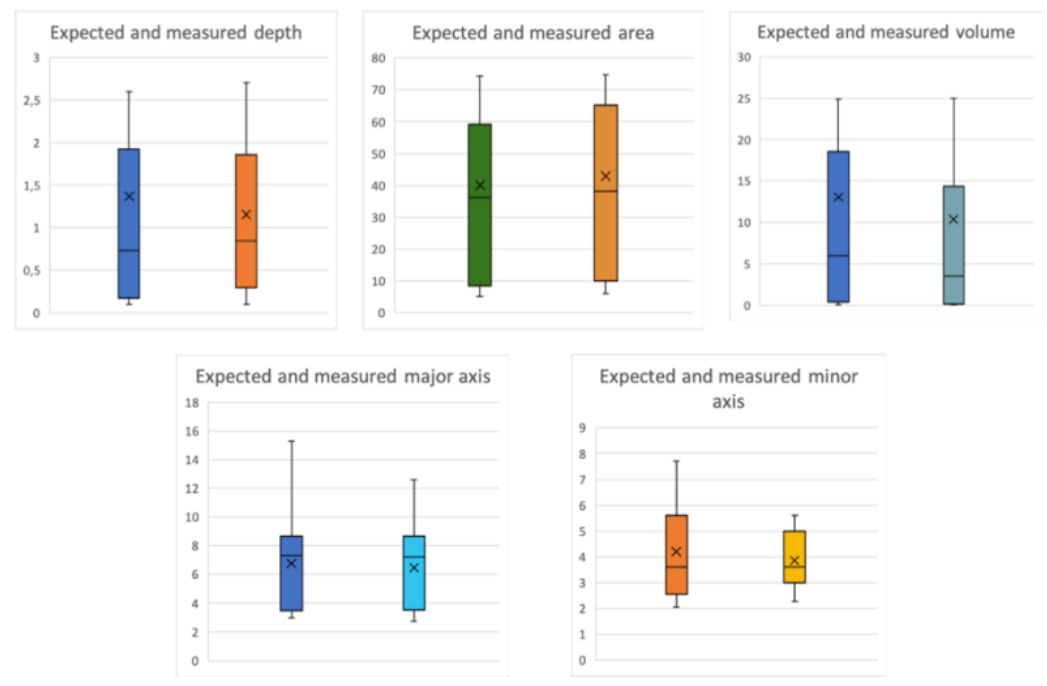

Figure 43: boxplot of the five components (expected vs. measured) 


\subsubsection{Discussion}

By automatically combing the information from high resolution photograph and 3D meshes, we were able to achieve very close results to those retrieved manually using Blender, which requires time and software manipulation expertise. Unfortunately, healthcare professionals and caregivers generally lack these two requirements. Hence, this system will enable them to automatically and non-intrusively retrieve the measurements of the pressure injuries and eventually track their healing evolution.

For the segmentation part, the state-of-the-art contributions were largely achieved using classical image processing and machine learning techniques. Since the databases used differ from one to another, as well as the number of images used, a comparison between the techniques could be misleading. Moreover, many researchers present their segmentation results using the accuracy. This metric is highly misleading as a high value of pixel accuracy does not always imply superior segmentation ability. The following contributions in the literature have stated their recall and Dice coefficient scores, which are more reliable metrics. Dhane et al. (Dhane 2016) used 105 Lower extremity wound images, preprocessed them using color correction and denoising and applied spectral clustering for wound segmentation, and applied morphological post-processing. The spectral clustering showed an average sensitivity 89.54\%. Veredas et al. (Veredas 2015) used 113 images of pressure injuries for training and 322 for testing, applied statistical color models from a Bayesian perspective to estimate the probability of a pixel to belong to the ROI and achieved an F-score of 73.89\%. Wannous et al. (Wannous 2011) used 50 images of all types of wounds and reached an average overlap score of $73.1 \%$. 
It is to be noted that the measurement of the volume in our system is relevant when the pressure injury is on a concave surface which can be closed without altering the shape of the wound. For instance, on Figure 40, both pressure injuries on the left and right are on the hip bone of the patient. The femur bone is above the surface, which creates a concave surface inside the wound. The pressure injury in the middle is located on the heel and ankle. As this surface is concave too, the center of the pressure injury will be located above the surface containing the boundaries of the wound. Hence, summing all tetrahedra bounded by the origin and a mesh triangle will result in adding more subvolumes inside the concave surface, which do not originally belong to the wound. Consequently, the result will be erroneous.
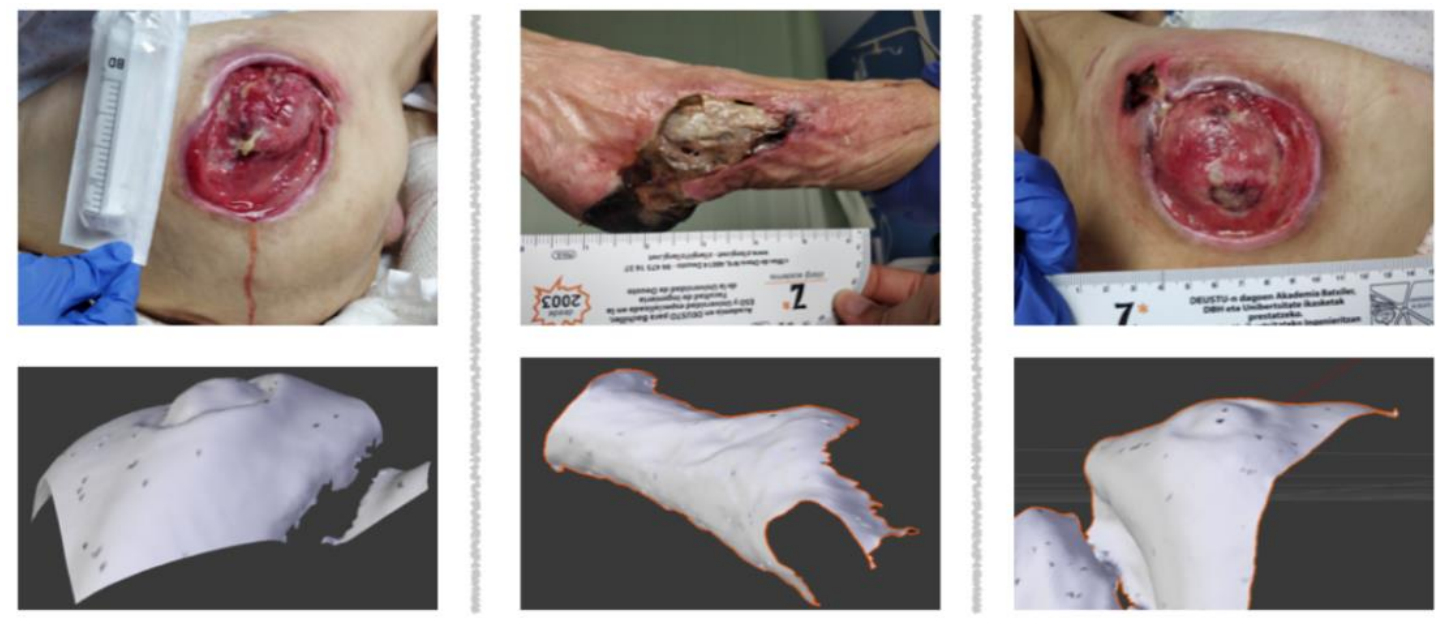

Figure 44: Example of pressure injuries invalid for the measurement of the volume: Left and right columns present two pressure injuries located on the hip bone, whereas the middle column presents a pressure injury located on the heel and ankle

Despite the fact that the segmentation precision was solely $87 \%$, which is mainly due to the ambiguous borders of some pressure injuries, the matching block slightly enlarges the borders of the mask in order to ensure that the whole wound is considered for the measurement. The ruler can also be detected in case the 3D mesh is not provided. 
This way, the area and diameters of the wound could be measured too. This part will be added to our proposed framework, that is currently being integrated in an interactive system with a user-friendly interface, which will be used during the future hospital study visits. This way, we will enlarge the database and reinforce the learning of our system.

Most hospitals require documentation of the pressure injury using images, and additionally, expect the healthcare professionals to take more detailed information. Using this system, they will have the ability to use the time with the patient more effectively. In order to independently use this system, medical centers will need to acquire the Structure Sensor, along with the mobile device to mount it on. The total acquisition time could range from 1 to $2 \mathrm{~min}$, for both the 2D image and 3D mesh combined. Our experience with caregivers during study visits demonstrated that they can capture the 3D meshes efficiently. The data captured will then be processed using the user-interface integrated within the device. Some training for the acquisition of the 3D meshes will be given. Hence, we will provide both a document and a video explaining the process.

\subsection{Chapter Summary}

In this chapter, we have portrayed our second and main contribution in the study of pressure injuries assessment using Deep Learning and imaging techniques. In the proposed framework, two main parts are combined. The first part addresses pressure injury segmentation where we used a pretrained Mask RCNN model which achieved a mean sensitivity score of 0.85 and a mean precision score of 0.87 for the segmentation. The 
binary mask predicted by the model is then used in the second part of the framework for wound's measurement. The 2D photograph used in the segmentation pipeline was matched with its corresponding 3D mesh in order to retrieve the vertices and faces corresponding to the wound in the 3D mesh. Then a series of operations were applied in order to extract all the necessary measurements: volume, depth, area and major and minor axes. The obtained measurements were compared to the ground-truth measurements which were obtained manually using Blender software confirmed the reliability of the proposed methodology. This system will enable physicians and care givers to optimally and non-intrusively assess pressure injuries. 


\section{CHAPTER 5}

\section{WEB-BASED APPLICATION}

This chapter presents the integration of some of the developed algorithms in a web-based application, which will be used by physicians and care givers, hopefully in the region of Bilbao in the Basque country. In this project, a device was previously developed in order to acquire data from the wounds, using different types of sensors and cameras. The data was then available in a web application so that the user could visualize the data captured during each test, and track the presence of the infection in the wound. The former analysis was solely made using the data from sensors (Alcohol, CO2, Ammonia and Acetone detectors). The contribution which was added to the existing project is the integration of imaging techniques by processing the images acquired during the tests from the two cameras available in the device, and delivering essential information for the assessment of pressure injuries, such the presence of each tissue type and their percentage in the wound, the measurements of the area and perimeter, etc. 
The following subsections will be presented as follows: a presentation of the tissue types segmentation models which were included in the web application, a brief introduction of the device used for acquisition, the improvements which were added in the wed application and a final discussion and conclusion.

\subsection{Segmentation models}

In this web application, four different models have been included to conduct the segmentation task: One model which segments the wound and the ruler, one for granulation tissue, one for slough tissue and one for necrotic tissue.

For the ground-truth annotations, an online annotator has been used to segment the desired ROIs and exports a JSON file with the corresponding areas and classes, as shown in figure 45.

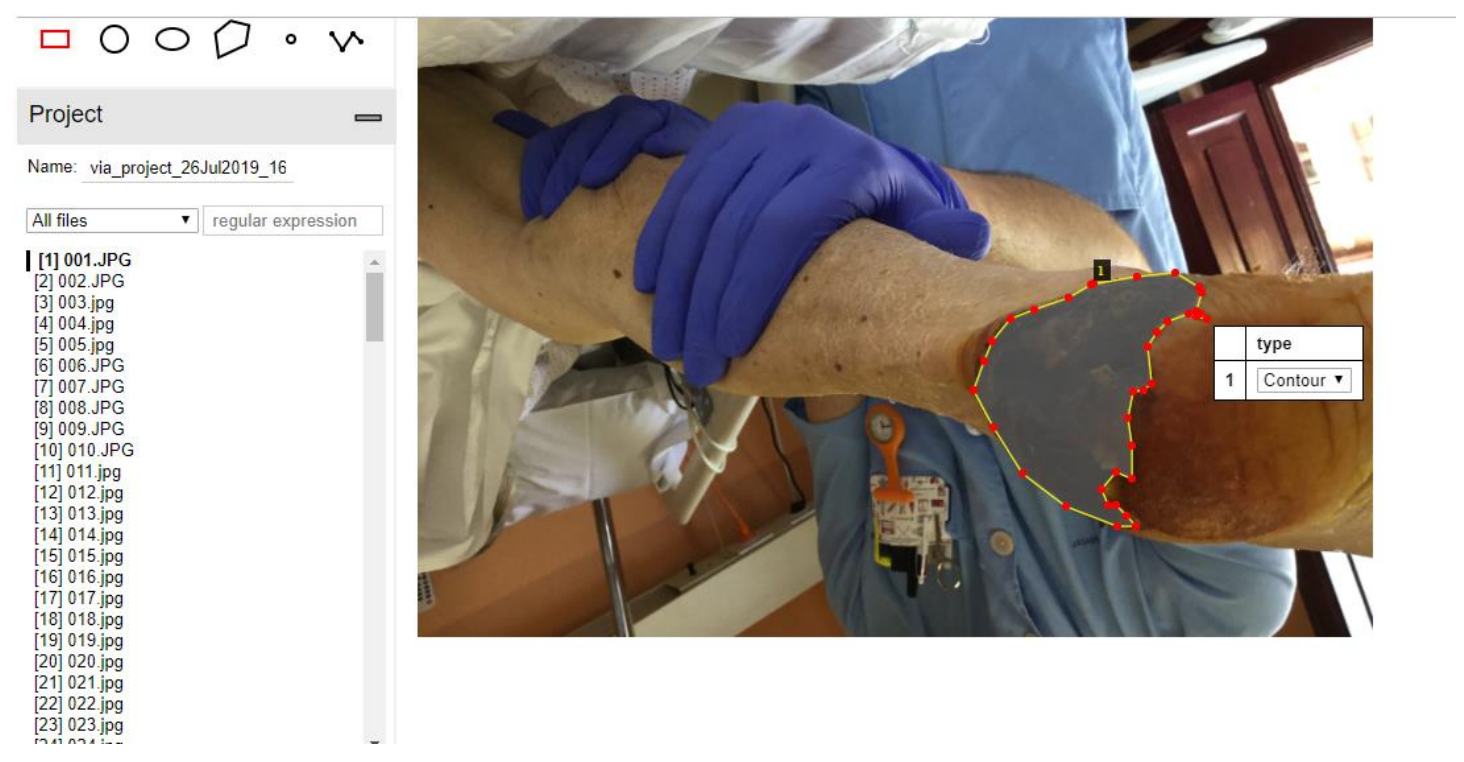

Figure 45: Image annotation illustration for necrotic tissue

As discussed in chapter 4, section 4.4.2, a Mask RCNN architecture was used to conduct the segmentation, where the best performance was obtained using a ResNet50 backbone with a mean Dice score of 0.83 for wound segmentation and 0.80 for the ruler, a mean 
sensitivity score of 0.85 for the wound and 0.83 for the ruler, and a mean precision score of 0.87 for the wound and 0.83 for the ruler, as shown in figure 46 .

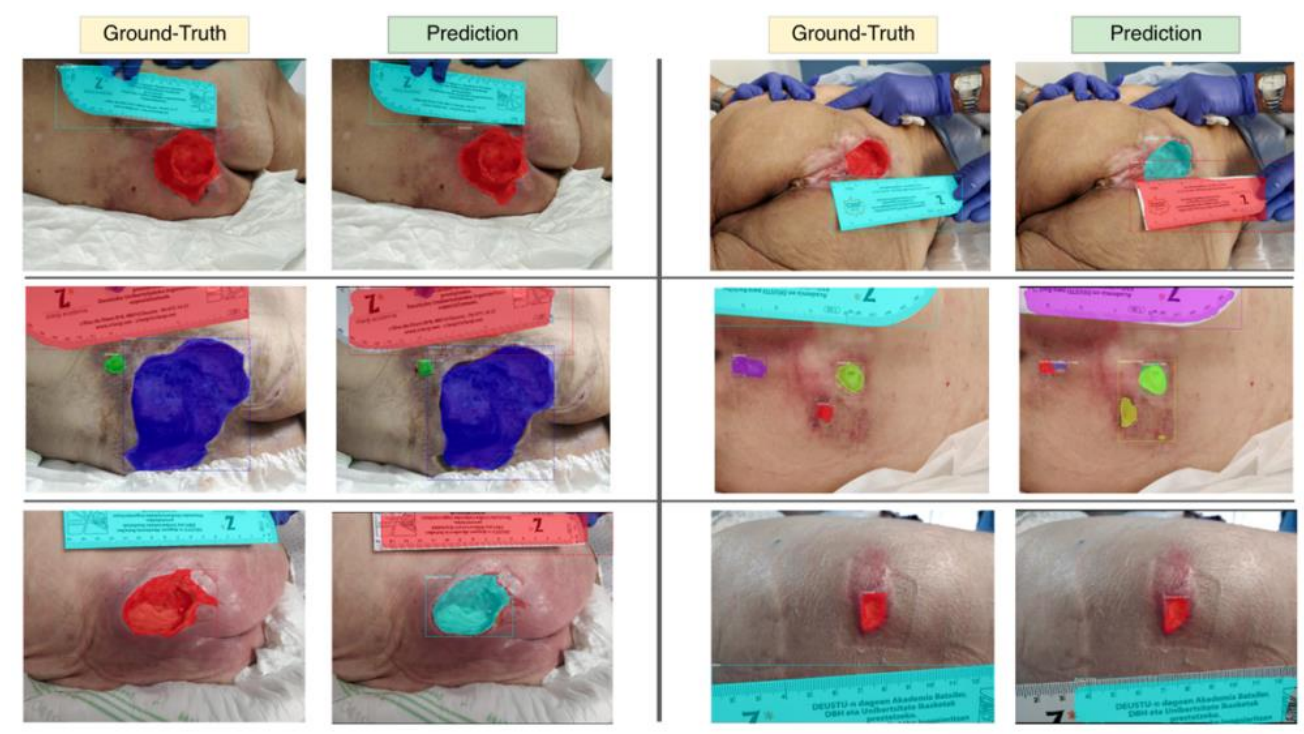

Figure 46: Results of the segmentation of pressure injuries and rulers

As for the granulation tissue, there was a total of 136 images which were containing granulation tissue (97 were used for training and 39 for testing). The mean DICE score was $88.6 \%$ and the mean precision of $93 \%$. For the slough tissue, there was a total of 144 images which were containing slough tissue (115 were used for training and 29 for testing). The mean DICE score was $83.3 \%$ and the mean precision of $86 \%$. As for necrotic tissue, there was a total of 84 images which were containing necrotic tissue (77 were used for training and 7 for testing). The mean DICE score was $80.1 \%$ and the mean precision of $84 \%$. These results seem lower than the ones presented in chapter 3 with the proposed segmentation model. This is because our first proposed model was applied on images which were already segmented and only the wound was kept. However, the Mask RCNN takes the whole image, with makes it a more challenging task. 


\subsection{The device}

The main purpose of the device is to non-intrusively acquire data from the wounds in order to detect if the bacteria is present in the pressure injury. Different sensors were included in the device, such as analog sensors (MQ3 for alcohol, the MQ135 for CO2 and the MQ138 for acetone and TGS286 for ammonia), temperature sensor (DHT22), Distance sensor (HC-SR04), and two optical cameras and one thermal camera, as shown in fig. 47.

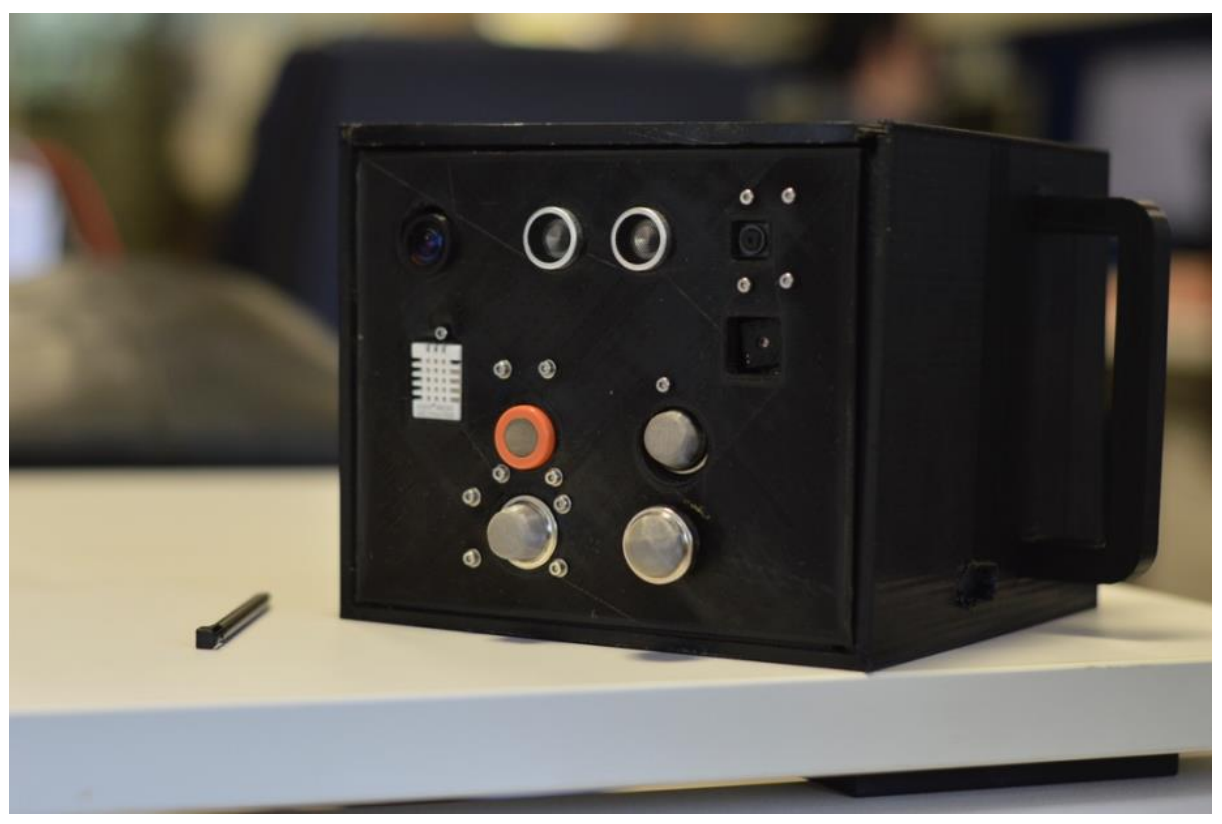

Figure 47: The device for pressure injury data acquisition

In the front face, a touchscreen with a touch pen is used for user interaction. The 5inch HDMI LCD by Waveshare is connected with the raspberry pi through HDMI for the display itself and then with SPI for the touch functionality.

The user interface in the device start with a login window as shown in fig. 48. For an easier user authentication, a pin number associated to each doctor could be entered using number buttons. 


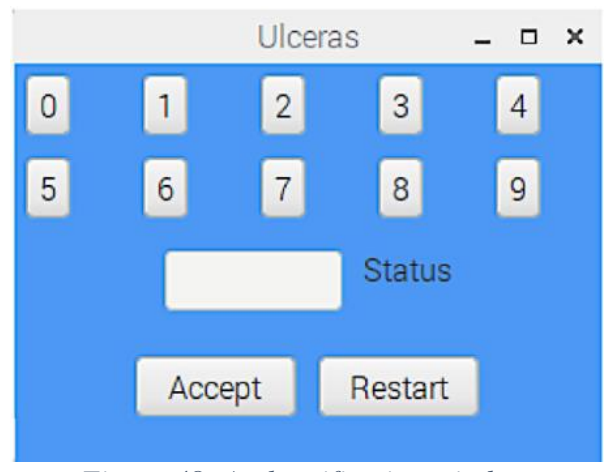

Figure 48: Authentification window

After a successful log in, the next widow enables the selection of the patient which we want to scan. In case of an available internet connection, the doctor could also access the past test. The doctor can start a new test by clicking on "New Test" tab, as shown in fig. 49.

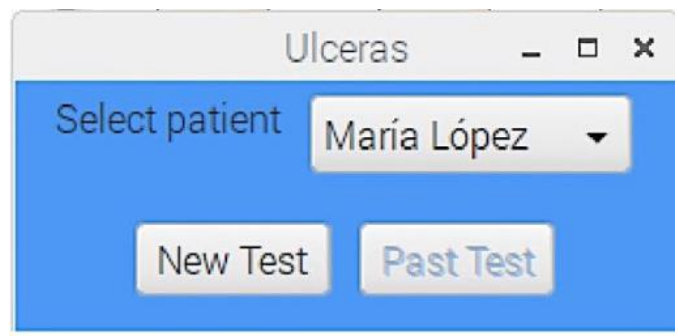

Figure 49: Patient selection window

If the doctor chooses to access a previous test, he/she can either upload it to the web server in case it hasn't been done, or delete them, as shown in fig. 50.

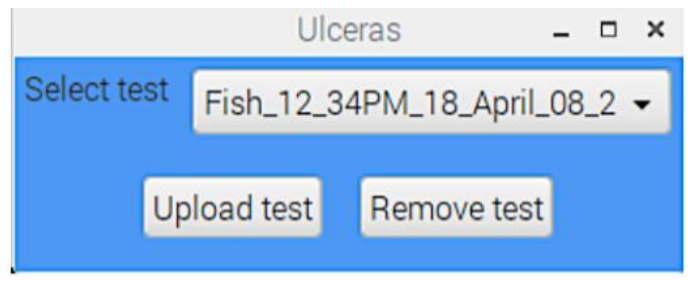

Figure 50: Past tests window

If the doctor chooses to make a new test, then a test window opens as shown in fig. 51 . Three different buttons are available, each one for a different reading: the gas sensors, the temperature with the thermal camera and the photo for the cameras. In case the doctor wants to repeat the test, he/she can push the restart button and all the current data will be 
removed. In case of an available Internet connection, the doctor can upload the data in the web server by pushing the button "Upload".

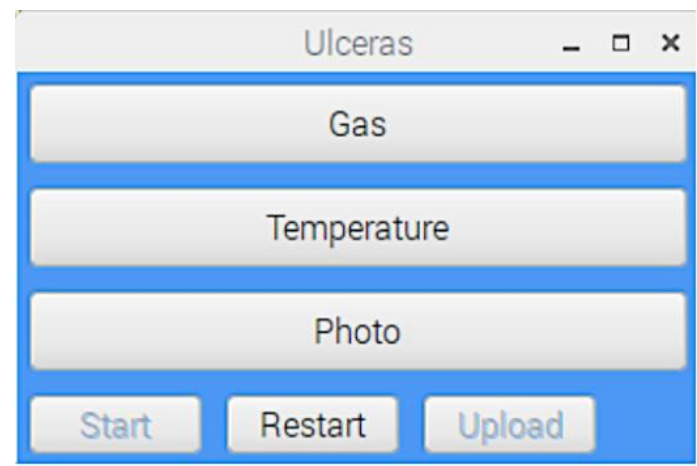

Figure 51: Test window

When the user chooses to acquire the Gas data, a gas window is displayed and four different boxes corresponding to the four sensors show the real-time gas values.

Once the test is finished, a graph is displayed with the concentrations of each sensor through the test, as shown in fig. 52.
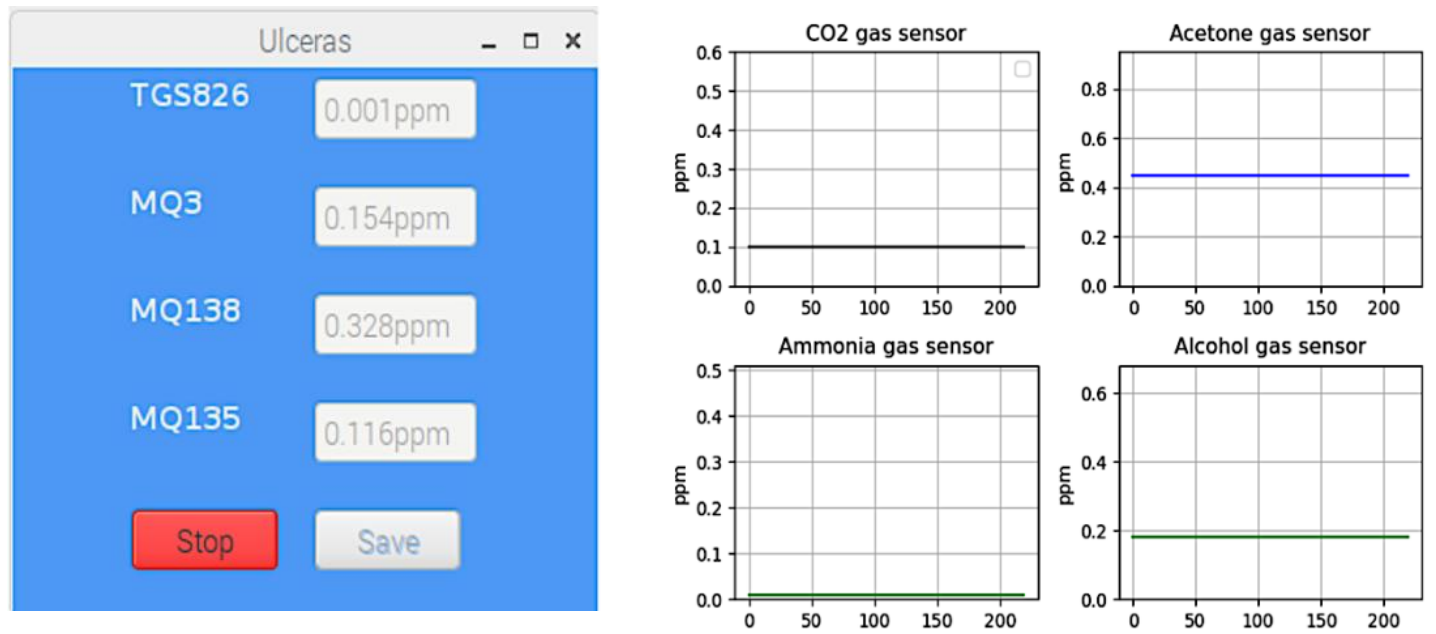

Figure 52: Gas window and information display

When the user chooses to capture the temperature using the temperature camera, a window displays the captured image, with a possibility to retake the image as shown in fig. 53. 


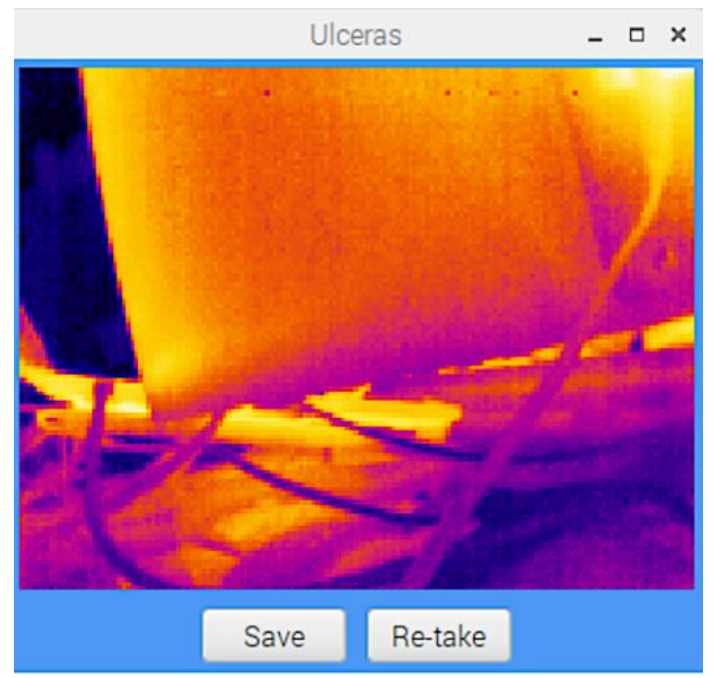

Figure 53: Temperature window

And lastly, when the doctor wants to capture images of the wound using both of the cameras, similarly to the temperature camera, he/she can take the picture or retake it in case he/she wants to redo the capturing, as shown in fig. 54.

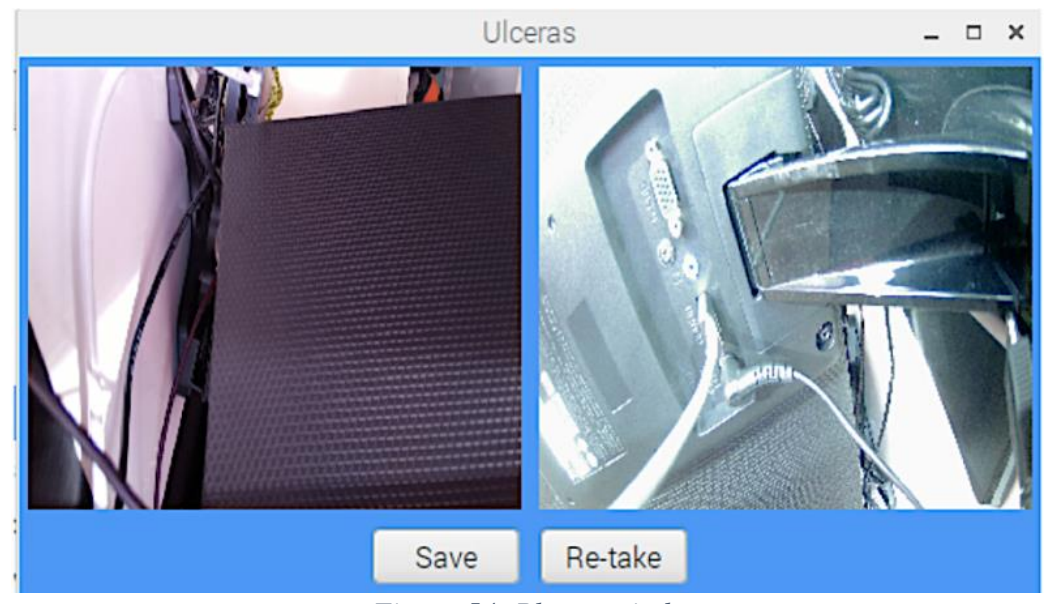

Figure 54: Photos window

\subsection{Web server}

The web server application is developed by using Django, using a template named Gentelella developed by Colorlib. Django has the advantage of an easy setup of the database, as it has an integration through the Django models, which are defined and then 
migrated to the database. The developed web server application contains the following main views:

Login and Register: used for logging in and registering to the web. The doctor needs to enter his/her credentials, username and password to login, for the registration the necessary details are; name, last name, username email and password, as shown in figure 55.

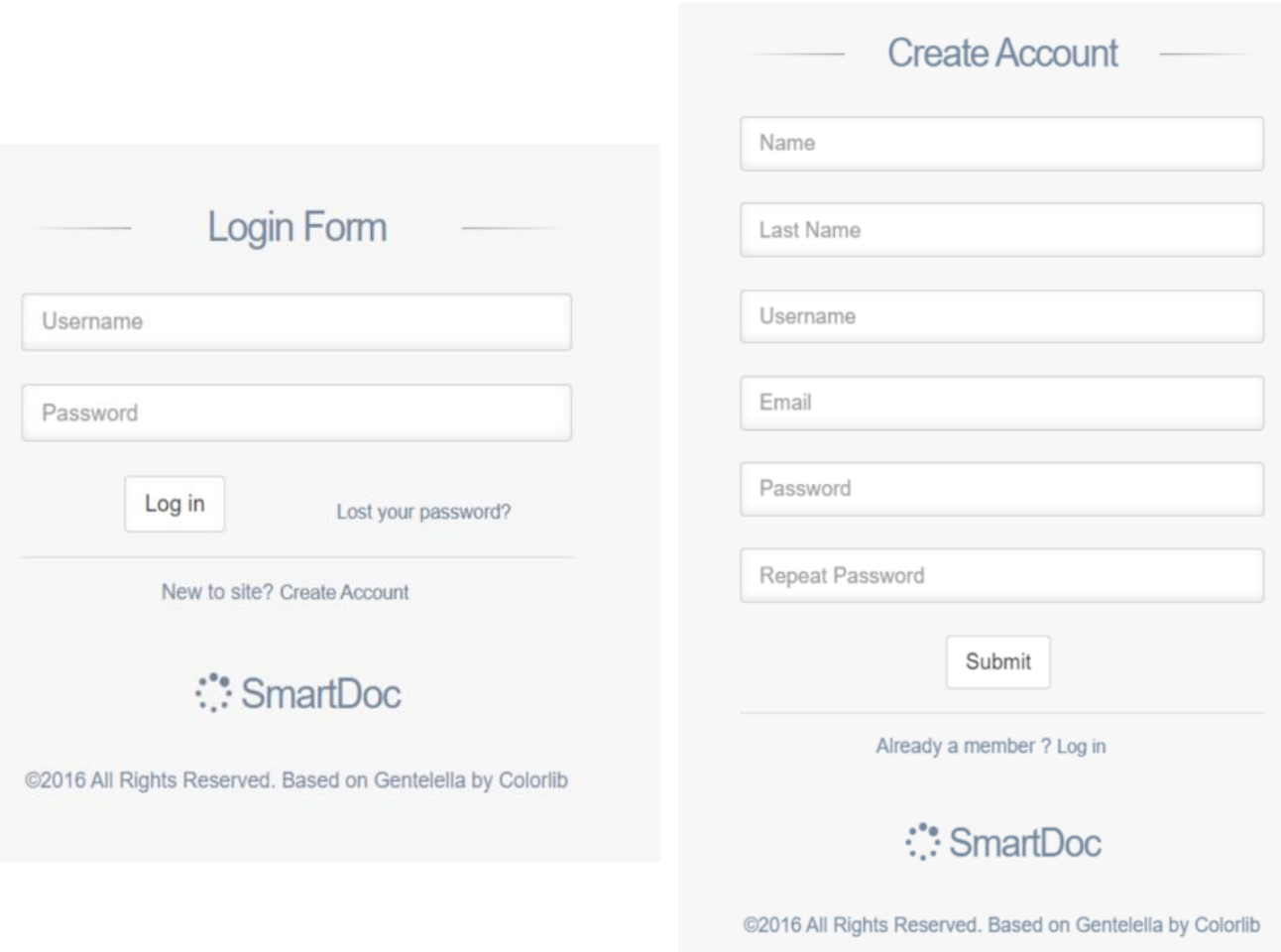

Figure 55: Log in and registration

Once the doctor has successfully logged in, a page with a list of his/her patients will open, showing the user photo if existing, the name and id, as shown in figure 56. 


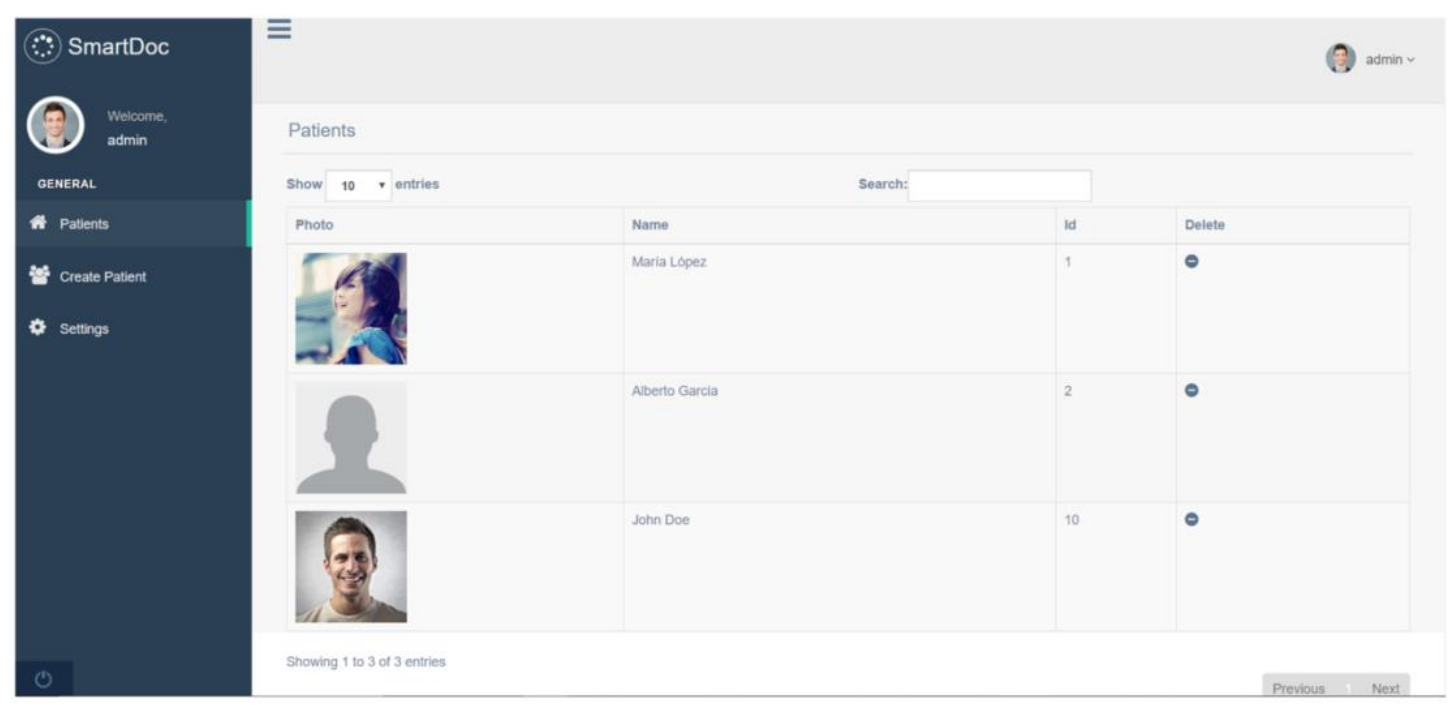

Figure 56: Patients list overview

Once a patient is chosen, a page of all the tests made for that patient will be shown. Previously, solely information about the infection prediction from the gas sensor data was shown in a table. The integration of the image processing results is evidenced by the different columns added, as well as an evolution graph of the evolution of different tissue type percentages in the wound, as shown in figure 57. Each one of the table columns contains a specific result:

- Right image: when "image" is clicked, the right image is shown with the wound and ruler segmented, as shown if figure 58.

- Left image: Similarly, when "image" is clicked, the left image is shown with the wound and ruler segmented

- Number of PIs: This column contains the number of detected PIs in the image.

- Tissue types: Contains the detected tissue types in the wound.

- Ruler: If there is a detected ruler in the image, the value is "Yes". 
- Perimeter/Area: contain the measurements of the perimeter and area $\left(\mathrm{cm} / \mathrm{cm}^{2}\right)$, after the doctor selects $1 \mathrm{~cm}$ distance from the image (currently being developed).

- Granulation/Slough/Necrosis: These columns contain the percentage of each of one of the detected tissue types, and when the percentage if clicked on, an image of the wound with the corresponding segmented tissue type is shown.

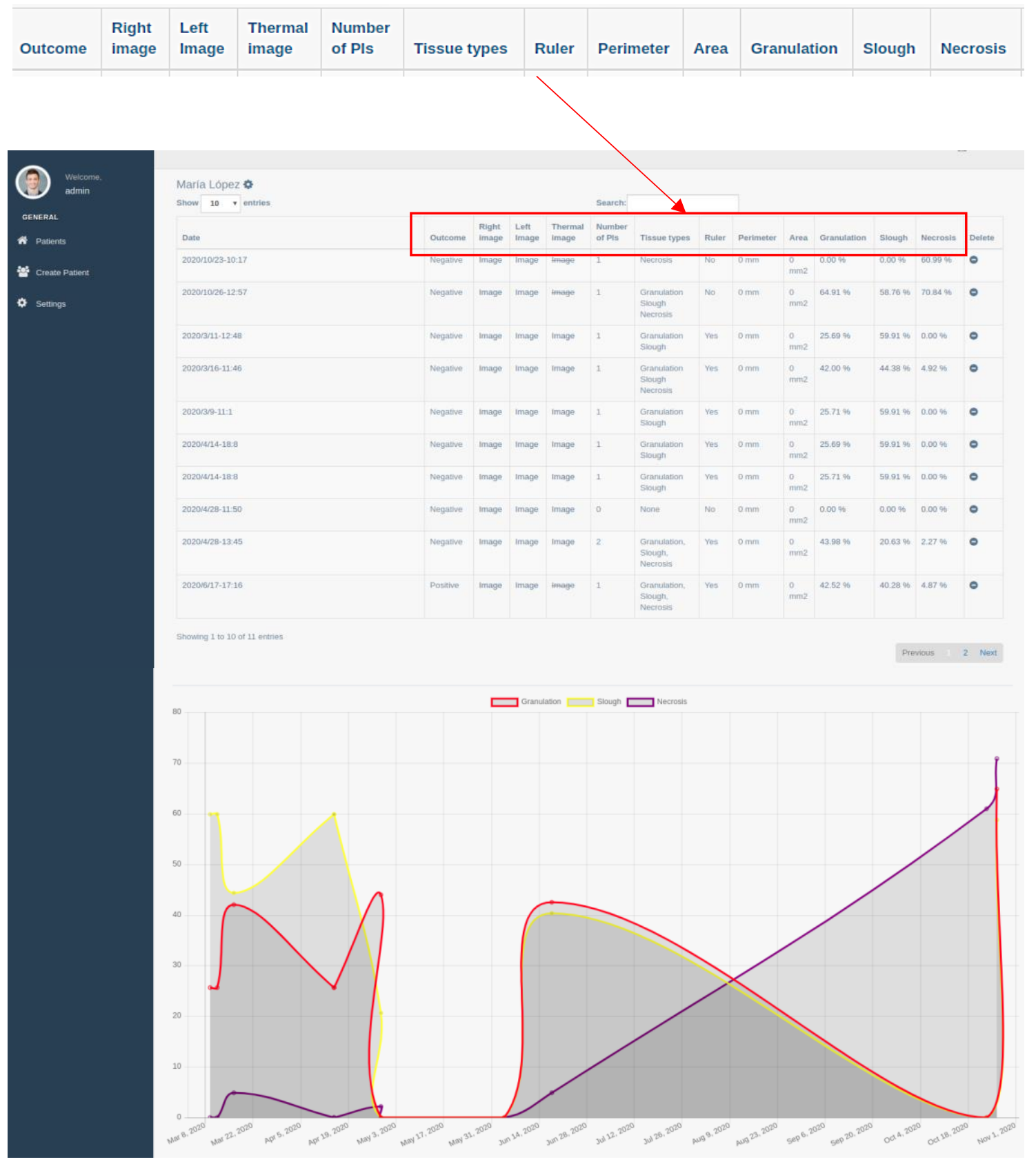

Figure 57: Specific patient's tests overview 


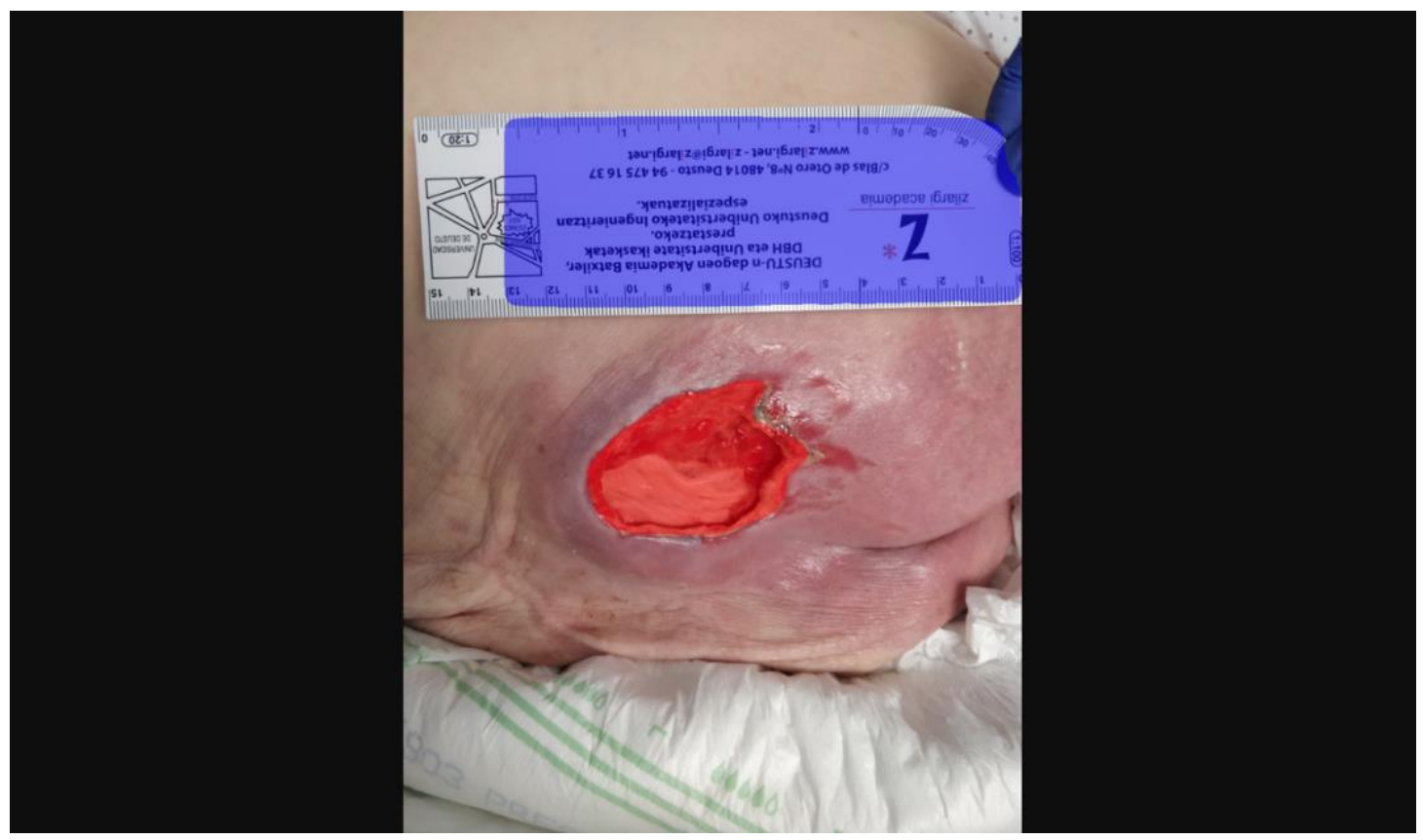

Figure 58: Illustration of image segmentation when "right image" is clicked on

If the doctor selects a specific test date, a new page opens and shows four graphs of the collected gas data, the captured images, the distance from which it was captured from, a table of the findings previously mentioned, a pie chart with the percentages of the three different tissue types, and a selection of segmented images of the detected tissue types, as shown in figure 59. A color panel has been chosen for an easier visualization of the segmentations: red for the wound, blue for the ruler (for the whole image segmentation), then for specific tissue types, red was chosen for granulation, yellow for slough and purple for necrosis, as shown in figure 60.

Once the doctor has edited the different segmentations which he/she does not agree with, the edited segmentation will be saved into the database and the results of that test will be updated accordingly, as shown in figure 61. 


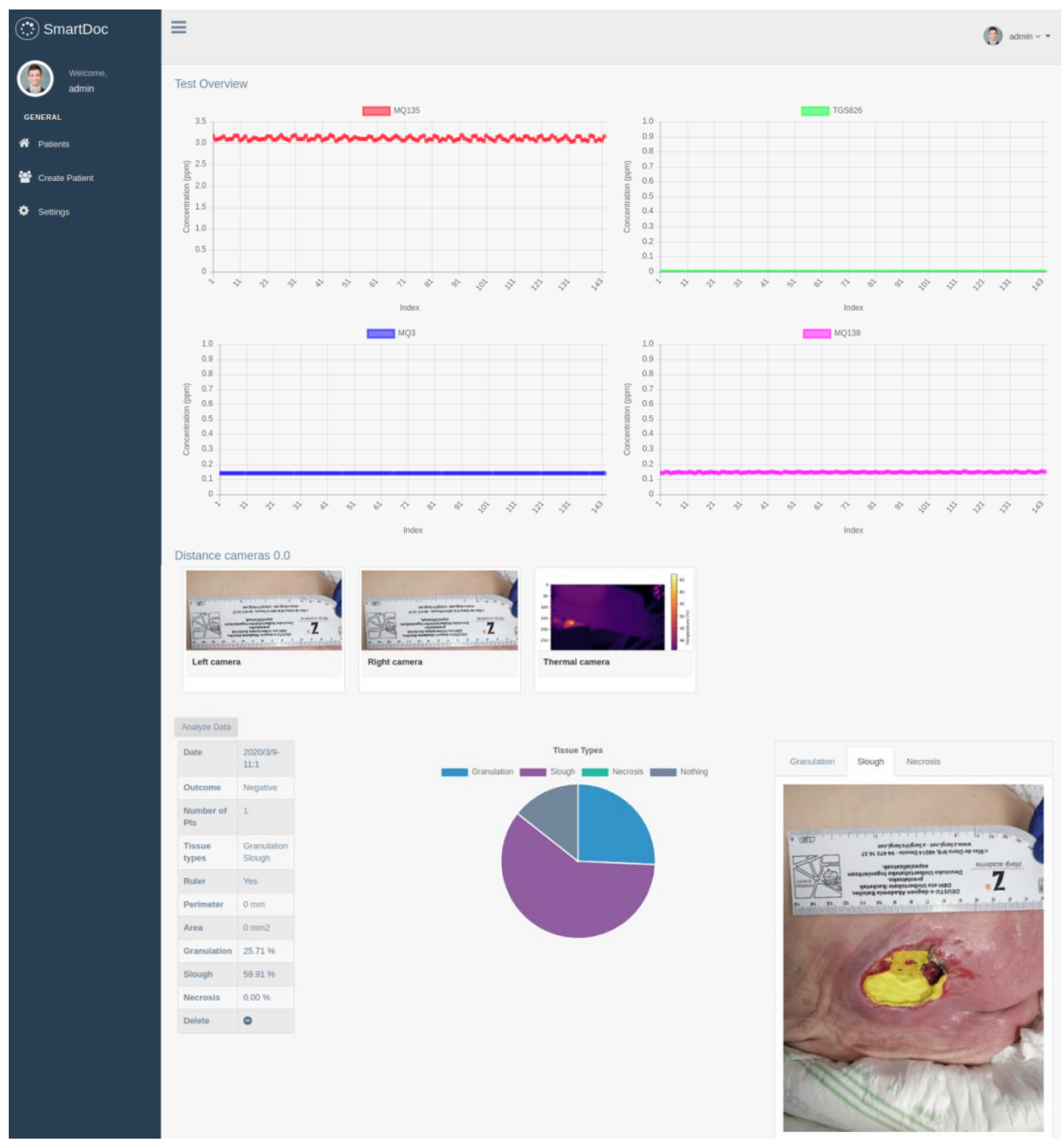

Figure 59: Specific test's page view
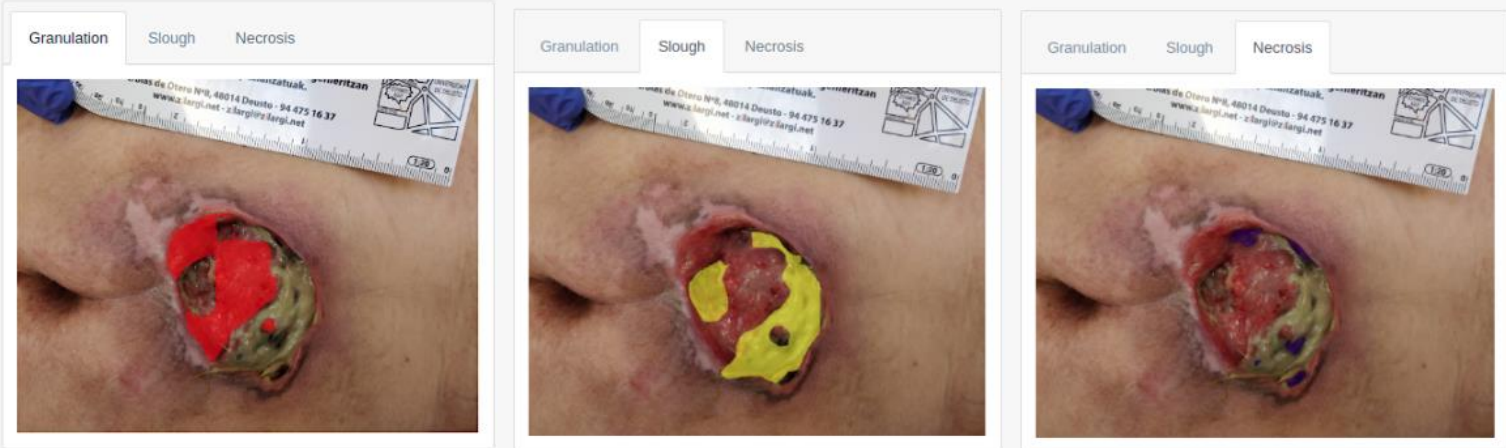

Figure 60: Three tissue types segmentation views 


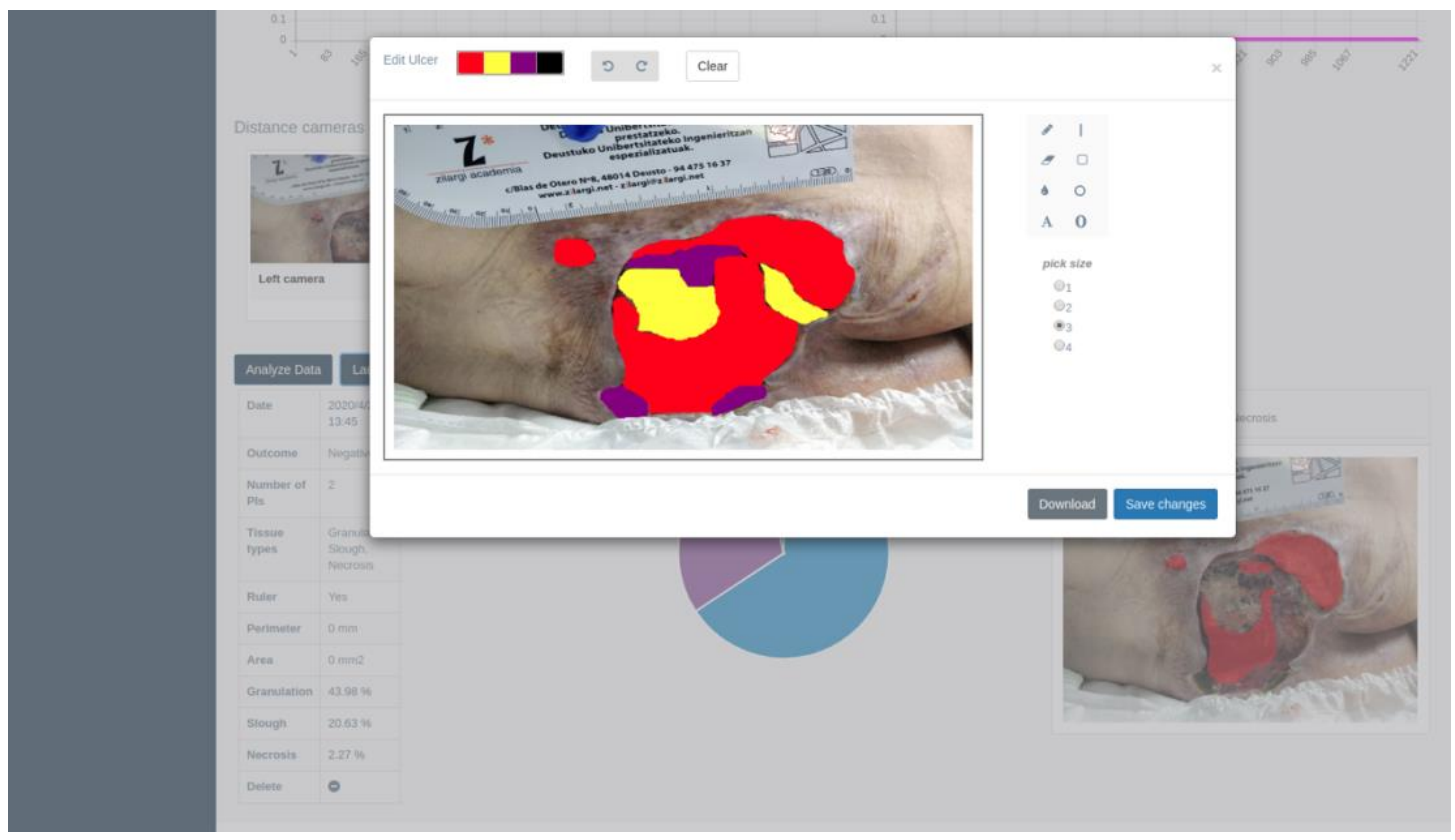

Figure 61: Segmentation editing window

The current improvement of this web application is the inclusion of an interactive window which will enable the doctor to edit the segmentations and insert the distance of $1 \mathrm{~cm}$ in case the ruler is present, so that the algorithm could directly provide the real-world measurement of the wound.

\subsection{Chapter summary}

In this chapter, we have presented our integration of pressure injury imaging algorithms in a web-based application for an easier and non-intrusive assessment of pressure injuries. This application in connected to a sensor device, which was previously developed by a Masters student in the university of Deusto, Spain.

In this application, the doctor can retrieve the data which was acquired using the device and apply the algorithms of detection and segmentation. The output results retrieved from the acquired images are the detection and segmentation of the wound and the ruler if 
present, the segmentation of the three tissue types and the calculation of their percentages in the wound. Using the ruler, the perimeter and area can also be measured. If the models' segmentations are not very precise, an interactive user interface for editing enables the doctor to rectify the segmentations, and the measurements are then updated automatically. 


\section{CHAPTER 6}

\section{CONCLUSION AND FUTURE WORK}

This dissertation studied new approaches of Deep Learning to tackle one of the important health issues in the medical sector using imaging techniques. It revolves around the assessment of pressure injuries which represent a serious health issue especially in elderly and bed-bound individuals. Using 2D and 3D imaging techniques, along with Deep Learning technology, the objectives which were set in the beginning of the thesis were successfully met.

This dissertation work has provided several contributions in medical imaging in the form of end-to-end frameworks, including preprocessing of the data, the design of Deep Learning architectures suitable for each type of data and reliable results using typical validation metrics of each application. All the three main specific objectives of this dissertation were fulfilled during the research process:

SO1: Construction of the database containing 2D photographs and 3D meshes of pressure injuries: This objective was successfully completed by collaborating with 
several hospitals in the region of Bilbao (Cruces Hospital, Santa Marina Hospital, Basurto Hospital) and doing hospital visits during a period of 24 months in order to acquire the data necessary to conduct the research, in addition to personal visits to patients houses. The database was finally composed of 210 photographs of pressure injuries, where 35 has a corresponding 3D mesh.

SO2: To define the current state of the art achievements in pressure injury imaging systems. This objective was successfully completed by the publication of an article that consisted of an analysis of wound imaging techniques with artificial intelligence, offering a literary review combined with the novelty of Deep Learning techniques to tackle this medical application.

SO3: To design and implement algorithms for the segmentation, classification and measurement of pressure injuries: This objective which represents the core part of this study case was fulfilled by designing two end-to-end systems. The first proposed system is based on a Convolutional Neural Network $(\mathrm{CNN})$ devoted to performing optimized segmentation of the different tissue types present in pressure injuries (granulation, slough, and necrotic tissues). The obtained results showed an overall average classification accuracy of $92.01 \%$, an average total weighted Dice Similarity Coefficient of $91.38 \%$, and an average precision per class of $97.31 \%$ for granulation tissue, $96.59 \%$ for necrotic tissue, and $77.90 \%$ for slough tissue. The second proposed system automatically segments and measures all the quantitative characteristics of pressure injuries using their $2 \mathrm{D}$ and $3 \mathrm{D}$ information. Then, the developed algorithms for segmentation were integrated in a webbased application in order to enable the doctors to track the healing of the wound by 
following the evolution of the tissue types inside the wound, its measurements and infection.

From the obtained results which were presented in the previous chapters, we can state that use of Deep Learning can definitely take medical decisions to the next level, improving the quality of diagnosis of several heath issues by providing reliable results to care givers from the analysis of patients' data. The motivation for conducting this research in some of the crucial medical issues that we are facing in our society nowadays arises from the eagerness to take medical research to a higher level in order to alleviate the severity of such health issues because of a lack of prevention or assessment tools. Meeting with physicians and caregiver has not only helped us to better conduct this research, but also confirmed to us the necessity of such novel methods to deal with these health issues.

As a result of these findings, three papers were published in top-tier international journals. The first paper, as shown in Table 4, introduces the state the art literature survey, where 114 relevant articles in the field of would image analysis using classical machine learning and recent Deep Learning techniques were analyzed.

Table 4:Details of the publication (Zahia 2020b)

\begin{tabular}{|c|c|}
\hline Title & $\begin{array}{l}\text { Pressure injury image analysis with machine learning techniques: } \\
\text { A systematic review on previous and possible future methods }\end{array}$ \\
\hline Authors & $\begin{array}{l}\text { Sofia Zahia, Maria Begoña Garcia Zapirain, Xavier Sevillano, } \\
\text { Alejandro González, Paul J Kim, Adel Elmaghraby }\end{array}$ \\
\hline Journal & Artificial Intelligence in Medicine \\
\hline Date & 18 October 2019 \\
\hline Impact Factor & $4.383(2019)$ \\
\hline DOI & https://doi.org/10.1016/j.artmed.2019.101742 \\
\hline
\end{tabular}


The second paper, as shown in Table 5, presents a novel contribution in tissue type segmentation and classification using Convolutional Neural Networks.

Table 5: Details of the publication (Zahia 2018)

\begin{tabular}{|l|l|}
\hline Title & $\begin{array}{l}\text { Tissue classification and segmentation of pressure injuries using } \\
\text { convolutional neural networks }\end{array}$ \\
\hline Authors & $\begin{array}{l}\text { Sofia Zahia, Daniel Sierra-Sosa, Begonya Garcia-Zapirain, Adel } \\
\text { Elmaghraby }\end{array}$ \\
\hline Journal & Computer Methods and Programs in Biomedicine \\
\hline Date & 22 February 2018 \\
\hline Impact Factor & $3.632(2019)$ \\
\hline DOI & https://doi.org/10.1016/j.cmpb.2018.02.018 \\
\hline
\end{tabular}

As for the third paper, as shown in Table 6, and end-to-end system for a full assessment of pressure injuries using 2D photographs and 3D meshes of the wounds along with Deep Learning and computer vision techniques was presented.

Table 6: Details of the publication (Zahia 2020)

\begin{tabular}{|c|c|}
\hline Title & $\begin{array}{l}\text { Integrating 3D Model Representation for an Accurate Non-Invasive } \\
\text { Assessment of Pressure Injuries with Deep Learning }\end{array}$ \\
\hline Authors & Sofia Zahia, Begonya Garcia-Zapirain, Adel Elmaghraby \\
\hline Journal & Sensors \\
\hline Date & 21 May 2020 \\
\hline Impact Factor & $3.275(2019)$ \\
\hline DOI & $10.3390 / \mathrm{s} 20102933$ \\
\hline
\end{tabular}

While this study adds to the existing body of literature on the potential of Deep Learning for tackling different medical applications, there are some limitations to the study that should be considered. The efficiency of Deep Learning models mostly relies on the quality of the dataset used for the learning. The database used for the study was acquired 
during several study visits. However, due to the tight timetable of physicians and nurses, the collected database can't be considered big enough to provide generalizable results. Although the obtained results are very satisfactory, a bigger dataset would definitely enhance the generalizability of the trained models.

During the process of data collection, as we were dealing with pressure injuries of stage 3 and 4, many patients passed away after our first visits. Unfortunately, we were unable to add the part of the healing prediction as we couldn't get enough data from the same patients through a long duration. This part of the study, as a future step, would represent a significant contribution in the assessment of pressure injuries, as it would help doctors and caregivers to predict the healing of the wound based on the prescribed treatment and the evolution of the wound. 


\section{REFERENCES}

(Abadi 2016) Abadi, M.; Agarwal, A.; Barham, P.; Brevdo, E.; Chen, Z.; Citro, C.;

Corrado, G.S.; Davis, A.; Dean, J.; Devin, M.; et al. Tensorflow: Largescale machine learning on heterogeneous distributed systems. arXiv 2016, 1603, 04467.

(Akil 2020) Akil, M., Saouli, R., \& Kachouri, R. (2020). Fully automatic brain tumor segmentation with Deep Learningbased selective attention using overlapping patches and multi-class weighted cross-entropy. Medical Image Analysis, 101692.

(Albouy 2007) Albouy B, Lucas Y, Treuillet S. 3D modeling from uncalibrated color images for a complete wound assessment tool. Proc 29th annual international conference of the IEEE engineering in medicine and biology society (EMBS) 2007:33233326.

(Amoon 2020) Amoon, M., Altameem, T., \& Altameem, A. (2020). Internet of things Sensor Assisted Security and Quality Analysis for Health Care Data Sets Using Artificial Intelligent Based Heuristic Health Management System. Measurement, 107861. 
(Avis 1997) Avis, D.; Bremner, D.; Seidel, R. How good are convex hull algorithms?

Comput. Geom. 1997, 7, 265-301.

(Bishop 1986) Bishop, G.; Weimer, D.M. Fast phong shading. ACM SIGGRAPH

Comput. Graph. 1986, 20, 103-106.

(Bochko 2010) Bochko V, Valisuo P, Harju T, Alander J. Lower extremity ulcer image segmentation of visual and near infrared imagery. Skin Res Technol 2010;16(2):190-7.

(Bodini 2009) Bodini, B., \& Ciccarelli, O. (2009). Diffusion MRI in neurological disorders. In Diffusion MRI (pp. 175-203). Academic Press.

(Bon 2000) Bon FX, Briand E, Guichard S, Couturaud B, Revol M, Servant JM, et al. Quantitative and kinetic evolution of wound healing through image analysis. IEEE Trans Med Imaging 2000;19:767772.

(Bonabeau 1999) Bonabeau E, Dorigo M, Theraulaz G. Swarm intelligence: from natural to artificial systems. Oxford: Oxford University Press; 1999.

(Bovik 2010) A.C. Bovik, Handbook of Image and Video Processing, Academic press, 2010.

(Brown 2003) Brown G. Long-term outcomes of full-thickness pressure ulcers: healing and mortality. Ostomy Wound Manag 2003;49(10):42-50

(Bulstrode 1986) Bulstrode CJK, Goode AW, Scott PJ. Stereophotogrammetry for measuring rates of cutaneous healing: a comparison with conventional techniques. Clin Sci 1986;71:437443.

(Chakraborty 2015) Chakraborty C, Gupta B, Ghosh SK. Chronic wound tissue characterization under telemedicine framework. 2015 17th international 
conference on e-health networking, application and services (HealthCom). 2015 October. p. 569-73.

(Chakraborty 2016) Chakraborty C, Gupta B, Ghosh SK, Das DK, Chakraborty C. Telemedicine supported chronic wound tissue prediction using classification approaches. J Med Syst 2016;40(3):68.

(Chakraborty 2017) Chakraborty C. Chronic wound image analysis by particle swarm optimization technique for tele-wound network. Wirel Pers Commun 2017;96(3):3655-71.

(Chang 2017) Chang, H., Han, J., Zhong, C., Snijders, A., Mao, J.-H., Jan. 2017. Unsupervised transfer learning via multiscale convolutional sparse coding for biomedical applications. IEEE transactions on pattern analysis and machine intelligence.

(Chaves 2015) Chaves MEA, Silva FSd, Soares VPC, Ferreira RAM, Gomes FSL, Andrade RMd, et al. Evaluation of healing of pressure ulcers through thermography: a preliminary study. Res Biomed Eng 2015;31:39.

(Cheng 2018) Cheng, X., Zhang, L., \& Zheng, Y. (2018). Deep similarity learning for multimodal medical images. Computer Methods in Biomechanics and Biomedical Engineering: Imaging \& Visualization, 6(3), 248-252.

(Chollet 2015) Chollet, F. Keras: Deep Learning Library for Theano and Tensorflow. Available online: https://www.datasciencecentral.com/profiles/blogs/keras-deep-learninglibrary-for-theano-and-tensorflow 
(Conv)

https://leonardoaraujosantos.gitbooks.io/artificial-

\section{inteligence/content/convolutional_neural_networks.html}

(Cuddigan 2016) Cuddigan J. Clinical aspects of deep tissue pressure injury. 2016

(Dalya 2016) Dalya V, Shedge DK. Design of smartphone-based wound assessment system. International conference on automatic control and dynamic optimization techniques (ICACDOT). 2016 September. p. 709-12.

(Delode 2001) Delode J, Rosow E, Roth C, Adams J, Langevin F. A wound-healing monitoring system. ITBM-RBM 2001;22:4952.

(Dhane 2016) Dhane DM, Krishna V, Achar A, Bar C, Sanyal K, Chakraborty C. Spectral clustering for unsupervised segmentation of lower extremity wound beds using optical images. J Med Syst 2016;40:207.

(Dhane 2017) Dhane DM, Maity M, Mungle T, Bar C, Achar A, Kolekar M, et al. Fuzzy spectral clustering for automated delineation of chronic wound region using digital images. Comput Biol Med 2017;89:551-60.

(Dou 2015) Dou, Q., Chen, H., Yu, L., Shi, L., Wang, D., Mok, V. C., Heng, P. A., 2015. Automatic cerebral microbleeds detection from MR images via independent subspace analysis based hierarchical features. Conf Proc IEEE Eng Med Biol Soc, 7933-7936.

(Dou 2016b) Dou, Q., Chen, H., Yu, L., Qin, J., Heng, P. A., 2016b. Multi-level contextual 3D CNNs for false positive reduction in pulmonary nodule detection,

(EPUAP 1999) European Pressure Ulcer Advisory Panel (EPUAP). Guidelines on treatment of pressure ulcers. EPUAP Rev 1999;1:3133. 
(Fauzi 2015) Fauzi, M.F.A.; Khansa, I.; Catignani, K.; Gordillo, G.; Sen, C.K.; Gurcan, M.N. Computerized segmentation and measurement of chronic wound images. Comput. Biol. Med. 2015, 60, 74-85.

(Felzenszwalb 2004) Felzenszwalb PF, Huttenlocher DP. Efficient graph-based image segmentation. Int J Comput Vis 2004;59(2):167-81.

(Filko 2010) Filko D, Antonic D, Huljev D. WITA-Application for wound analysis and management. 2010 12th IEEE international conference on e-health networking applications and services (Healthcom). 2010. p. 68-73.

(Fischler 1081) Fischler, M.A.; Bolles, R.C. Random sample consensus: A paradigm for model fitting with applications to image analysis and automated cartography. Commun. ACM 1981, 24, 381-395.

(Garcia-Zapirain 2018) Garcia-Zapirain B, Sierra-Sosa D, Ortiz P, Isaza-Monsalve M, Elmaghraby A. Efficient use of mobile devices for quantification of pressure injury images. Technol Health Care 2018:1-12.

(Ghafoorian 2017) Ghafoorian, M., Karssemeijer, N., Heskes, T., Bergkamp, M., Wissink, J., Obels, J., Keizer, K., de Leeuw, F.-E., van Ginneken, B., Marchiori, E., Platel, B., 2017. Deep multi-scale location-aware 3d convolutional neural networks for automated detection of lacunes of presumed vascular origin. NeuroImage: Clinical.

(Gholami 2017) Gholami, P.; Ahmadi-pajouh, M.A.; Abolftahi, N.; Hamarneh, G.; Kayvanrad, M. Segmentation and measurement of chronic wounds for bioprinting. IEEE J. Biomed. Health Inf. 2017, 22, 1269-1277. 
(Girshick 2014) Girshick, R., Donahue, J., Darrell, T., \& Malik, J. (2014). Rich feature hierarchies for accurate object detection and semantic segmentation. In Proceedings of the IEEE conference on computer vision and pattern recognition (pp. 580-587).

(Girshick 2015) Girshick, R. (2015). Fast r-cnn. In Proceedings of the IEEE international conference on computer vision (pp. 1440-1448).

(Gulsun 2016) Gulsun, M. A., Funka-Lea, G., Sharma, P., Rapaka, S., Zheng, Y., 2016. Coronary centerline extraction via optimal flow paths and CNN path pruning. In: Med Image Comput Comput Assist Interv. Vol. 9902 of Lect Notes Comput Sci. Springer, pp. 317-325

(Haider 2016) Haider A, Alhashim M, Tavakolian K, Fazel-Rezai R. Computer-assisted image processing technique for tracking wound progress. 2016 IEEE international conference on electro information technology (EIT). 2016. p. $0750-4$.

(Hani 2011) Hani AFM, Arshad L, Malik AS, Jamil A, Bin FYB. Assessment of chronic ulcers using digital imaging. National postgraduate conference (NPC). 2011.p. 1-5.

(Hani 2012) Hani AFM, Arshad L, Malik AS, Jamil A, Bin FYB. Haemoglobin distribution in ulcers for healing assessment. 2012 4th international conference on intelligent and advanced systems (ICIAS), vol. 1. 2012. p. 362-7.

(He 2017) He, K.; Gkioxari, G.; Dollár, P.; Girshick, R. Mask r-cnn. In Proceedings of the IEEE International Conference on Computer Vision, Venice, Italy, 22-29 October 2017. 
(Hettiarachchi 2013) Hettiarachchi NDJ, Mahindaratne RBH, Mendis GDC, Nanayakkara

HT, Nanayakkara ND. Mobile based wound measurement. 2013 IEEE point-of-care healthcare technologies (PHT). 2013. p. 298-301.

(Hochreiter 2001) Hochreiter, S., Bengio, Y., Frasconi, P., \& Schmidhuber, J. (2001). Gradient flow in recurrent nets: the difficulty of learning long-term dependencies.

(Horn 2004) Horn SD, Bender SA, Ferguson ML, Smout RJ, Bergstrom N, Taler G, et al. The national pressure ulcer long-term care study: pressure ulcer development in longterm care residents. J Am Geriatr Soc 2004;52(3):359-67.

(Ismael 2020) Ismael, S. A. A., Mohammed, A., \& Hefny, H. (2020). An enhanced Deep Learning approach for brain cancer MRI images classification using residual networks. Artificial Intelligence in Medicine, 102, 101779.

(Jones 2000) Jones TD, Plassmann P. An active contour model for measuring the area of leg ulcers. IEEE Trans Med Imaging 2000;19(12):12021210.

(Joseph 2016) Joseph S, Panicker JR. Skin lesion analysis system for melanoma detection with an effective hair segmentation method. International conference on information science (ICIS). 2016. p. 91-6.

(Kalantari 2016) Kalantari, M.; Nechifor, M. Accuracy and utility of the Structure Sensor for collecting 3D indoor information. Geo-Spat. Inf. Sci. 2016, 19, 202-209.

(Khan 2015) Khan, S.; Paul, S.; Rao, S.S.; Krishnareddy, A. Segmenting skin ulcers based on thresholding and watershed segmentation. In Proceedings of the 
International Conference on Communications and Signal Processing (ICCSP), Melmaruvathur, India, 2-4 April 2015.

(Klonoff 2009) Klonoff DC. The increasing incidence of diabetes in the 21st century. J Diabetes Sci Technol (Online) 2009;3(1):12.

(Kolesnik 2006) Kolesnik M, Fexa A. How robust is the SVM wound segmentation? Proceedings of the 7th nordic signal processing symposium, 2006. NORSIG 2006. 2006. p. 50-3.

(Kosmopoulos 2007) Kosmopoulos D, Tzevelekou F. Automated pressure ulcer lesion diagnosis for telemedicine systems. IEEE Eng Med Biol 2007;26(5):1822.

(Le, Q.V 2015) Le, Q. V. (2015). A tutorial on Deep Learning part 2: Autoencoders, convolutional neural networks and recurrent neural networks. Google Brain, 1-20.

(Lee 2015) Lee, H.; Lee, B.U.; Park, J.; Sun, W.; Oh, B.; Yang, S. Segmentation of wounds using gradient vector flow. In Proceedings of the International Conference on Intelligent Informatics and Biomedical Sciences (ICIIBMS), Okinawa, Japan, 28-30 November 2015.

(Li 2017) Li D, Mathews C. Automated measurement of pressure injury through image processing. J Clin Nurs 2017;26(21-22):3564-75.

(Litjens 2017) Litjens, G., Kooi, T., Bejnordi, B. E., Setio, A. A. A., Ciompi, F., Ghafoorian, M., ... \& Sánchez, C. I. (2017). A survey on Deep Learning in medical image analysis. Medical image analysis, 42, 60-88.

(Lowe 2014) Lowe, D.G. Distinctive image features from scale-invariant keypoints. Int. J. Comput. Vis. 2004, 60, 91-110. 
(Lu 2017) Lu, F., Wu, F., Hu, P., Peng, Z., Kong, D., Feb. 2017. Automatic 3D liver location and segmentation via convolutional neural network and graph cut. Int J Comput Assist Radiol Surg 12, 171-182.

(Ma 2017) Ma, Y., Wang, J., Xu, H.; Zhang, S.; Mei, X.; Ma, J. Robust image feature matching via progressive sparse spatial consensus. IEEE Access 2017, 5, 24568-24579.

(Medetec) Medetec Medical Images Medetec. Available online: http://www.medetec.co.uk/files/medetec-imagedatabases.html (Mukherjee 2014) Mukherjee R, Manohar DD, Das DK, Achar A, Mitra A, Chakraborty C. Automated tissue classification framework for reproducible chronic wound assessment. Biomed Res Int 2014. Article ID 851582.

(Nandagopan 2016) Nandagopan, G.L.; Haripriya, A.B. Implementation and comparison of two image segmentation techniques on thermal foot images and detection of ulceration using asymmetry. In Proceedings of the International Conference on Communication and Signal Processing (ICCSP), Melmaruvathur, India, 6-8 April 2016.

(Noguchi 2014) Noguchi H, Kitamura A, Yoshida M, Minematsu T, Mori T, Sanada H. Clustering and classification of local image of wound blotting for assessment of pressure ulcer. Proc world automation congress (WAC) 2014:427432.

(NPUAP 2016) National Pressure Ulcer Advisory Panel. National Pressure Ulcer Advisory Panel announces a change in terminology from pressure ulcer to pressure injury and updates the stages of pressure injury. 
http://www.npuap.org/national-pressureulcer-advisory-panel-npuapannounces-a-change-in-terminology-from-pressureulcer-to-pressureinjury-and-updates-the-stages-of-pressure-injury [Last accessed 26 May 2016].

(NPUAP store) http://www.npuap.org/online-store/.

(Ortiz 2017) Ortiz DP, Sierra-Sosa D, Garcia-Zapirain B. Pressure ulcer image segmentation technique through synthetic frequencies generation and contrast variation using toroidal geometry. Biomed Eng Online 2017;16(1):4.

(Otsu 1979) Otsu N. A threshold selection method from gray-level histogram. IEEE Trans Syst Man Cybern 1979;9:62-6.

(Paluchowski 2013) Paluchowski LA, Denstedt M, Rren T, Pukstad B, Randeberg LL. A combined 3D and hyperspectral method for surface imaging of wounds. SPIE BiOS Int Soc Opt Photon 2013. pp. 85780 T85780 T-10.

(Plassmann 1998) Plassmann P, Jones T. MAVIS: a non-invasive instrument to measure area and volume of wounds. Med Eng Phys 1998;20:332338.

(Pytorch3D) PyTorch3D. Available online:

https://github.com/facebookresearch/pyTorch3d

(Raja 2014) N. Raja, V. Rajinikanth, K. Latha, Otsu based optimal multilevel image thresholding using firefly algorithm, Modell. Simul. Eng. 2014 (2014) 37. 
(Ren 2015) Ren, S., He, K., Girshick, R., \& Sun, J. (2015). Faster r-cnn: Towards real-time object detection with region proposal networks. In Advances in neural information processing systems (pp. 91-99).

(Ren 2017) Ren, S.; He, K.; Girshick, R.; Sun, J. Faster R-CNN: Towards Real-Time Object Detection with Region Proposal Networks. IEEE Trans. Pattern Anal. Mach. Intell. 2017, 39, 1137-1149.

(Rickard 2003) H.E. Rickard, G.D. Tourassi, A.S. Elmaghraby, Self-organizing maps for masking mammography images, in: Information Technology Applications in Biomedicine, 2003. 4th International IEEE EMBS Special Topic Conference on (pp. 302-305), IEEE, 2003.

(Ronneberger 2015) Ronneberger, O., Fischer, P., \& Brox, T. (2015, October). U-net: Convolutional networks for biomedical image segmentation. In International Conference on Medical image computing and computerassisted intervention (pp. 234-241). Springer, Cham.

(Seixas 2015) Seixas JL, Barbon S, Mantovani RG. Pattern recognition of lower member skin ulcers in medical images with machine learning algorithms. 2015 IEEE 28th international symposium on computer-based medical systems (CBMS). 2015. p. 50-3.

(Seixas 2015) Seixas, J.L.; Barbon, S.; Mantovani, R.G. Pattern recognition of lower member skin ulcers in medical images with machine learning algorithms. In Proceedings of the IEEE 28th International Symposium on ComputerBased Medical Systems, Sao Carlos, Brazil, 22-25 June 2015. 
(Sen, C.K. 2019) Sen, C. K. (2019). Human wounds and its burden: an updated compendium of estimates.

(Shah 2013) Shah, A.; Wollak, C.; Shah, J.B. Wound measurement techniques:

Comparing the use of ruler method, 2D imaging and 3D scanner. J. Am. Coll. Clin. Wound Spec. 2013, 5, 52-57.

(Shi 2017) Shi, J., Zheng, X., Li, Y., Zhang, Q., Ying, S., Jan. 2017. Multimodal neuroimaging feature learning with multimodal stacked deep polynomial networks for diagnosis of Alzheimer's disease. IEEE J Biomed Health Inform.

(Silveira 2009) Silveira M, Nascimento JC, Marques JS, Marçal AR, Mendonça T, Yamauchi S, et al. Comparison of segmentation methods for melanoma diagnosis in dermoscopy images. IEEE J Sel Top Signal Process 2009;3(1):35-45.

(Soldevilla 2016) Soldevilla AJ, Torra IBJ, Posnett J, Verdu SJ, San Miguel L, Mayan SM. The burden of pressure ulcers in Spain. Wounds Compend Clin Res Pract 2007;19(7):2016.

(Stages 2018) $\quad$ http://www.npuap.org/resources/educational-and-clinicalresources/npuap-pressure-injury-stage.

(Structure Sensor) Structure Sensor. 3D Scanning, Mixed Reality and more, for any Device from iPads to Robots. Available online: https://structure.io/

(Suk 2013) Suk, H. I., \& Shen, D. (2013, September). Deep Learning-based feature representation for $\mathrm{AD} / \mathrm{MCI}$ classification. In International Conference on 
Medical Image Computing and Computer-Assisted Intervention (pp. 583590). Springer, Berlin, Heidelberg.

(Suk 2015) Suk, H. I., Lee, S. W., Shen, D., \& Alzheimer's Disease Neuroimaging Initiative. (2015). Latent feature representation with stacked auto-encoder for AD/MCI diagnosis. Brain Structure and Function, 220(2), 841-859.

(Suk 2016) Suk, H. I., Wee, C. Y., Lee, S. W., \& Shen, D. (2016). State-space model with Deep Learning for functional dynamics estimation in resting-state fMRI. NeuroImage, 129, 292-307.

(Tajbakhsh 2020) Tajbakhsh, N., Jeyaseelan, L., Li, Q., Chiang, J. N., Wu, Z., \& Ding, X. (2020). Embracing imperfect datasets: A review of Deep Learning solutions for medical image segmentation. Medical Image Analysis, 101693.

(Trabelsi 2013) Trabelsi O, Tlig L, Sayadi M, Fnaiech F. Skin disease analysis and tracking based on image segmentation. 2013 international conference on electrical engineering and software applications (ICEESA). 2013. p. 1-7.

(Treuillet 2009) Treuillet S, Albouy B, Lucas Y. Three-dimensional assessment of skin wounds using a standard digital camera. IEEE Trans Med Imaging $2009 ; 28: 752762$.

(Veredas 2010) Veredas F, Mesa H, Morente L. Binary tissue classification on wound images with neural networks and Bayesian classifiers. IEEE Trans Med Imaging 2010;29:410427. 
(Veredas 2015) Veredas FJ, Luque-Baena RM, Martn-Santos FJ, Morilla-Herrera JC, Morente L. Wound image evaluation with machine learning. Neurocomputing 2015;164:112-22.

(Veredas 2015b) Veredas F, Mesa H, Morente L. Efficient detection of wound-bed and peripheral skin with statistical colour models. Med Biol Eng Comput 2015;53:345359.

(Wang 2015) Wang L, Pedersen PC, Strong DM, Tulu B, Agu E, Ignotz R. Smartphonebased wound assessment system for patients with diabetes. IEEE Trans Biomed Eng 2015;62(2):477-88.

(Wang 2016) Wang, L.; Pedersen, P.C.; Agu, E.; Strong, D.M.; Tulu, B. Area determination of diabetic foot ulcer images using a cascaded two-stage SVM-based classification. IEEE Trans. Biomed. Eng. 2016, 64, 20982109.

(Wang 2016a) Wang, C., Elazab, A., Wu, J., Hu, Q., Nov. 2016a. Lung nodule classification using deep feature fusion in chest radiography. Comput Med Imaging Graph

(Wang 2017) Wang L, Pedersen PC, Agu E, Strong DM, Tulu B. Area determination of diabetic foot ulcer images using a cascaded two-stage SVM-based classification. IEEE Trans Biomed Eng 2017;64(9):2098-109.

(Wannous 2008) Wannous H, Treuillet S, Lucas Y, Albouy B. Mapping classification results on 3D model: a solution for measuring the real areas covered by skin wound tissues. 3rd international conference on information and 
communication technologies: from theory to applications, 2008. ICTTA, 2008. 2008. p. 1-6.

(Wannous 2011) Wannous H, Lucas Y, Treuillet S. Enhanced assessment of the woundhealing process by accurate multiview tissue classification. IEEE Trans Med Imaging 2011;30:315326.

(Xu 2020) Xu, Y., Liu, Z., Li, Y., Hou, H., Cao, Y., Zhao, Y., ... \& Cui, L. (2020). Feature data processing: Making medical data fit deep neural networks. Future Generation Computer Systems.

(Yadav 2013) Yadav MK, Manohar DD, Mukherjee G, Chakraborty C. Segmentation of chronic wound areas by clustering techniques using selected color space. J Med Imaging Health Informat 2013;3(1):22-9.

(Yee 2017) Yee A, Harmon J, Yi S. Quantitative monitoring wound healing status through three-dimensional imaging on mobile platforms. J Am Coll Clin Wound Special 2017.

(Zahia 2018) Zahia, S., Sierra-Sosa, D., Garcia-Zapirain, B., \& Elmaghraby, A. (2018). Tissue classification and segmentation of pressure injuries using convolutional neural networks. Computer methods and programs in biomedicine, 159, 51-58.

(Zahia 2020b) Zahia, S.; Zapirain, M.B.G.; Sevillano, X.; González, A.; Kim, P.J.; Elmaghraby, A. Pressure injury image analysis with machine learning techniques: A systematic review on previous and possible future methods. Artif. Intell. Med. 2019, 102, 101742. 
(Zahia 2020) Zahia, S.; Garcia-Zapirain, B.; Elmaghraby, A. Integrating 3D Model Representation for an Accurate Non-Invasive Assessment of Pressure Injuries with Deep Learning. Sensors 2020, 20, 2933. 


\section{CURRICULUM VITA}

NAME: $\quad$ Sofia Zahia

ADDRESS: $\quad$ Puente de Deusto 4, Apt 8A

Bilbao, 48009, Bilbao, Spain

DOB: $\quad$ Rabat Morocco - March 6, 1995

EDUCATION

\& TRAINING: B.A., Electronics.

Enseirb-Matmeca, France, 2015

M.A., Signal and Image processing

Enseirb-Matmeca, France, 2017

Ph.D, Computer Science and Engineering

University of Louisville, USA, 2020

\section{PUBLICATIONS:}

- Zahia, S., Garcia-Zapirain, B., Saralegui, I., \& Fernandez-Ruanova, B. (2020). Dyslexia detection using 3D convolutional neural networks and functional magnetic resonance imaging. Computer Methods and Programs in Biomedicine, 197, 105726.

- $\quad$ Hameed, Z., Zahia, S., Garcia-Zapirain, B., Javier Aguirre, J., \& María Vanegas, A. (2020). Breast Cancer Histopathology Image Classification Using an Ensemble of Deep Learning Models. Sensors, 20(16), 4373.

- Zahia, S., Garcia-Zapirain, B., \& Elmaghraby, A. (2020). Integrating 3D Model Representation for an Accurate Non-Invasive Assessment of Pressure Injuries with Deep Learning. Sensors, 20(10), 2933.

- Zahia, S., Zapirain, M. B. G., Sevillano, X., González, A., Kim, P. J., \& Elmaghraby, A. (2020). Pressure injury image analysis with machine learning techniques: A systematic review on previous and possible future methods. Artificial Intelligence in Medicine, 102, 101742. 
- $\quad$ Hameed, Z., Zahia, S., Garcia-Zapirain, B., Javier Aguirre, J., \& María Vanegas, A. (2020). Breast Cancer Histopathology Image Classification Using an Ensemble of Deep Learning Models. Sensors, 20(16), 4373.

- $\quad$ Garcia-Zapirain, B., Castillo, C., Badiola, A., Zahia, S., Mendez, A., Langlois, D., ... \& Smaïli, K. (2018, September). A proposed methodology for subjective evaluation of video and text summarization. In International Conference on Multimedia and Network Information System (pp. 396-404). Springer, Cham.

- Zahia, S., Sierra-Sosa, D., Garcia-Zapirain, B., \& Elmaghraby, A. (2018). Tissue classification and segmentation of pressure injuries using convolutional neural networks. Computer methods and programs in biomedicine, 159, 51-58.

\section{AWARDS:}

- $\quad$ FPI grant awarded by the Spanish Government for a duration of 48 months (8 selected from 200 eligible applicants).

- 2nd Prize in Hack4elder competition on innovative technical solutions to help the elderly to release their full potential and added value in the society as a measure of integration in their communities.

- $\quad$ 2nd Prize in the Innovation forum Euskadi hackathon on novel technical solutions for a personalized assistance of people with Parkinson disease.

\section{RESEARCH INTEREST:}

Artificial intelligence, Machine learning, Deep Learning, Image processing, Signal processing, Computer Vision, 3D reconstruction, Data mining, Electronics.

\section{TECHNICAL SKILLS:}

Operating Systems: Mac OSX, Windows, GNU/Linux

Computer Languages: Python, $\mathrm{C} / \mathrm{C}++$, Visual Basic, Visual $\mathrm{C}++$

Scientific Applications: MATLAB/Simulink, Xilinx, Proteus (ISIS et ARES), Cadence, PSIM

Technical Drawing: Blender, AutoCAD, VXelements, VXModel 
Office Applications: Microsoft PowerPoint, Access, Excel, Word, Lotus Notes.

Internet Development: Django, MSQ 